\title{
MECHANISMS OF PHOTORECEPTOR MEDIATED RESPONSES IN PHOTOMORPHOGENESIS AND PHOTOTROPISM
}

\author{
A Dissertation \\ presented to \\ the Faculty of the Graduate School \\ at the University of Missouri-Columbia \\ In Partial Fulfillment \\ of the Requirements for the Degree \\ Doctor of Philosophy \\ by \\ KYLE WILLENBURG \\ Dr. Emmanuel Liscum, Dissertation Supervisor \\ MAY 2018
}


The undersigned, appointed by the dean of the Graduate School, have examined the dissertation entitled

"MECHANISMS OF PHOTORECEPTOR MEDIATED RESPONSES IN PHOTOMORPHOGENESIS AND PHOTOTROPISM"

presented by Kyle Willenburg,

a candidate for the degree of doctor of philosophy, biology

and hereby certify that, in their opinion, it is worthy of acceptance.

Professor Emmanuel Liscum, III

Professor Kathleen Newton

Professor David Braun

Professor David Mendoza-Cózatl 


\section{DEDICATIONS}

I would like to dedicate this work to my wife Ellie. I want to say thank you for your support throughout the process of my research and during the writing of this manuscript. Your encouragement and friendship have provided me with the energy and focus to persevere through the long, difficult weeks of research and writing. You have sacrificed much for my sake during my degree completion and done so freely and generously.

I also want to say thank you to my daughter Talia. Though she is not yet two, she has brought me joy and happiness at the end of long days and given me more inspiration towards diligence and hard work than I ever thought was possible. I hope never to bear the cost of your smiles and laughter again.

I want to thank my parents and siblings for their support over the course of my education, for the encouragement to pursue my life passion of science. Thank you for your patience with me as I grew up. Sorry Mom for using all your dishes to do science experiments in. Sorry Kenny for growing fruit flies, black-widow spiders and the hundred other things in our shared bedroom. Thanks Jessica for thinking I was cool when I was rambling about some new thing I learned. Thank you Dad for teaching me what it means to work and sacrifice for your family. 


\section{ACKNOWLEDGEMENTS}

I would like to thank Dr. Mannie Liscum for guidance during my degree. His mentorship has created an environment where scientific development was beneficially challenging and enjoyable, where I was encouraged and helped to develop as a scientist. His leadership has greatly impacted my abilities to present science in written and oral formats to scientific and lay audiences, think critically about science broadly—especially my own research—and accept ownership for my research.

I would like to thank each member of my committee: Mannie Liscum, David Braun, Kathy Newton, and David Mendoza for the critical evaluation of my research and guidance over the years. Your hard advice has truly been a great benefit to me and my research. Thank you for your keen insight and critical evaluations over the years.

I would also like to acknowledge past and current lab members who have additionally offered insight into my research and lab methods. I would like to thank Dustin Mayfield-Jones, Diana Robert-Coats, Preston Owen, Anthony Schumate, Dan Leuchtman, Scott Askinosie, Brianna Heerhold, Brittany CruzanJohnson, Krista Shucart, Katelynn Koskie, Patrick Nittler, and Johanna Morrow for advice, proofreading, critical insights, camaraderie, laughs, and friendship. You shall ever be remembered with happy memories. 


\section{TABLE OF CONTENTS}

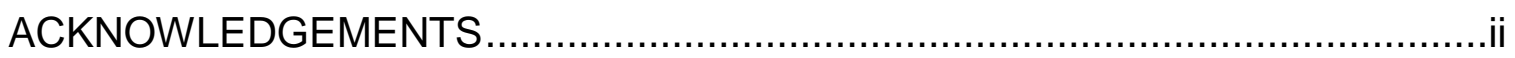

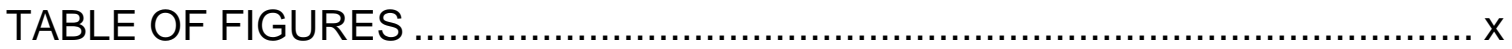

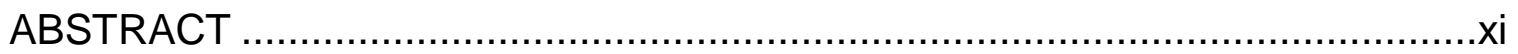

Chapter 1 LITERATURE REVIEW …..................................................... 1

phototropin 1 and 2 are blue light photoreceptors ................................... 1

The phots undergo a conformational change upon blue light perception that

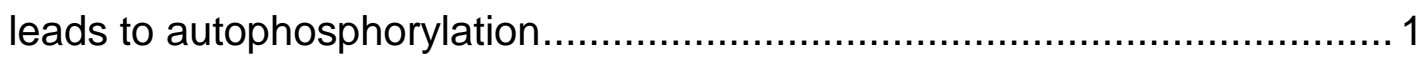

Autophosphorylation of phot1 leads to protein internalization ...................... 3

Functional Relevance of phototropin internalization ................................. 4

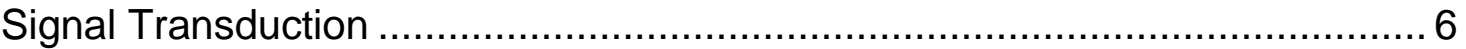

NONPHOTOTROPIC HYPOCOTYL3 (NPH3), a phot-interacting protein, is

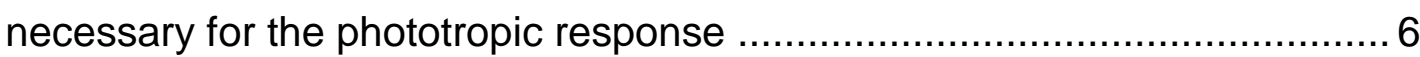

$\mathrm{NPH} 3$ is a phosphoprotein in dark and is rapidly dephosphorylated upon

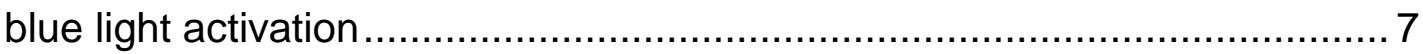

A role for $\mathrm{NPH} 3$ as a substrate adaptor in an E3 ubiquitin ligase complex.... 8 NPH3, along with ROOTPHOTOTROPISM2 (RPT2), are founding members of the NPH3-RPT2-Like (NRL) 33-member protein family ......................... 9

While the general structures of the NRL proteins are similar, their functions

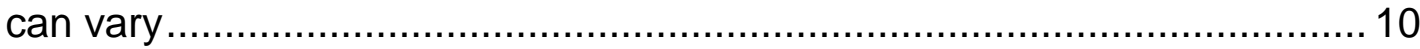


PHYTOCHROME KINASE SUBSTRATE (PKS) proteins are important for the phototropic response and may shed light on the involvement of the redlight photoreceptor phytochrome in this response

Cytosolic calcium is a possible intermediary signal within the phototropic pathway

ENHANCED BENDING1 (EHB1), and ADP-RIBOSYLATION FACTOR GTPase-ACTIVATING PROTEIN (ADG12) are NPH3-interacting proteins shown to be involved in the phototropic response

A potential role for Heterotrimeric $\mathrm{G}$ proteins in phototropism

Protein phosphatases have a variety of functions within phototropic signal transduction

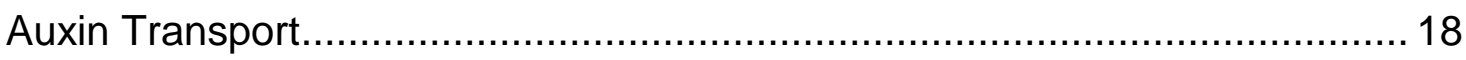

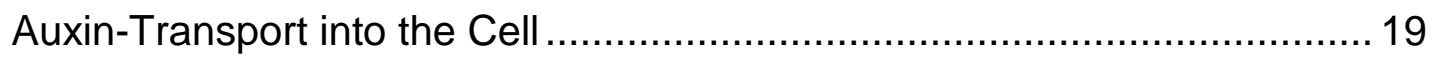

Auxin efflux is facilitated in concert through proteins in two different gene families

Regulation of PIN proteins involves intracellular cycling, phosphorylation and members from the AGC protein kinase family 21

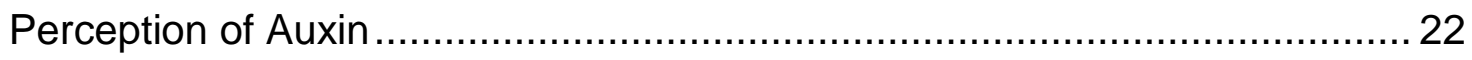

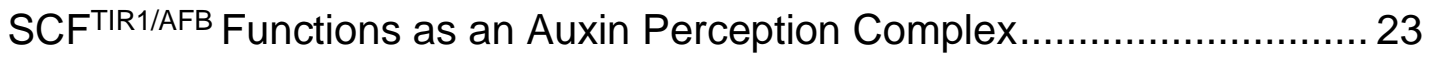

SCF Complex Proteins: Who they are and what they do ……................... 24

Auxin Signaling: Linking Auxin Perception to Activation ............................ 24 
The Mechanisms by which the SCF ${ }^{\text {TIR1/AFB }}$ Complex Senses Auxin and

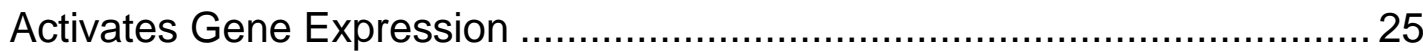

Transcriptional Modification Induced by the Polar Auxin Gradient Leads to

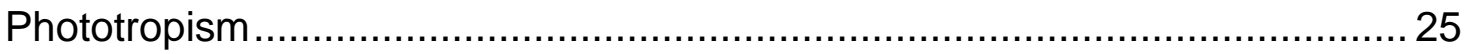

Auxin Triggered Tropic Genes....................................................... 26

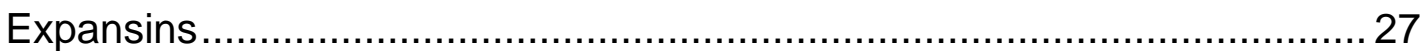

Auxin Conjugating Enzymes GH3.5/WES1 and GH3.6/DFL1 May Attenuate Auxin Induced Transcription During Tropism.......................................... 29

SMALL AUXIN UP RNAs (SAURs) and Tropisms.................................... 30

HATs and Auxin-Induced Hypocotyl Elongation ....................................... 31

SKS1 is a Glycosylphosphatidylinositol-Anchored (GPI-Anchored) Protein 32 A bHLH May Serve as an Additional Regulator of Tropic Induced Hypocotyl Elongation 32

Other Modifiers of Phototropism 34

Phytochrome A: Phytochromes are Red/Far-Red Reversible Light Receptors 34

Cryptochromes 38

Katanin and Microtubule Reorientation is Necessary for Phototropism 39 Chapter 2 DECREASE IN PHOTOTROPIC CURVATURE RESULTANT OF SITE-DIRECTED MUTAGENESIS IN PHYTOCHROME A UBIQUITINATED LYSINE RESIDUES 


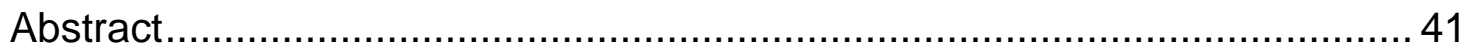

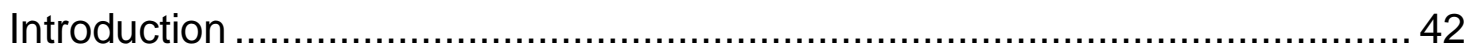

Plants use photoreceptors to sense light and modulate growth ................. 42

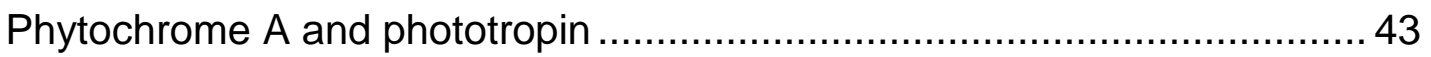

phot1 is a blue light photoreceptor that requires the E3 ubiquitin ligase

substrate adapter $\mathrm{NPH} 3$, an NRL family member, for phototropic signaling 44

Type 1 phytochromes are ubiquitinated and rapidly degraded ................... 45

Ubiquitin remnant profiling screen revealed phyA exhibits a CRL3 ${ }^{\mathrm{NPH} 3}{ }_{-}$

dependent ubiquitination in blue light ................................................... 46

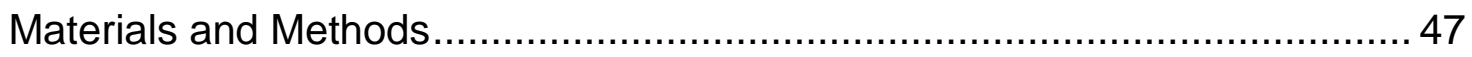

Plant Growth and Treatment Conditions................................................ 47

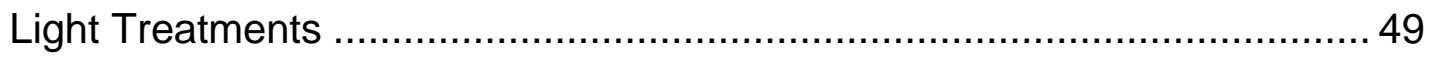

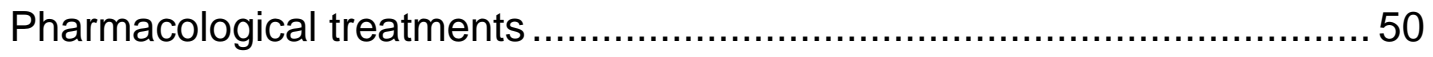

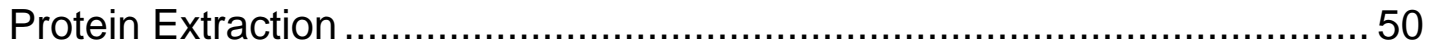

Running SDS-Polyacrylamide Gels and immunoblotting ..........................51

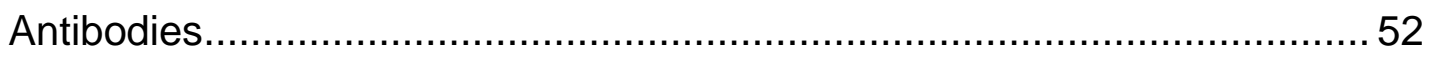

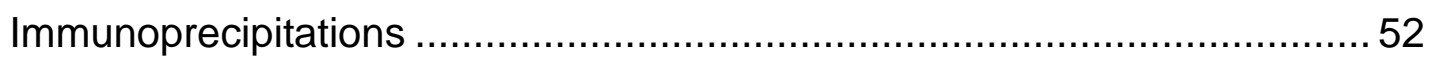

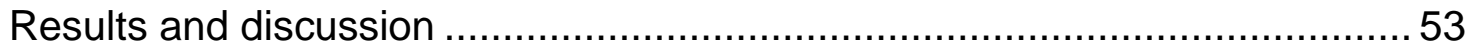

Transgenic lines expressing phy $\mathrm{A}^{\mathrm{K} 555 \mathrm{R}, \mathrm{K} 603 \mathrm{R}}$-GFP exhibit normal red lightinduced phyA degradation and far-red light-induced growth inhibition .........53 
phyA ${ }^{\mathrm{K} 555 R, \mathrm{~K} 603 \mathrm{R}}$ lines exhibit normal phototropism in low and very low fluence blue light 56

phy $A^{K 555 R,}$, K603R_GFP lines do not exhibit red light-induced phototropic enhancement.

phy $A^{K 555 R, K 603 R}$ lines have reduced phototropic curvature in high intensity blue light 60

Altered high intensity blue light-induced phototropism in phyA ${ }^{\mathrm{K} 555 R, \mathrm{~K} 603 \mathrm{R}}$ lines does not result from altered growth rates 62 phy $A^{K 555 R, K 603 R}$ lines have reduced phyA turnover in high intensity blue light

Overexpression of oat phyA in A. thaliana does not phenocopy the phy $A^{\mathrm{K} 555 \mathrm{R}, \mathrm{K} 603 \mathrm{R}}$ lines for phototropism in high intensity blue light 69 phot1, phot2, nor NPH3 appear to influence the turnover of phyA in high intensity blue light

Chapter 3 COMPARISON OF LIGHT-REGULATED DEVELOPMENT DURING SEEDLING ESTABLISHMENT IN GLYCINE MAX AND GLYCINE SOJA.

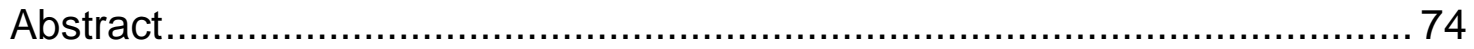

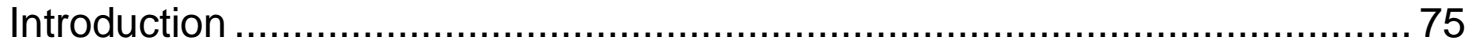

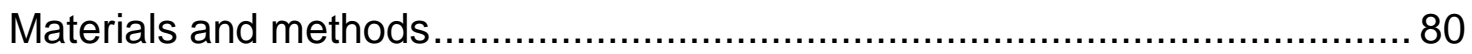

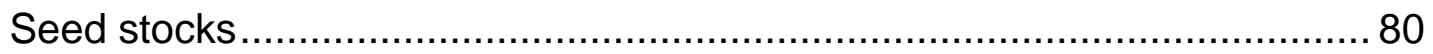

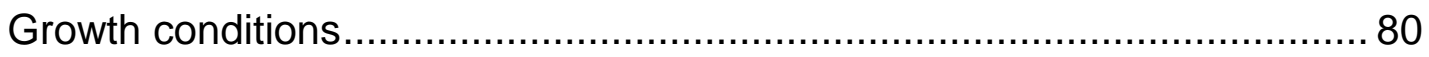


Light sources

Results

Responses of hypocotyls and first internode to white light 84

Responses of hypocotyls and first internode to blue light......................... 87

Response of hypocotyl and first internode to red light .............................. 89

Responses of hypocotyl and first internode to far-red light........................ 92

Development of second internode under different light qualities ................ 95

Root elongation in response to different light conditions ........................... 97

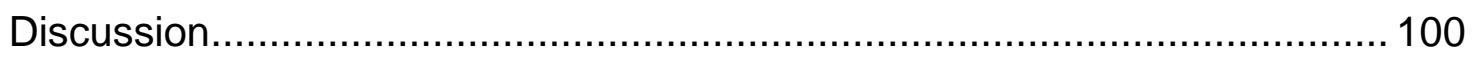

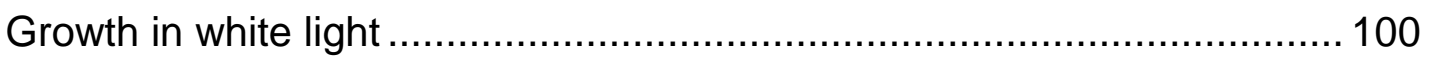

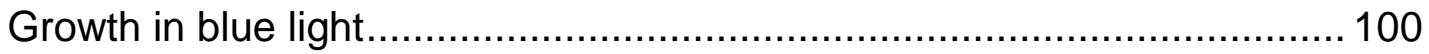

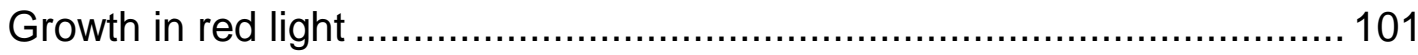

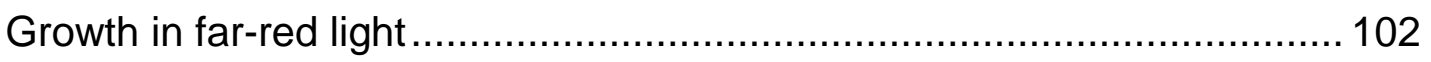

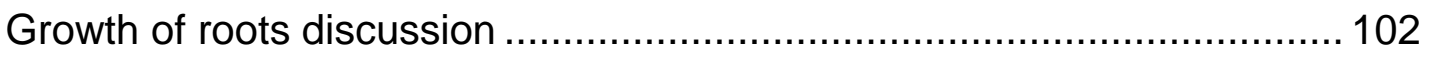

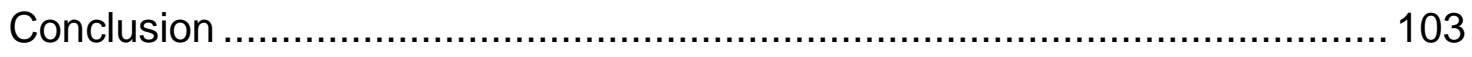

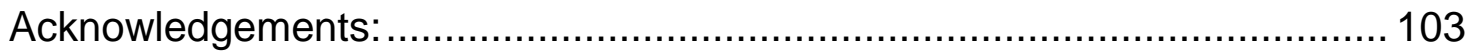

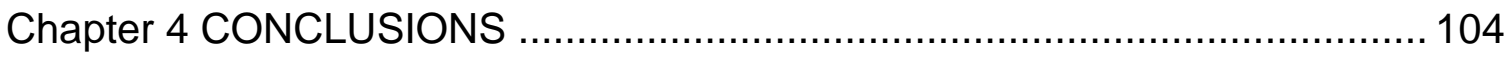

phy $\mathrm{A}^{\mathrm{K} 555 \mathrm{R}, \mathrm{K603R}}$ lines are degraded in red light and exhibit far-red lightinduced hypocotyl growth inhibition ........................................................ 105 
phy $A^{K 555 R, K 603 R}$ lines display normal blue light-induced phototropism under low and very low intensity conditions 105 phy $A^{K 555 R, ~ K 603 R ~ l i n e s ~ d i s p l a y ~ r e d u c e d ~ p h o t o t r o p i c ~ b e n d i n g ~ i n ~ h i g h ~ i n t e n s i t y ~}$ blue light 106 phy $A^{K 555 R, K 603 R}$ is stabilized in high intensity blue light 106 phy $A^{K 555 R, K 603 R}$ ubiquitination levels are not detectably less than in the phyAGFP transgenic control in immunoblots of immunoprecipitated proteins ... 106 phy $\mathrm{A}^{\mathrm{K} 555 \mathrm{R}, \mathrm{K} 603 \mathrm{R}}$ degradation is not controlled by phot1, phot2, or NPH3 under high intensity blue light conditions 107

Glycine max and Glycine soja display widely different growth responses when grown in far-red light 107 Glycine max and Glycine soja display a generally similar pattern of growth in white, blue, and red light. 108 Glycine max produces a second internode more frequently in all light conditions than Glycine soja 108

Glycine max produces robust root growth in all light conditions and in darkness while G. soja produces root growth when stimulated by light..... 108 LITERATURE CITED: 110

VITA 158 


\section{TABLE OF FIGURES}

Figure 2.1 Red Light-induced degradation of phyA is not altered in phy ${ }^{k 555 R, ~ K 603 R ~ l i n e s . ~}$

Figure 2.2 Far-red light-induced hypocotyl grownth inhibition in phyA ${ }^{\mathrm{K} 555 R \text {, }}$ $\mathrm{K} 603 \mathrm{R}$ lines is not different from phyA-GFP or Col. 55

Figure 2.3 phy ${ }^{\mathrm{K} 555 \mathrm{R}, \mathrm{K} 603 \mathrm{R}}$ lines respond phototropically normal to low and very-low fluence rate blue light.

Figure 2.4 phy $\mathrm{A}^{\mathrm{K} 555 \mathrm{R}, \mathrm{K} 603 \mathrm{R}}$ lines did not exhibit red light-enhancement of phototropic curvature in low fluence blue light.

Figure 2.5 phy $\mathrm{A}^{\mathrm{K} 555 \mathrm{R}, \mathrm{K} 603 \mathrm{R}}$ lines responded prototropically at reduced levels to high intensity blue light-treatments compared to Col-0, phyA-GFP, and phyA-211

Figure 2.6 phy $\mathrm{A}^{\mathrm{K5} 55 \mathrm{R}, \mathrm{K} 603 \mathrm{R}}$ lines are functional in hypocotyl elongation ...... 63

Figure 2.7 phy $A^{\mathrm{K} 555 \mathrm{R}, \mathrm{K603R}}$ lines display reduced phyA degradation in high blue light.

Figure 2.8 Transgenic A. thaliana lines over-expressing oat phyA do not exhibit altered phototropism in response to high intensity blue light .........70

Figure 2.9 phot1, phot2, nor NPH3 modulate phyA degradation in high fluence blue light

Figure 3.1 Drawing of two-week-old G. max and G. soja seedlings ............ 83

Figure 3.2 White light growth for G. max and G. soja ........................... 86

Figure 3.3 Blue light growth is inhibited in the hypocotyl but induced in the second internode.

Figure 3.4 Red light growth is inhibited in the hypocotyl but induced in the first internode

Figure 3.5 Far- red light growth is inhibited in the hypocotyl but induced in the second internode for G. max but not for G. soja .

Figure 3.6 Percent of seedlings producing a second internode in various light qualities

Figure 3.7Root length in G. max is stimulated in white, blue, and far-red light but not stimulated in red light, whereas G. soja is stimulated in all light conditions and more responsive to light that $G$. max 


\section{ABSTRACT \\ MECHANISMS OF PHOTORECEPTOR MEDIATED RESPONSES IN \\ PHOTOMORPHOGENESIS AND PHOTOTROPISM \\ Kyle Trent Willenburg \\ Dr. Emmanuel Liscum, III, Dissertation Supervisor}

Photoreceptor facilitated light perception provides plants with critical

environmental information from which they can direct their growth. We

investigated the early photomorphogenic responses in Glycine max and Glycine

soja with the aim of elucidating the effect that domestication has had on early

light responses. Our study indicates that $G$. max exhibits growth in darkness that is more characteristic of growth in light. This finding suggests that domestication has resulted in a crop species that is partially constitutively photomorphogenic. We additionally investigated the effects of blue light-induced ubiquitination sites that were ubiquitinated dependently by NON-PHOTOTROPIC HYPOCOTYL3 (NPH3) in the red light photoreceptor phytochrome A (phyA) in Arabidopsis thaliana. We discovered that transgenic lines expressing phyA with the ubiquitination sites K555 and K603 converted to non-ubiquitinatable arginine residues displayed reduced degradation in blue light and additionally reduced phototropic curvature in high intensity blue light, while functioning normally in other responses tested: very low and low intensity blue light phototropism, far-red light hypocotyl growth inhibition, and red light-induced phyA degradation. This finding suggests a mechanism for a more direct route of blue and red lightsignaling crosstalk than has been previously shown. 


\section{Chapter 1 LITERATURE REVIEW}

by Johanna Morrow and Kyle Willenburg

Sections below by Johanna Morrow

\section{phototropin 1 and 2 are blue light photoreceptors}

The PHOTOTROPIN family in Arabidopsis thaliana contains two members:

PHOT1 and PHOT2, and these two genes are present in all higher plants (Briggs \& Huala, 1999), while there has been duplication of these genes in some species (F.-W. Li et al., 2015). In the context of phototropic function, phot1 has been shown to be the primary photoreceptor under low fluence light, while both phot1 and phot2 act redundantly under moderate to high fluence conditions (Khurana \& Poff, 1989; E. Liscum, 1995; Sakai et al., 2001; Sakai, Wada, Ishiguro, \& Okada, 2000). In addition to the phototropic pathway, the phots act redundantly in a variety of other functions that impact optimization of photosynthesis. These functions include: stomatal opening, chloroplast accumulation/avoidance, cotyledon/leaf movement, expansion and leaf flattening (I. S. Han, Cho, Moni, Lee, \& Briggs, 2013; S. -i. Inoue et al., 2008; Kinoshita, Doi, \& Suetsugu, 2001; Ohgishi, Saji, Okada, \& Sakai, 2004; Sakai et al., 2001; Sakamoto, 2002; Takemiya, Inoue, \& Doi, 2005); (Jarillo et al., 2001; Kagawa et al., 2001; Sakai et al., 2001).

\section{The phots undergo a conformational change upon blue light perception that leads to autophosphorylation}


phots contain an amino-terminal sensory region and carboxyl-terminal output region (John M. Christie et al., 1998). The sensory region contains two LOV (light, oxygen, and voltage) domains, which in darkness each noncovalently binds a Flavin mononucleotide (FMN) chromophore (Huala et al., 1997), while the output region encodes a serine/threonine protein kinase domain (PKD) which is a member of the AGC protein kinase family (Bögre, Ökrész, Henriques, \& Anthony, 2003; Huala et al., 1997). The LOV domains represent a subset of the Per-Arnt-Sim (PAS) domains that are present in a variety of proteins with diverse functions across a large number of organisms (Rojas-Pirela et al., 2018). The LOV domains were named because the function of the proteins in which they are found are controlled by light, oxygen, and/or voltage (Crosson, Rajagopal, \& Moffat, 2003; Huala et al., 1997). The LOV domains within phototropin have each been shown to have a distinct function: LOV1 is involved in protein dimerization, while LOV2 functions as a molecular light activated switch for repression/derepression of the PKD (S. M. Harper, Christie, \& Gardner, 2004; Jones, Feeney, Kelly, \& Christie, 2007; Nakasako, Zikihara, Matsuoka, Katsura, \& Tokutomi, 2008; Michael Salomon, Lempert, \& Rüdiger, 2004; Sullivan, Thomson, Lamont, Jones, \& Christie, 2008; Tokutomi, Matsuoka, \& Zikihara, 2008). Upon blue light absorption, the FMN chromophore becomes covalently attached to a cysteine residue within each LOV domain, forming an adduct that leads to a progressive conformational change in the protein that moves from the chromophore-binding pocket out to an alpha-helix (the Ja-helix, located in the linker region between LOV2 and PKD). In darkness the protein conformation 
keeps the PKD in a repressed/inactive state. Blue light-activation leading to the aforementioned conformational changes leads to de-repression of the PKD and

allows for protein autophosphorylation/transphosphorylation of the receptor (J. M. Christie, Salomon, Nozue, Wada, \& Briggs, 1999; S. -i. Inoue et al., 2008; M.

Salomon, Christie, Knieb, Lempert, \& Briggs, 2000; Sullivan et al., 2008). This autophosphorylation is critical for the phototropic response (S. -i. Inoue et al., 2008; Tseng \& Briggs, 2010). Within phot1 two serine residues (position S849\&S851) have been identified as critical sites for this auto/transphosphorylation; serine851 is critical for phototropism (S. -i. Inoue et al., 2008). Within phot2 the phosphorylated serine residues (position S761\&S763) have been shown to be important, but a loss of phosphorylation at these sites does not lead to a complete loss of function as it does in phot1, indicating that there are likely additional phosphorylation site(s) within phot2 that are necessary for the phototropic response (S. -i. Inoue et al., 2011).

\section{Autophosphorylation of phot1 leads to protein internalization}

Though no transmembrane domain is present, in darkness the phots are associated with the plasma membrane; additionally, the carboxyl-terminal portion of the PKD is required for membrane association (Kong et al., 2007; Kong, Suetsugu, et al., 2013; Kong, Kagawa, Wada, \& Nagatani, 2013). However, upon blue light activation, a portion of the pool of phot protein becomes internalized (I.S. Han, Tseng, Eisinger, \& Briggs, 2008; Kong et al., 2006; Sakamoto, 2002; Y. L. Wan et al., 2008). It has been shown that this internalization is dependent upon auto/transphosphorylation in the case of phot1 (Kaiserli, Sullivan, Jones, 
Feeney, \& Christie, 2009; Sullivan, Kaiserli, Tseng, \& Christie, 2010). A mutation within phot1 (I608E) leading to a structural change allowing de-repression of the kinase domain renders the protein constitutively active and results in protein internalization in the absence of light. Conversely, no internalization is observed phot1 mutant that is phosphorylation-incompetent (D806N), indicating the necessity of auto/transphosphorylation for protein internalization (S. M. Harper et al., 2004; Kaiserli et al., 2009). Interestingly, similar to phot1, internalization of phot2 requires the carboxyl-terminal PKD, but phot2 internalization does not require auto/transphosphorylation of the protein; blue light activation alone is sufficient (Aggarwal et al., 2014; Kong et al., 2006).

\section{Functional Relevance of phototropin internalization}

Protein internalization, in general, has been shown to be involved in the endocytic pathway (Doherty \& McMahon, 2009). Indeed, both phots have been shown to interact with clathrin (a coat protein involved in membrane trafficking); phot1 interacts with clathrin heavy chain and phot2 interacts with clathrin light chain (Kaiserli et al., 2009; Kong et al., 2006; Roberts et al., 2011). While it still has not been identified where in the cell phot1 relocates upon internalization, phot2 has been shown to move from the plasma membrane/cytoplasm to the Golgi (Aggarwal et al., 2014; Kong et al., 2006). Interestingly, it has been shown that new microtubules within the hypocotyl are generated in response to blue light stimulation and that this generation of new microtubules is phot-dependent (Lindeboom et al., 2013). Interestingly, plants deficient in Katanin1, a protein that severs microtubules leading to the further growth of the microtubule, have been 
shown to exhibit reduced phototropic bending (Lindeboom et al., 2013). How this may or may not connect to phot internalization remains unknown.

Though phots can move from the plasma membrane, it is important to ask where phot signaling occurs: the plasma membrane, the cytoplasm, or another compartment/location. It has been shown that seedlings pretreated with red light prior to phototropic blue light stimulation exhibit enhanced responses that are more rapid than in seedlings treated only with blue light (A. Janoudi \& Poff, 1993; a K. Janoudi, Gordon, Wagner, Quail, \& Poff, 1997; Janoudi A-K, Konjevic, Apel, \& Poff, 1992). Additionally, phot1 is retained at the plasma membrane in seedlings pretreated with red light, suggesting that phot1 signals from the plasma membrane (I.-S. Han et al., 2008). More support for plasma membrane associated phot representing the "functional" receptor comes from a study in which phot 1 is irreversibly anchored to the plasma membrane via a myristylation/farnestylation tag (Preuten, Blackwood, Christie, \& Fankhauser, 2015). Seedlings carrying irreversibly-anchored phot1 exhibit a normal bending response; internalization may not be necessary for primary signaling, but it could be involved in receptor turnover or recycling (Aggarwal et al., 2014; Kong et al., 2006; Emmanuel Liscum, 2016; Preuten et al., 2015). Indeed, both phots have been shown to be degraded by the 26S proteasome (Aggarwal et al., 2014; Roberts et al., 2011). 


\section{Signal Transduction}

Though the signal transduction pathway from activated phototropin to phototropic curvature has not been reconstructed in its entirety, several components of the pathway(s) have been identified and studied in detail.

\section{NONPHOTOTROPIC ㅂYPOCOTYL3 (NPH3), a phot-interacting}

\section{protein, is necessary for the phototropic response}

$\mathrm{NPH} 3$ was identified in a screen for non-phototropic mutants and has been shown to be critical for the phototropic bending response (E. Liscum, 1995; E Liscum \& Briggs, 1996; Motchoulski \& Liscum, 1999; Okadaa \& Shimuraab, 1992). The NPH3 protein is characterized by an amino-terminal BTB (broad complex, tamtrack, and bric a brac) domain (Stogios, Downs, Jauhal, Nandra, \& Privé, 2005), a central NPH3 domain (Pfam, PF03000), and a carboxyl-terminal coiled coil (CC) domain (Motchoulski \& Liscum, 1999). The CC domain of NPH3 has been shown to facilitate interaction with phot1 (Motchoulski \& Liscum, 1999) as well as phot2 (de Carbonnel et al., 2010). In addition to its role in photdependent phototropism, NPH3 has been shown to be involved in phot-mediated leaf flattening and leaf movement, but has no role in chloroplast movement or stomata opening (de Carbonnel et al., 2010; Inada, Ohgishi, Mayama, Okada, \& Sakai, 2004; S. I. Inoue, Kinoshita, Takemiya, Doi, \& Shimazaki, 2008). 


\section{$\mathrm{NPH} 3$ is a phosphoprotein in dark and is rapidly}

\section{dephosphorylated upon blue light activation}

Motchoulski and Liscum (1999) identified an enhanced mobility shift of the NPH3 protein based on light condition on an SDS-PAGE gel. It was subsequently shown that this light-dependent mobility shift results from dephosphorylation, as this modification could be recreated in the absence of light treatment by application of lambda phosphatase; additionally, treatment with a phosphatase inhibitor blocked the modification induced by blue light (Pedmale \& Liscum, 2007). Furthermore, by using pharmacological inhibitors, it was demonstrated that blocking NPH3 dephosphorylation also abrogates phototropic curvature (Pedmale \& Liscum, 2007). The dephosphorylation of NPH3, which appears to occur as the result of a yet unidentified type 1 protein phosphatase, has also been shown to be dependent upon the presence of phot1 (Pedmale \& Liscum, 2007). The aforementioned observations suggest the phototropically active signaling state of $\mathrm{NPH} 3$ is the dephosphorylated form.

An in-silica phosphorylation site prediction study was performed to identify potential phosphorylation sites within NPH3 implicating 21 sites as phospho-sites (Tsuchida-Mayama et al., 2008). Each of these subsequent predicted phosphorylation sites (serine, threonine, or tyrosine) were mutated to an alanine to render the site phosphorylation incompetent, and the mutants were examined for their phototropic responsiveness. No change in the phototropic nature of plants expressing these mutated phosphorylation sites was observed (Tsuchida- 
Mayama et al., 2008), which lends further support to the hypothesis that the dephosphorylated form of $\mathrm{NPH} 3$ is the active form of the protein in the phototropic pathway.

\section{A role for NPH3 as a substrate adaptor in an E3 ubiquitin ligase complex}

Previous studies have shown BTB domain-containing proteins can interact with Cullin3 (CUL3) proteins (Genschik, Sumara, \& Lechner, 2013). A. thaliana contains two CUL3 proteins: CUL3A and CUL3B, which are functionally redundant, and a loss-of-function of both proteins results in lethality (Figueroa, P., et al., 2005). However, a cullin3 hypomorph (cul/3yp) was developed as a tool to use in forward genetics studies, which results from a knockout of CUL3B and a knockdown of CUL3A (Thomann et al., 2009). Roberts and colleagues (2011) utilized cul3 $3^{\text {hyp }}$ to ask if CUL3 is involved in the phototropic pathway; the results showed a significant decrease in phototropic curvature while retaining its gravitropic function. These findings indicate that CUL3 proteins are not only involved in the phototropic pathway, but that they likely function early in the pathway because mutations did not impact differential growth generally (Roberts et al., 2011). The authors further showed CUL3 co-localizes and interacts with $\mathrm{NPH} 3$ at the plasma membrane.

CUL3 constitutes one component of a Cullin Ring Ligase (CRL) complex, a class of E3 ubiquitin ligase complexes that ubiquitinates proteins (Deshaies \& Joazeiro, 2009; Haglund \& Dikic, 2005; Hotton \& Callis, 2008). Roberts and 
colleagues (2011) were able to show that phot1 is ubiquitinated and this ubiquitination occurs in a NPH3- and blue light-dependent fashion. Given that $\mathrm{NPH} 3$ interacts with CUL3, the authors concluded that NPH3 is functioning as a substrate adapter in a CRL3 complex that targets phot1 for ubiquitination (Roberts et al., 2011). Interestingly, a single ubiquitin or a chain of polyubiquitin molecules may be transferred to proteins in the ubiquitination process, and phot1 exhibits variation in its ubiquitination type depending on the blue light intensity. Phot1 is poly-ubiquitinated in high fluence rate conditions $\left(>1 \mu \mathrm{mol} \mathrm{m} \mathrm{m}^{-2} \mathrm{~s}^{-1}\right)$, stimulating its degradation by the $26 \mathrm{~S}$ proteasome, while phot 1 is mono/multiubiquitinated under low fluence light $\left(<1 \mu \mathrm{mol} \mathrm{m}^{-2} \mathrm{~s}^{-1}\right)$ (Roberts et al., 2011). Recent data suggests that the purpose of mono/multiubiquitination is to keep phot1 at the plasma membrane as a signaling competent receptor (Askinosie \& Liscum, 2016).

\section{NPH3, along with ㅁOTPHOTOIROPISM2 (RPT2), are founding members of the NPH3-RPT2-Like (NRL) 33-member protein} family

As previously mentioned, NPH3 is characterized by an amino-terminal BTB domain, a carboxyl-terminal coiled coil domain, and a central conserved sequence-specific region referred to as the NPH3 domain. The NRL proteins all contain the NPH3 central region; however, some are missing the carboxylterminal coiled coil domain, and two do not contain the amino-terminal BTB domain (Pedmale, Celaya, \& Liscum, 2002). An NPH3 ortholog named 
COLEOPTILE PHOTOTROPISM1 (CPT1) has been identified by mutation in rice (K. Haga, 2005). Similar to NPH3, a loss of CPT1 function results in a nonphototropic phenotype but retains a normal gravitropic response. Also like NPH3, CPT1 is composed of an amino-terminal BTB domain, a central NPH3 domain, and a carboxyl-terminal CC domain (K. Haga, 2005). While all land plants contain one or more NRL gene, a fewer are found within earlier species (J. Christie, Suetsugu, Sullivan, \& Wada, 2017; Suetsugu et al., 2016). Interestingly, Li and colleagues (2011) observed that NPH3/NRL function often seems to involve a member of the AGC Kinase Family and/or the plant hormone auxin (as will be noted in subsequent sections).

\section{While the general structures of the NRL proteins are similar, their functions can vary}

RPT2 is the only NRL member that has been shown to play a role in phototropism (Sakai et al., 2000). In high light conditions where RPT2 function is obvious in the phototropic pathway, it has been shown that response is dependent on the phosphorylation state as well as the localization of NPH3 (Ken Haga, Tsuchida-Mayama, Yamada, \& Sakai, 2015; Sakai et al., 2000). RPT2 also interacts with phot1 and has been shown to be involved in leaf flattening, stomatal opening, and chloroplast accumulation responses (Akiko Harada, Takemiya, Inoue, Sakai, \& Shimazaki, 2013; Inada et al., 2004; Kozuka,

Suetsugu, Wada, \& Nagatani, 2013; Suetsugu et al., 2016). 
Recently, another NRL member, NRL31/NCH1 (NRL PROTEIN FOR CHLOROPLAST MOVEMENT 1) was shown to be involved in phot-mediated chloroplast accumulation (Suetsugu et al., 2016). Quite independently, NRL31 was identified as SR1IP1 (AtSR1 interaction protein 1) and shown to play a role in plant immunity (Zhang, Du, Shen, Yang, \& Poovaiah, 2014). Interestingly, NRL31/NCH1/SR1IP1 was shown to interact with CUL3A through its BTB domain and act as a substrate adapter in an E3 ubiquitin ligase complex involved in ubiquitination of AtSR1 in the pathogenesis response (Zhang et al., 2014). Suetsugu and colleagues (2016) also demonstrated that NRL31/NCH1/SR1IP1 interacts with phot1 through its $\mathrm{CC}$ domain, much as NPH3 does (Motchoulski \& Liscum, 1999).

NRL20 has been characterized to function in organ development and renamed NAKED PINS IN YUC1 (NPY1)/ENHANCER OF PINOID (ENP1)/MACCHI-BOU4 (MAB4) to reflect mutant phenotypes (Cheng, Qin, Dai, \& Zhao, 2007; Furutani et al., 2007; Treml, et al., 2005). Four other NRL genes, NRL 6, 7, 21, and 30 together with NRL 20 form a sub-clade of the NRL family (Cheng, Qin, Dai, \& Zhao, 2008). Each of these genes have been shown to have overlapping expression patterns, and NRL20/NPY1/ENP1/MAB4, NRL7/NPY3, and NRL30/NPY5 have been implicated in organogenesis (Cheng et al., 2008). Interestingly, AGC kinases PINOID (PID), PID2, WAG1 and WAG2, which are in the same subfamily as phot 1 and phot2, have been shown to be involved in NRL/NPY-regulated organogenesis (S. R. M. Bennett, Alvarez, Bossinger, \& Smyth, 1995; Cheng et al., 2008; Christensen, Dagenais, Chory, \& Weigel, 
2000). Each of these AGC kinases appears to regulate auxin responsiveness via NRL/NPY function (Cheng et al., 2008). More recently, the NPY's have been shown to be involved in yet another response mediated by auxin: root gravitropism (Yuanting Li, Dai, Cheng, \& Zhao, 2011).

Two additional NRL members have been shown to regulate functions linked to auxin. Namely, NRL23/ㅁEFECTIVELY ORGANIZED IRIBUTARIES3 (DOT3), was identified in a mutant screen for seedlings defective in vein patterning (Petricka, Clay, \& Nelson, 2008). Auxin has been shown to be a key regulator in vascular development (Y. Zhao, 2010). NRL8/SETH6 was also identified in a mutant screen and shown to be involved in pollen germination (Lalanne et al., 2004). Previous studies have shown that auxin plays a role in pollen development (Zhang, X. S., \& O'Neill, 1993), which again links the NRL protein function to auxin signaling pathways, that may or may not involve AGC Kinases (Cheng et al., 2008; Rademacher \& Offringa, 2012).

PHYTOCHROME KINASE SUBSTRATE (PKS) proteins are important for the phototropic response and may shed light on the involvement of the red-light photoreceptor phytochrome in this response

PKS1 was initially identified as a phytochrome-interacting protein that can interact with both the red $\left(\mathrm{P}_{\mathrm{r}}\right)$ and far-red absorbing $\left(\mathrm{P}_{\mathrm{fr}}\right)$ forms of phytochrome and negatively regulates signaling (Fankhauser et al., 1999). Additionally, PKS1 is a direct phosphorylation substrate of PhyA (Fankhauser et al., 1999). There 
are four members of the PKS protein family (PKS1 to 4); both PKS2 and PKS4 have been shown to interact with PHYA (Patricia Lariguet et al., 2003; Patricia Lariguet \& Dunand, 2005; Schepens, Boccalandro, Kami, Casal, \& Fankhauser, 2008).

PKS1, PKS2, and PKS4 have all been shown to be important for the phototropic pathway; plants lacking the PKS proteins exhibit a loss-of-function in this response, while double and triple mutants exhibit a greater loss of function

phenotype (P. Lariguet et al., 2006). Studies by Demarsey and colleagues (2012) have shown that PKS4 is a direct substrate of the phot1 kinase domain. The phosphorylation of PKS4 is not a prerequisite for phototropic responsiveness, though it was shown that an increase in phosphorylated PKS4 leads to attenuation of the response (Demarsy et al., 2012). PKS1 has been shown to be a plasma membrane-associated protein in dark and light conditions and to physically interact with both phot1 and NPH3, suggesting that these three proteins might form a complex at the plasma membrane (P. Lariguet et al., 2006). Interestingly, PKS2 also plays a role in leaf flattening and positioning, which is another NPH3-dependent phot-mediated response (de Carbonnel et al., 2010).

\section{Cytosolic calcium is a possible intermediary signal within the}

\section{phototropic pathway}

In 1999 Baum and colleagues found that cytosolic calcium levels changed in response to exposure to blue light. Additionally, they demonstrated that this 
change was phot-dependent (Baum, Long, Jenkins, \& Trewavas, 1999). Using pharmacological compounds, Folta and colleagues (2003) were able to show that blocking calcium influx had an adverse response on the phot1-dependent portion of blue-light-induced hypocotyl growth inhibition. However, these pharmacological experiments did not show a change in the phototropic bending response (Folta, Lieg, Durham, \& Spalding, 2003). Yet by measuring changes in calcium fluxes, it was shown calcium influx occurs coincident with the phototropic bending response (Babourina, Godfrey, \& Voltchanskii, 2004). More recently, it has been shown that the influx of calcium is necessary for the bending response in high blue light and that phot2 is required for this response (X. Zhao et al., 2013).

Increase in cytosolic calcium can come from either the extracellular space or from intracellular compartments such as the vacuole (Sanders, Pelloux, Brownlee, \& Harper, 2002). Harada and colleagues (2003) have shown that action of both phot1 and phot2 is needed for blue light induced increase of cytosolic calcium (A. Harada, Sakai, \& Okada, 2003). However, where the new cytoplasmic calcium comes from varies depending upon the intensity of light. Under low intensity blue light where only phot1 is active, calcium enters the cell from the extracellular space by means of calcium channels, while in higher intensity light where both phot 1 and phot2 are active, there is additional influx of calcium from internal stores (Akiko Harada \& Shimazaki, 2007).

As briefly mentioned before and will be discussed in more detail in the following section, the plant hormone auxin in critical for plant development (Y. Zhao, 
2010). It has been shown that upon blue light irradiation, a lateral redistribution of auxin occurs, leading to the bending response (Esmon et al., 2006). One of the auxin transporters involved in this redistribution is PIN1, which is regulated by the protein kinase PINOID (Blakeslee, Bandyopadhyay, Peer, Makam, \& Murphy, 2004; Friml et al., 2004). Benjamins and colleagues (2003) found that PINOID can physically interact with two proteins involved in calcium binding: $\mathrm{TCH} 3$, a calmodulin-related protein, and AtPBP1 (Arabidopsis PID-BINDING PROTEIN1), a calcium binding protein. This finding lends support to a hypothesis that calcium plays a role in the phototropic response (Benjamins, 2003). Previous work had already identified the crosstalk between auxin and calcium signaling. In maize coleoptiles, upon unilateral blue light stimulation, a differential gradient of calcium is established across the coleoptile, similar to what has been observed for auxin (Felle, 1988; Gehring, Williams, Cody, \& Parish, 1990). More recently, Zhao and colleagues (2013) have shown that abolishing polar auxin transport adversely affects the bending response in the phototropic pathway and that the amount of IAA and cytosolic calcium are correlated, further supporting the finding that crosstalk between auxin and calcium signaling occurs during phototropism (X. Zhao et al., 2013). An interaction between PKS1 and Calmodulin4 (CAM4) has been suggested by Zhao and colleagues (2013) as a possible link between auxin, calcium, and phot signaling.

\section{ENHANCED BENDING1 (EHB1), and ADP-RIBOSYLATION FACTOR GTPase-ACTIVATING PROTEIN (ADG12) are NPH3-}




\section{interacting proteins shown to be involved in the phototropic}

\section{response}

EHB1 was identified as an NPH3-interacting protein via a yeast three-hybrid assay and confirmed by co-immunoprecipitation (Knauer, Dummer, Landgraf, \& Forreiter, 2011). Interestingly, ehb1 mutants show an increased phototropic and gravitropic responses. EHB1 was shown to preferentially interact with the BTB domain containing amino-terminal of NPH3 (Knauer et al., 2011). This suggests that EHB1 may compete with CUL3 for binding to the NPH3 BTB domain.

EHB1 contains an amino terminal C2/CalB binding domain, while the carboxyl terminal region shows homology to the ADP-ribosylation factor GTPaseactivating protein (ARF-GAP) family (Dümmer et al., 2016; Knauer et al., 2011; Rodriguez et al., 2014). In fact, ADP-RIBOSYLATION FACTOR GTPaseACTIVATING PROTEIN12 (ADG12), shows high sequence similarity to EHB1 and, additionally, contains a C2 domain (Dümmer et al., 2016; Knauer et al., 2011). Unlike EHB1, however, adg12 mutants exhibit a loss of function in the phototropic and gravitropic response. Like EHB1, ADG12 has been shown to physically interact with NPH3 (Dümmer et al., 2016; Michalski, Dümmer, Galland, \& Forreiter, 2017).

Since EHB1 and ADG12 both contain a C2 calcium-binding domain, it was important to determine what, if any, connection exist between these proteins and calcium in the regulation of both gravitropic and phototropic responses. Though exogenous addition of calcium affected both gravitropic and phototropic 
responses in wildtype seedlings, only the gravitropic response was impacted by addition of exogenous calcium to ehb1 and adg12 mutants (Dümmer et al., 2016; Michalski et al., 2017). This led the researchers to conclude that phototropic signaling through EHB1 and ADG12 occurs through means other than calcium (Dümmer et al., 2016; Michalski et al., 2017).

\section{A potential role for Heterotrimeric G proteins in phototropism}

Heterotrimeric proteins are found in a variety of eukaryotic organisms and consist of three proteins, Ga, G $\beta$, and Gy (Urano, Chen, Botella, \& Jones, 2013). These proteins form a complex and are active in a number of signaling pathways (Urano et al., 2013). An A. thaliana G $\beta$ (AGB1) has been shown to physically interact with the amino-terminal region of NPH3 (Kansup, Tsugama, Liu, \& Takano, 2014). Interestingly, an agb1 loss-of-function mutant shows a reduction in phototropic bending response, lending to the hypothesis that it may be involved in this response as well (Kansup et al., 2014). Further studies are however needed to connect $G$ proteins to the overall phot-dependent signaling pathway discussed here.

\section{Protein phosphatases have a variety of functions within phototropic signal transduction}

As mentioned earlier, a yet-to-be-identified type 1 protein phosphatase (PP1) appears responsible for the blue light-induced phot-dependent dephosphorylation of NPH3 (Pedmale \& Liscum, 2007). Interestingly, mutants defective in RCN1 (ROOT CURLING IN N-NAPHTHYLPHTHALAMIC ACID1), a subunit of type 2A 
protein phosphatase (PP2A), have been shown to exhibit enhanced phototropic bending as well as increased stomatal opening response (Tseng \& Briggs, 2010). RCN1 has been shown to interact with the amino-terminal portion of phot2 and dephosphorylate phot2. These results suggest that RCN1 action increases the inactive (phosphorylated) phot2 pool, thereby leading to increased phot2dependent responses (Tseng \& Briggs, 2010). However, rcn1 mutations have shown no impact on phot1-mediated responses (Tseng \& Briggs, 2010).

\section{Auxin Transport}

As previously mentioned, the plant hormone auxin (indole acidic acid) is critical in plant development and is involved in many different growth responses. One of these responses involves tropic stimulation. Under natural conditions auxin generated at the tip of the hypocotyl/coleoptile is transported by several different proteins in a polar manner; however, upon tropic stimulation, in addition to polar auxin transport, auxin is laterally transported across the hypocotyl/coleoptile, which has been described in the Chlodony Went Theory (Chlodony and Went, 1937; Ha, Jun, \& Fletcher, 2010; McSteen, 2010; Peer, Blakeslee, Yang, \& Murphy, 2011).

As the $\mathrm{pH}$ inside the cell is less acidic, as opposed to the extracellular space, auxin can be found either protonated (IAAH) or deprotonated (IAA-) depending on the location. Transport of auxin into cells can occur by naturally diffusing across the membrane as well as with the assistance of the auxin influx carriers AUXIN RESISTANT/LIKE AUXIN RESISTANT (AUX/LAX) proteins (M. J. Bennett et al., 1996; Zazímalová, Murphy, Yang, Hoyerová, \& Hosek, 2010). 
However, auxin needs to be actively transported out of the cell, and this is done with the aid of auxin efflux transporters, the PIN-FORMED (PIN) protein family and ABCB (ATP-binding cassette superfamily of transporters, B type/MDR/phosphoglycoprotein (PGP)) (Zazímalová et al., 2010).

\section{Auxin-Transport into the Cell}

As previously mentioned, due to the $\mathrm{pH}$ difference between the cytosol and the extracellular space, the protonated version (IAAH) of auxin can easily diffuse across the plasma membrane. However, due to the charge of the deprotonated version (IAA-) of auxin, transport is facilitated by use of $\mathrm{H}+$ symporters. These symporters were identified as auxin influx carriers by mutant screens and pharmacological studies (M. J. Bennett et al., 1996; Parry et al., 2001). While an agravitropic phenotype has been repeatedly seen in mutants of these genes (M. J. Bennett et al., 1996), no change in the phototropic phenotype has been identified. However, Stone and colleagues (2008) demonstrated a role for AUX1 in phototropism that is NPH4/ARF7-dependent. This aphototropic phenotype is not seen in seedlings with normal auxin responsiveness, but in a nph4/arf7 background IAAH diffusion across the membrane is not sufficient influx of auxin within the cell (Stone et al., 2008).

\section{Auxin efflux is facilitated in concert through proteins in two different gene families}

Auxin efflux is facilitated by members from the ABCB (ATP-binding cassette superfamily of transporters, B type/MDR/phosphoglycoprotein (PGP)) 
(Zazímalová et al., 2010) family, ABCB 1, 4 and 19 to be more specific. Despite the fact that auxin transport happens in a polar fashion (Friml, 2003), these proteins have not been not show to be polarly localized (Cho, Lee, \& Cho, 2007; Lewis, Miller, Splitt, Wu, \& Spalding, 2007). Only mutants deficient in ABCB19 exhibit a phototropic phenotype that differs from wild-type seedling, and, interestingly, the phenotype displayed is a gain-of-function response (Nagashima et al., 2008; Noh, Bandyopadhyay, Peer, Spalding, \& Murphy, 2003). The findings by Christie and colleagues (2011) further link ABCB19 to the phototropic pathway in a study that found upon blue light-stimulation, ABCB19 is a target of phot 1 kinase activity and that this phosphorylation inhibits its auxin efflux activity (John M. Christie et al., 2011). Additionally, in etiolated seedlings it has been shown that ABCB19 inhibits PIN1 cycling, thus keeping PIN1 polarly localized (Titapiwatanakun et al., 2009).

The second family of proteins involved in auxin efflux is the PIN-FORMED (PIN) gene family, named due to the pin-shaped inflorescent phenotype observed in mutant screens (Okada, 1991). The PIN family contains 8 members, consisting of two classes of proteins, the long PINS (PIN1-4 and PIN7) and the short PINS (PIN5-6 and PIN 8). The short PINS have been shown to localize to the ER membrane, whereas the long PINS have been shown to mediate polar auxin efflux in various cell types (T. Bennett, 2015). Research has shown that PIN1 is primarily polar-localized within the cell as is critical for auxin efflux in shoots, while PIN2 shows similar cellular localization patterns but has been shown to function primarily within roots (Grunewald \& Friml, 2010; E. Liscum et al., 2014). 
The PINS have been shown to function in many plant growth and development responses like gravitropism and organogenesis, as well as phototropism (T. Bennett, 2015). In roots Wan and colleagues (2012) have shown PIN2 relocalization in a blue light and phot1-depdendent manner (Y. Wan et al., 2012). Additionally, it has been shown that not only do pin3 mutants show a moderate loss-of-function phototropic phenotype, but they show lateral relocalization in response to blue light (Ding et al., 2011; Friml, Wiśniewska, Benková, Mendgen, \& Palme, 2002).

\section{Regulation of PIN proteins involves intracellular cycling, phosphorylation and members from the AGC protein kinase family}

Localization and function of the PIN proteins has been shown to involve protein recycling and phosphorylation by two proteins in the AGCV111 protein kinase family. D6 PROTEIN KINASE (D6PK), a member of the AGC 1 protein kinase family, has been identified to be polarly localized and necessary for polar auxin transport through a mutant screen of seedlings lacking the D6PK protein (Zourelidou et al., 2009). Furthermore, Zourelidou and colleagues identified that PIN is a substrate of D6PK kinase activity, resulting in increased PIN activity at the plasma membrane. More recently D6PK has been implicated in phototropic signal transduction as seedlings lacking this protein exhibit a loss-of-function bending response (Willige et al., 2013). 
A second kinase involved in regulating PIN function has been shown to be involved in polarity cycling of PIN protein between the apical and basal cell locations named PINOID (PID) (Friml et al., 2004). Two serine/threonine protein kinase, WAG1 and WAG2, have been found to be similar to PID (Santner \& Watson, 2006). PID has been shown to phosphorylate PIN proteins, signaling for their polarity switch within the cell (Huang et al., 2010). Interestingly, Ding and colleagues (2011) were able to show that in seedlings lacking PID/WAG1/WAG2, PIN3 localization was unchanged regardless of blue light stimulation. Furthermore, these seedlings also exhibited a lack of phototropic response, implicating the involvement of these protein kinases along with PIN proteins in the lateral redistribution of auxin leading to the bending response (Ding et al., 2011).

In addition to cycling between the apical and basal locations within the cell, PIN proteins have also been shown to be recycled by the action of GNOM, which is an ARF GEF (ADP-RIBOSYLATION FACTOR GUANINE-NUCLEOTIDE EXCHANGE FACTOR), recycling PINs from the plasma membrane through vesicle trafficking (Niko Geldner et al., 2003). Seedlings with a partial loss-offunction allele: gnom $^{R 5}$ (N. Geldner, 2003) display a defect in their phototropic bending response, THUS linking GNOM recycling of PIN proteins to phototropic signal transduction leading to a change in auxin distribution (Ding et al., 2011).

\section{Perception of Auxin}

Sections below written by Kyle Willenburg 
As described above, phot1 initiates a set of molecular signaling events that lead to the formation of an auxin gradient within the hypocotyl, which is prerequisite to phototropism. In the following sections we will detail the mechanisms by which the auxin perception and response occur.

\section{SCF $^{\mathrm{TIR} 1 / \mathrm{AFB}}$ Functions as an Auxin Perception Complex}

Auxin perception is necessary to transduce the blue-light dependent auxin gradient into differential growth—phototropic growth. Several systems of auxin perception have been discovered to date, SCF ${ }^{\text {TIR1/AFB }}$ complex, S-phase-kinaseassociated protein 2 (SKP2), and the now more controversial Auxin Binding Protein 1 (Dezfulian et al., 2016; Grones \& Friml, 2015; R. M. Harper et al., 2000; Strader \& Zhao, 2016). Due to its specificity the SCF TIRT1/AFB complex, functions as a sensitive receptor of auxin concentration that ultimately leads to signal transduction through activation of specific tropic responsive genes. The $\mathrm{SCF}^{\mathrm{TIR} 1 / \mathrm{AFB}}$ complex is comprised of three core component proteins: Skp1/ASK1, Cullin1, and an F-box (TIR1 or an AFP) protein, as well as the RBX1 and an E2 ligase proteins necessary for enzymatic activity of the complex (Grones \& Friml, 2015). Together, the proteins of the SCF complex function as a nuclear localized E3 ubiquitin ligase to ubiquitinate repressor proteins (Aux/IAAs), marking them for degradation and ultimately leading to transcriptional de-repression of auxin responsive transcriptional factors (ARFs) (Grones \& Friml, 2015). 


\section{SCF Complex Proteins: Who they are and what they do}

Arabidopsis SKP1-Like (SKP1/ASK1) functions to connect the SCF complex to the F-box protein. CULLIN1 (Cul1) provides a scaffold for the ubiquitin ligase to associate with, connecting ASK1 and RBX1 proteins together. RING Like BOX PROTEIN 1 (RBX1) connects Cul1 to the E2 ubiquitin ligase. TRANSPORT INHIBITOR RESPONSE 1 (TIR1) and AUXIN SIGNALING F-BOX PROTEINS (AFBs) are F-box proteins that function as coreceptors of auxin along with Aux/IAAs.

\section{Auxin Signaling: Linking Auxin Perception to Activation}

The first component of the auxin responsive signaling response system is the TIR1/AFB auxin co-receptor. The second component is the Auxin/INDOLE-3ACETIC ACID (Aux/IAA) protein auxin co-receptors (Grones \& Friml, 2015), and the third the AUXIN RESPONSE FACTOR (ARF) transcription factors that bind to specific DNA sequences, dubbed auxin responsive elements (AuxREs) in the promoters of auxin responsive genes (Ulmasov, Hagen, \& Guilfoyle, 2017). There are 29 AUX/IAAs (Guilfoyle, 2015; J Kim, Harter, \& Theologis, 1997; E. Liscum \& Reed, 2002), four TIR1/AFBs, and 23 ARFs in Arabidopsis (Okushima et al., 2005). Both ARFs and Aux/IAA transcription factors have been shown to hetero-homodimerize, forming ARF-ARF, ARF-Aux/IAA, or Aux/IAA-Aux/IAA associations by means of electrostatic interactions in their Carboxyl-terminal located PB1 domain, historically referred to as domain III/IV (Guilfoyle, 2015; M. Han et al., 2014; Korasick et al., 2014). ARFs additionally contain an N-terminal 
DNA binding Domain (DBD) that recognizes auxin responsive gene promoter regions (Boer et al., 2014; Guilfoyle, 2015).

\section{The Mechanisms by which the SCF ${ }^{\text {TIR1/AFB }}$ Complex Senses}

\section{Auxin and Activates Gene Expression}

The SCF ${ }^{\mathrm{TIR} 1} / \mathrm{AFB}$ complex functions to ubiquitinate Aux/IAA proteins, targeting them for $26 \mathrm{~S}$ proteasomal degradation. When auxin levels are low, Aux/IAA repressor proteins are bound to ARF transcriptional activators that are associated with (AuxREs), inhibiting transcription of auxin responsive genes (Lavy \& Estelle, 2016). However, when auxin concentrations are relatively high, Aux/IAAs bind to auxin in conjunction with the F-box coreceptor TIR1, which in

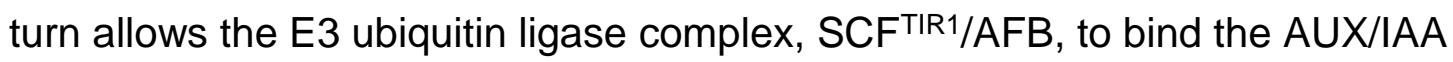
proteins, polyubiquitinating and marking the Aux/IAA proteins for degradation. Once the Aux/IAA proteins are degraded and their ARF binding repression eliminated, ARFs are free to dimerize and induce the expression of the auxin responsive genes they are associated with. The regulatory method of repression/de-repression described above provides a sensitive and elegant mechanism for regulating differential growth.

\section{Transcriptional Modification Induced by the Polar Auxin}

\section{Gradient Leads to Phototropism}

Auxin-regulated gene expression specific to phototropism requires ARF7 for phototropic signaling (Stowe-Evans, Harper, Motchoulski, \& Liscum, 1998)(E. Liscum, 1995)(R. M. Harper et al., 2000)(E Liscum \& Briggs, 1996)(E. Liscum, 
1995). ARF7 was initially identified as NON-PHOTOTROPIC HYPOCOTYL 4 (NPH4) in a fast-neutron mutant screen (E. Liscum, 1995; E Liscum \& Briggs, 1996). At the time NPH4 was not yet characterized as an auxin responsive factor, but phototropic and gravitropic assays soon demonstrated a likely role in modulating the differential growth responses associated with tropic curvature (E Liscum \& Briggs, 1996). Phenotypic studies of $n p h 4$ mutants suggested that $\mathrm{NPH} 4$ functions in the differential growth of tropic curvature and further suggested that NPH4 is a modulator of auxin-dependent differential growth (Stowe-Evans et al., 1998). Map-based cloning of NNPH4 revealed that it encodes ARF7 and produced the first genetic links between ARF biochemistry and physiology (R. M. Harper et al., 2000).

In a screen that identified mutants with defects in auxin induced curvature, IAA19/MSG2 was identified (Tatematsu, 2004). In the same study it was shown that NPH4/ARF7 and IAA19 interact with each other and that in the nph4-1 mutant, IAA19 showed a drastic decrease in transcript level, although it normally increases rapidly in response to auxin (Tatematsu, 2004). NPH4 and IAA19 likely function in a negative feedback loop where auxin causes NPH4 to upregulate its own repressor. It is also worth noting that in plants carrying a dominant mutation in IAA19 that stabilizes the AUX/IAA19 protein, a reduction in auxin induced gene expression is observed (Tatematsu, 2004).

\section{Auxin Triggered Tropic Genes}

While research has focused on and generally been effective at elucidating the mechanisms of phototropic light perception and some understanding of the 
formation of the auxin gradient that precedes and induces the phototropic genes, the specific genes causative for the downstream mediation of the phototropic response have not been fully identified, nor their roles well characterized. The auxin gradient that leads to differential expression of auxin-responsive genes in regions of the hypocotyl necessitates comparing transcripts between opposing flanks of phototropically stimulated hypocotyls to determine which genes are altered by phototropic signaling. This impediment alone would not be a true roadblock if much of the current phototropic research was not conducted in the model plant organisms Arabidopsis thaliana (Esmon et al., 2006). Esmon and colleagues (Esmon et al., 2006) circumvented some of the difficulties associated Arabidopsis thaliana by using Brassica oleracea as a test organism. By collecting mRNA from opposing flanks of light-stimulated $B$. oleracea seedlings and probing an $A$. thaliana microarray, eight genes were identified that had AuxREs and increased transcript levels where increase in auxin levels was observed (Esmon et al., 2006). What is more, none of the eight genes, termed tropic stimulus induced (TSI) genes, displayed differential accumulation in a nph4-1 null background (Esmon et al., 2006). It is worth discussing each of the genes or gene families in some detail below.

\section{Expansins}

Of the TSI genes identified two genes, EXPANSIN A1 (EXPA1) and EXPANSIN A8 (EXPA8) can be most easily fit into an elegant model for phototropism (Esmon et al., 2006). Expansins promote rapid, within seconds, cell wall creep that allows for cell wall elongation (A. C. Li \& Cosgrove, 2001; Y. B. Park \& 
Cosgrove, 2012; Sheng Yuan, Yajun Wu, 2001). The expansin superfamily contains four families, 26 alpha expansins (A), 6 beta (B) expansins, 3 expansinlike A, and 1 expansin-like B (Y. Li et al., 2002; Sampedro \& Cosgrove, 2005). The proteins themselves are usually $250-275$ amino acids long with two domains and a signal peptide to permit its secretion to the apoplast (Yennawar, $\mathrm{Li}$, Dudzinski, Tabuchi, \& Cosgrove, 2006). Expansins are thought to mediate cell growth by functioning to weaken noncovalent bonds that exist between cellulose and hemicellulose, thereby permitting polymers to move as turgor pressure puts tension on the cell wall (D. J. Cosgrove, 1997; Daniel J. Cosgrove, 2000). Expansins are an important piece of the acid-growth hypothesis, which states that auxin induces a decrease in $\mathrm{pH}$, leading to subsequent cell-wall loosening (Rayle \& Cleland, 1992). Cell wall loosening is highly dependent on the $\mathrm{pH}$ of the cell wall with maximal cell wall loosening occurring between $\mathrm{pH} 3.5$ and $\mathrm{pH} 4.5$ (Mcqueen-mason, Durachko, \& Cosgrove, 1992; Rayle \& Cleland, 1992). It has long been accepted that auxin and cell wall acidity are interconnected (Rayle \& Cleland, 1970). More recently, the $\mathrm{H}^{+}$-ATPases (AHAs) have been identified in the process of cell wall acidification (Baxter, 2003; Haruta et al., 2010) and the link between auxin and wall acidification more clearly established by the demonstration that auxin stimulates phosphorylation of AHAs (Takahashi, Hayashi, \& Kinoshita, 2012). In this context it is important to note that expansin proteins are activated by acidic conditions (Mcqueen-mason et al., 1992). The finding that two expansins (EXPA1 and EXPA8) exhibit increased transcript accumulation in response to tropic stimulation in the elongating hypocotyl flank 
prior to absorbed curvature early on (Esmon et al., 2006) is certainly consistent with these molecules directly regulating the differential growth response.

\section{Auxin Conjugating Enzymes GH3.5/WES1 and GH3.6/DFL1 May Attenuate Auxin Induced Transcription During Tropism}

Signal attenuation is critical for most signaling events, and this is certainly true of phototropism. Because auxin acts to initiate the transcription of genes, either removing auxin or preventing its signaling influences could serve as rapid mechanism to halt such transcription. In plants the $\mathrm{GH} 3$ family of proteins have been shown to mediate conjugation of Ala, Asp, Phe, and Trp to auxin as a method of regulating auxin activity (P. E. Staswick, 2005; Westfall, Herrmann, Chen, Wang, \& Jez, 2010). Transcripts for two GH3 proteins, GH3.5/WES1 and GH3.5/DFL1, have been shown to accumulate in response to tropic stimulation (Esmon et al., 2006). Interestingly, these GH3 transcripts exhibit maximal accumulation coincident with the peak tropic response, suggesting that they may indeed down-regulate the response (Esmon et al., 2006). These two proteins have been shown in Arabidopsis to conjugate amino acids to IAA (J. E. Park et al., 2007; P. E. Staswick, 2005; Paul E Staswick et al., 2002). A dominant overexpressing mutant of the GH3.6/DFL1 gene (dfl1-D) was shown to have reduced shoot elongation in blue, red, and far-red light, as well as root phenotypes (Nakazawa et al., 2001). Additionally, GH3.5/WES1 knockout and dominant overexpressing lines manifested interesting phenotypes: the wes 1 knockout showed increased hypocotyl length, while wes1-d dominant- 
overexpressing line showed decreased hypocotyl elongation compared to wildtype plants (J. E. Park et al., 2007). Taken together, current evidence supports a hypothesis for a role of $\mathrm{GH} 3$ proteins in the regulation of hypocotyl elongation, including the differential elongation response observed in tropisms by regulating the auxin concentration fluctuations.

\section{SMALL AUXIN UP RNAs (SAURs) and Tropisms}

Among the classes of early auxin responsive genes, SAURs are the largest family (Ren \& Gray, 2015). SAURs were originally identified while screening for genes rapidly induced by auxin in soybean hypocotyls (McClure \& Guilfoyle, 1987), and present bioinformatic analyses have catalogued 81 SAURs in Arabidopsis (Hagen \& Guilfoyle, 2002). At least one SAUR to date (SAUR50) has been shown to be tropically induced in the elongating flanks of growing hypocotyls (Esmon et al., 2006). The SAUR genes usually lack introns (Wu et al., 2012) and have two or more AuxREs (Jain, Tyagi, \& Khurana, 2006). SAUR genes encode small proteins with a predicted range in molecular masses from 9.3 to $21.4 \mathrm{kDa}$ that do not contain any characterized motifs with known biochemical function (Hagen \& Guilfoyle, 2002). SAUR proteins are predicted to localize to the nucleus, cytosol, mitochondria, chloroplasts, and on the plasma membrane (Wu et al., 2012). Multiple sequence alignments have revealed that SAUR proteins have a highly conserved central domain (CDD) specific to SAURs that is essential to their function and highly conserved (Marchler-Bauer et al., 2013; Ren \& Gray, 2015). 
Several SAURs have been implicated in hypocotyl elongation in a number of recent studies (Chae et al., 2012; Qiu et al., 2013; Angela K. Spartz et al., 2017; Angela K Spartz et al., 2012; Wu et al., 2012). The method by which SAURs mediate an elongation response is not yet well characterized, but it has been recently suggested that SAURs may be involved in acid growth. For example, SAUR19 appears to upregulate plasma membrane $\mathrm{H}^{+}$ATPases through a double negative regulation where it inhibits protein phosphatases that are responsible for repression of the $\mathrm{H}^{+}$ATPases (A. K. Spartz et al., 2014). This finding is particularly exciting knowing that expansins are activated when cell wall $\mathrm{pH}$ is decreased as discussed above. This information together with the finding that SAUR50 is transcriptionally upregulated upon tropic stimulation is indeed intriguing (Esmon et al., 2006).

\section{HATs and Auxin-Induced Hypocotyl Elongation}

HAT2 was identified as a tropic stimulus induced gene (Esmon et al., 2006) and has roles that fit into the model of tropic growth so far discussed. HAT2 contains a homeodomain and a leucine zipper domain and is classified as a member of the HD-Zip II subfamily which contains 10 members (Ciarbelli et al., 2008; Sawa et al., 2002). HAT2 was shown to be rapidly induced by auxin (within 10 minutes) (Sawa et al., 2002). Interestingly, 35S::HAT2 overexpression lines displayed longer than normal hypocotyls because of increased cell elongation but not cell proliferation (Sawa et al., 2002). Certainly this phenotype is consistent with differential up-regulation of HAT2 in the elongating flank of a tropically-stimulated seedling being positively correlated with increased elongation in that flank 


\section{SKS1 is a Glycosylphosphatidylinositol-Anchored (GPI-}

\section{Anchored) Protein}

Though identified as a TSI gene, expression of SKS1 was not examined in detail by (Esmon et al., 2006). SKS1 belongs to a 19 member gene family SKS (for SKU5 Similar) which encode structurally related proteins with homology to the multiple-copper oxidases ascorbate oxidase and laccases though they lack copper binding motifs and are thus predicted to confer no catalytic activity (Sedbrook, Carroll, Hung, Masson, \& Somerville, 2002). SKU5, the founding member of the family is a GPI anchored protein (Georg H.H. Borner, Kathryn S. Lilley, Timothy J. Stevens \& Borner, 2003) involved in anisotropic growth, potentially through regulation of cell expansion (Georg H.H. Borner, Kathryn S. Lilley, Timothy J. Stevens \& Borner, 2003; Sedbrook et al., 2002). Though much more research is required into the mechanisms by which SKS proteins function, what little is known implicates SKS1 as a potential player in tropic growth responses.

\section{A bHLH May Serve as an Additional Regulator of Tropic Induced}

\section{Hypocotyl Elongation}

Previous work has indicated that the expression of a basic helix-loop-helix protein $(\mathrm{bHLH})$ has expression that is induced by both photo and gravitropism (Esmon et al., 2006). bHLH proteins were initially discovered as DNA binding proteins in animals (Murre, McCaw, \& Baltimore, 1989), then in maize (Ludwig, Habera, Dellaporta, \& Wessler, 1989) and yeast (Berben, Legrain, Gilliquet, \& 
Hilger, 1990). Within plants bHLH proteins have been shown to be the second largest family of transcription factors (Mao, Dong, Li, Liu, \& Ma, 2017). Most of the recent research conducted on this family has been conducted at the whole genome level (Carretero-Paulet et al., 2010; X. Li et al., 2006; Mao et al., 2017; Pires \& Dolan, 2010) yet few studies have begun to examine the responses these transcription factors regulate. One study showed that PIF3, a bHLH that interacts with the red light photoreceptors phyA and phyB, was involved in regulating phyA and phyb signaling (Ni, Tepperman, \& Quail, 1998). More recently it was shown that overexpression of PACLOBUTRAZOL RESISTANCE1 (PRE1)/bHLH134 promotes elongated hypocotyls and early flowering (S. S. Lee et al., 2006). Overexpression of the four most closely related bHLHs to PRE1 (PRE2-5) results in similar phenotypes (S. S. Lee et al., 2006). PRE2 is bHLH134, the gene shown to be tropic stimulation induced (Esmon et al., 2006). Further, bHLH134 (called BNQ2 in this study) was shown to heterodimerize with and negatively regulate other bHLH transcription factors, specifically binding to LONG HYPOCOTYL IN FAR-RED LIGHT1 (HFR1) (Mara, Huang, \& Irish, 2010). All this evidence taken together points towards bHLHs involvement in photomorphogenesis and likely in the case of bHLH134, the differential cellular elongation of tropism. 


\section{Other Modifiers of Phototropism}

\section{Phytochrome A: Phytochromes are Red/Far-Red Reversible}

\section{Light Receptors}

phototropin, as discussed so far, is the photoreceptor responsible for perception of blue light and initiates signaling events that progress to the development of a bending response. However, phototropins, though the primary receptor responsible for phototropic light perception, rely on phytochromes, the red/far-red light-sensing family of light receptors to modulate the bending response. In plants phytochromes are dimerized large, $124 \mathrm{kDa}$, proteins with, broadly speaking, two modules: an $\mathrm{N}$-terminal photosensory module that contains a linear light-absorbing tetrapyrrole and a C-terminal regulatory module important for dimerization and signal output (Franklin \& Quail, 2010; Møller, Ingles, \& Whitelam, 2002; Rockwell, 2006).

Phytochromes are grouped together in a small gene family containing five members PHYA-E (Clack, Mathews, \& Sharrock, 1994). The five phytochromes (A-E) are divided into two groups: phyA is the sole member of the group I being quickly turned over in high light and serving a primary function in very low fluence responses, group II phys, phyB-C with phyB function as the dominant receptor are light stable and mediate responses such as shade avoidance and flowering time (Fankhauser, 2001). As a group, phytochromes mediate many important responses: germination, de-etiolation, gravitropic orientation, shade avoidance, stomatal development, circadian clock entrainment, flowering, and photoperiodic 
perception (Fankhauser, 2001; Franklin \& Quail, 2010; Chitose Kami, Lorrain, Hornitschek, \& Fankhauser, 2010). In addition to these commonly described phytochrome-mediated responses it is well established that phototropic modulation by phytochromes occurs (Emily L. Stowe-Evans, Darron R. Luesse, Stowe-Evans, Luesse, \& Liscum, 2001; Hughes, 2013; Patricia Lariguet \& Fankhauser, 2004; Parks, Quail, \& Hangarter, 1996; Rosler, Klein, \& Zeidler, 2007). In fact, a number of phytochrome dependent modulations of phototropin signaling have been observed: modulation of chloroplast movement (DeBlasio, Mullen, Luesse, \& Hangarter, 2003), far-red-light hypocotyl growth inhibition (Rosler et al., 2007), pulses of red light enhance phototropism phyA dependently (Emily L. Stowe-Evans, Darron R. Luesse et al., 2001; Parks et al., 1996), phyA is necessary for gravitropic abrogation in low fluences of blue light, phyA is necessary for blue light-induced hypocotyl growth inhibition in low to moderate light (Rosler et al., 2007), that both phyA and phyB modulate phototropism without red-light pretreatment, and that phyA functions in the attenuation of phototropism under very high fluence rate phototropism (C. Kami et al., 2012; Tsuchida-Mayama et al., 2010; C. W. Whippo \& Hangarter, 2004). As noted above, phototropin signaling is modulated by the phytochromes in many physiological responses and that phyA seems to serve a primary function in many of the phototropic modulations. Genetic and physiological screening has generally been effective at identifying the modulatory influences of phytochromes on phototropin signaling, but mechanistic evaluation has proven more difficult to elucidate. The localization of phyA and the development of genetic lines that 
influence phyA localization have proven valuable in describing, not the mechanism of action, at least the cellular location. phyA exists as at highly abundant levels in the cytosol in darkness, and upon light perception is quickly shuttled into the nucleus by two proteins FR-elongated hypocotyl 1 (FHY1) and FHY1-like (FHL). In a study by Rosler and colleagues (2007), in a null fhy $1 /$ fhl background where phyA cannot be localized to the nucleus, multiple phy $A$ dependent responses were still observed while several were impaired, which implies that phyA in the cytosol and phyA that is localized to the nucleus both mediate different sets of responses and supports a hypothesis where phytochrome has a cytosolic and nuclear signaling route. It was shown that farred hypocotyl growth inhibition was impaired but not blue-light-induced hypocotyl growth inhibition, implying that phyA cannot signal far-red hypocotyl growth inhibition responses unless it is localized to the nucleus but able to modulate blue-light-induced hypocotyl growth inhibition from the cytosol (Rosler et al., 2007). Interestingly, they further showed that cytosolically localized phyA is able to enhance blue-light phototropism and that seedlings grown in directional blue light are able to abrogate the gravity vector and grow towards a blue light source (Rosler et al., 2007).

It is intriguing to note that many of the cytosolically modulated responses are blue-light phototropin mediated responses. There is evidence to support, though somewhat speculatively, that a more direct signaling route may exist between phot 1 and the phyA. As noted already, phot 1 is a plasma membrane associated protein and phyA is cytosolically localized, putting both proteins at least in the 
same space, making possible some level of interaction, possibly via an intermediate molecule. Red-light pulses prior to low fluence rate blue-light treatments has been shown to prevent the blue-light-induced loss of phot 1 from the membrane, a movement with a great deal of research devoted to investigating (Emmanuel Liscum, 2016), but shows that phyA regulates phot distribution (I.-S. Han et al., 2008). Other evidence exists that phot1 and phyA may cooperate in signaling at the membrane; both phyA and phot 1 interact with PHYTOCHROME KINASE SUBSTRATE 1 (PKS1) (Fankhauser et al., 1999; Chitose Kami et al., 2014; P. Lariguet et al., 2006). Present research has not conclusively demonstrated any more direct interaction between phyA and phot1, but the current evidence is tantalizing nevertheless. When looking in other plant lineages, phots and phys have been clearly shown to interact, so it would be unsurprising to find that a similar relationship exists in higher plants. For instance, ferns and algae mediate phototropism by means of a neochrome photoreceptor (Hughes, 2013; Nozue et al., 1998; Suetsugu, Mittmann, Wagner, Hughes, \& Wada, 2005), which is essentially a phototropin and phytochrome chimeric protein, in the moss Physcomitrella patens $P H Y 4$ was shown to interact with Physcomitrella phototropins in yeast two-hybrid $(\mathrm{Y} 2 \mathrm{H})$ studies (Hughes, 2013). Lastly, (Jaedicke, Lichtenthäler, Meyberg, Zeidler, \& Hughes, 2012) showed that phot1 and phyA interact at the membrane in onion cells in split-YFP studies, but did not show interaction in $\mathrm{Y} 2 \mathrm{H}$ tests; as a result, the authors suggested that yeast cells may lack one or component of a larger complex, suggesting PKS1 or NPH3 as potential links between phyA and phot1. 


\section{Cryptochromes}

Cryptochromes (crys) are an additional class of blue-light absorbing flavoprotein photoreceptors belonging to a subgroup within the cry/photolyases superfamily, which are broadly distributed from bacteria to plants to humans (Chaves et al., 2011; Bobin Liu et al., 2016). The superfamily that crys belong to contains the more well-known proteins, the photolyases, which are responsible for repair of UV induced pyrimidine-pyrimidine DNA adducts by using blue light as an energy source for the repair (Chaves et al., 2011; Sancar, 2004); cryptochromes themselves have no photolyase activity but have protein sequence homology. In Arabidopsis, there are two cryptochromes (cry1 and cry2) that have been implicated in many developmental responses during the lifecycle of plants such as photoperiod induced flowering time (Ahmad et al., 1998; Chaves et al., 2011; Bobin Liu et al., 2016), but first discovered for their role in regulating hypocotyl growth inhibition in blue-light (Ahmad \& Cashmore, 1993).

Crys facilitate physiological responses to blue through transcriptional regulation of many genes (Chaves et al., 2011; Franklin, Larner, \& Whitelam, 2005; Bin Liu, Zuo, Liu, Liu, \& Lin, 2011). Crys function by perceiving blue light and transducing the signal through other unknown intermediate proteins by means of a Cterminus domain, though the domain is not highly conserved between Arabidopsis cry1 and 2 (Chaves et al., 2011). Two methods by which crys regulate transcription have been discovered so far; crys have been shown to operate by suppressing CONSTITUTIVE PHOTOMORPHOGENIC 1 (COP1)mediated proteolytic degradation of the LONG HYPOCOTYL 5 (HY5) 
transcription factor and by binding directly to basic helix-loop-helix transcription factors (Bin Liu et al., 2011; Bobin Liu et al., 2016; H. Liu, Liu, Zhao, Pepper, \& Lin, 2012).

A number of studies have demonstrated cryptochrome roles in phototropism by evaluating lines expressing single or double mutations and in hypocotyl elongation (Lascève et al., 1999; Bobin Liu et al., 2016; Nagashima et al., 2008; Ohgishi et al., 2004; Tsuchida-Mayama et al., 2010; Craig W Whippo \& Hangarter, 2003). Cry1, cry2, phyA, and phyB mutants showed defects in high light phototropism and transcriptional activation of RPT2, suggesting that crys together with phyA and phyB may be regulating phototropism by modulating RPT2 expression, which has been shown to attenuate a negative function in hypocotyl phototropism (Inada et al., 2004; Tsuchida-Mayama et al., 2010). Additionally ABCB19 has been shown to suppress phototropism and be regulated by cryptochromes and phytochromes (Nagashima et al., 2008), representing another route that cryptochromes use to regulate phototropism.

\section{Katanin and Microtubule Reorientation is Necessary for \\ Phototropism}

In many organisms, centrosomes act to organize microtubule arrays by controlling and positioning nucleation complexes, yet higher plants lack centrosomes altogether and the mechanism by which they regulate microtubule arrays is not well understood (Bartolini \& Gundersen, 2006). At the cell cortex, higher plants construct highly ordered microtubule arrays that organize cell wall 
and growth, directing morphogenesis (Baskin, 2001; Paredez, Somerville, \& Ehrhardt, 2006). The microtubules of cortical cells are dynamically responsive to signals, most interesting to this review, light (Zandomeni \& Schopfer, 1993). In elongating hypocotyls, cortical microtubule arrays are transversely organized to the axis of growth, but blue light signals them to reorient $90^{\circ}$ within minutes (Nick, Bergfeld, Schäfer, \& Schopfer, 1990). This blue light induced microtubule reorientation is phototropin dependent, mediated by a Katanin activity, and without Katanin, phototropism is severely impaired (Lindeboom et al., 2013). Do to the severity of phototropic impediment in Katanin mutants, the regulation of Katanin by phot 1 is certainly a critical step in the phototropic signaling and further research in the mechanisms of blue-light dependent microtubule arrangement will likely prove valuable to the field as a whole. 


\section{Chapter 2 DECREASE IN PHOTOTROPIC CURVATURE RESULTANT OF SITE-DIRECTED MUTAGENESIS IN PHYTOCHROME A UBIQUITINATED LYSINE RESIDUES}

\section{Abstract}

Phytochrome A (phyA) has been shown to play important roles in the modulation of phototropin 1 (phot1)-dependent phototropism, but the mechanisms have not yet been demonstrated. NON-PHOTOTROPIC HYPOCOTL3 (NPH3), which is a phot1-interacting protein essential for phototropism, has been shown to function as an E3 ubiquitin ligase substrate adapter, serving a critical role in the ubiquitination of phot1. Previous ubiquitin remnant profiling results showed that phyA is ubiquitinated in response to blue light exposure in a NPH3-dependnent fashion. In this study we used site-directed mutagenesis (SDM) to alter the two target lysine residues to arginine to prevent ubiquitination at these sites, and then analyzed the SDM lines both biochemically and physiologically. Results from this study indicate that altering the two lysine residues to arginine results in suppression of phototropism at very high fluence rates of blue light but has no impact on very low and low fluence phototropism or far-red light-dependent hypocotyl growth inhibition, which is a well know phyA-dependent response. Western blotting indicates that SDM lines show a reduction in phyA degradation in response to blue light but not red light. 


\section{Introduction}

\section{Plants use photoreceptors to sense light and modulate growth}

Plants, being sessile, must adapt to their environment to acquire and compete for resources. Light is one of the most critical environmental resources for plants; plants use light not only as their primary energy resource, but also gather from it critical information (E. Liscum et al., 2014). Plants use light sensing to determine: day length, season, and neighbor proximity (Pudasaini \& Zoltowski, 2013; Serrano-Bueno, Romero-Campero, Lucas-Reina, Romero, \& Valverde, 2017; SMITH \& WHITELAM, 1997). The environmental light cues plants perceive facilitate directed development of nearly all aspects of plant growth including: germination, cotyledon opening, control of hypocotyl elongation, greening, petiole angle, leaf expansion, shade avoidance, and flowering, to name a few (W. R. Briggs, 2001; Fiorucci \& Fankhauser, 2017; Nemhauser \& Chory, 2002; Whitelam, Patel, \& Devlin, 1998). Light sensing is facilitated via multiple families of photoreceptors: the red/far-red sensing phytochromes (Franklin \& Quail, 2010), blue sensing phototropins (E. Liscum et al., 2014), blue sensing cryptochromes (Chaves et al., 2011), ultraviolet B sensing UVR8 (Jenkins, 2014), and blue light sensing Zeitlupe family members (Pudasaini \& Zoltowski, 2013). Notably, there is extensive crosstalk and overlap in the signaling downstream of plant photoreceptors; thereby the responses of one photoreceptor are influenced by other photoreceptors signaling (Casal, 2000, 2013; Chitose Kami et al., 2010; C. W. Whippo \& Hangarter, 2004). 
There is a steadily increasing body of data revealing that phyA and phot1 cooperatively regulate a number of responses and may even function as part of a complex (Jaedicke et al., 2012; C. W. Whippo \& Hangarter, 2004).

\section{Phytochrome A and phototropin}

Phytochromes (phys) are encoded by a small gene family; in Arabidopsis thaliana there are five phys, phyA-E, which are divided into two types. (Clack et al., 1994). Type I phytochromes, represented by phyA in $A$. thaliana, are rapidly degraded in high light and serve a primary function in very low fluence responses. Type II phys, phyB-E in A. thalina, mediate low to moderate light responses, such as shade avoidance and flowering time (Fankhauser, 2001). In addition to the many responses described above that phytochromes mediate, it is well established that phys modulate phot-dependent phototropism (Emily L. Stowe-Evans, Darron R. Luesse et al., 2001; Hughes, 2013; Patricia Lariguet \& Fankhauser, 2004; Parks et al., 1996; Rosler et al., 2007). There are other examples of blue light responses undergoing modulation by phys. For example, phyA is necessary for gravitropic abrogation in low fluences of blue light and necessary for blue light-induced hypocotyl growth inhibition in low to moderate light (Rosler et al., 2007).

How might phyA be modulating phot-dependent responses? There is evidence for a rather close mode of signaling between phot1 and phyA (R. M. R. M. Harper et al., 2001; Jaedicke et al., 2012). phot1 is a plasma membraneassociated protein, and phyA is cytosolically localized, providing for the possibility of interaction at some level, possibly via an intermediate molecule ( $R$. 
M. R. M. Harper et al., 2001; Jaedicke et al., 2012; Rsler, Jaedicke, \& Zeidler, 2010). Both phyA and phot1 interact with PHYTOCHROME KINASE SUBSTRATE 1 (PKS1) (Fankhauser et al., 1999; Chitose Kami et al., 2014; P. Lariguet et al., 2006). Evidence in higher plants does not support a direct interaction between phyA and phot1, but studies in other plant lineages show that phots and phys interact. For instance, ferns and algae mediate phototropism by a neochrome photoreceptor, which is an evolutionary chimera of phototropin and phytochrome (Hughes, 2013; Nozue et al., 1998; Suetsugu et al., 2005). In the moss Physcomitrella patens, PHY4 (the moss paralog of phyA) was shown to interact with all four Physcomitrella phots (Hughes, 2013). Lastly, A. thaliana phot1 and phyA interact at the membrane in onion cells in split-YFP studies, but have been shown to interact as native proteins in A. thaliana (Jaedicke et al., 2012). Current evidence suggests that PKS1 and/or NPH3 may serve as potential links between phyA and phot1 in planta (Jaedicke et al., 2012). phot1 is a blue light photoreceptor that requires the E3 ubiquitin ligase substrate adapter NPH3, an NRL family member, for

\section{phototropic signaling}

phot1 is a blue light photoreceptor that mediates phototropism and responses that optimize photosynthetic efficiency (E. Liscum et al., 2014). During phototropic signaling phot1 is ubiquitinated by a CULLIN3-based E3 ubiquitin (ub) ligase, $\mathrm{CRL} 3^{\mathrm{NPH} 3}$, in which NPH3 functions as a substrate adapter (Roberts et al., 2011). In the absence of NPH3 phot1 is not ubiquitinated and phototropism 
does not occur (Roberts et al., 2011). NPH3 is a founding member of the NRL (NPH3/RPT2- like) family, which has 33 members characterized by a central NPH3 domain (Inada et al., 2004; Motchoulski \& Liscum, 1999; Pedmale \& Liscum, 2007).

\section{Type 1 phytochromes are ubiquitinated and rapidly degraded}

Polyubiquitinated proteins are typically recruited to the $26 \mathrm{~S}$ proteasome, an ATPdependent protease complex that rapidly degrades proteins and is generally considered to have an important role in cell signaling (Zientara-Rytter \& Sirko, 2016). Previous studies have demonstrated that type 1 phys (e.g. phyA) is polyubiquitinated and subsequently degraded upon photoconversion to the farred light-absorbing form by treatments with red light (Jabben, Shanklin, \& Vierstras, 1989; J Shanklin, Jabben, \& Vierstra, 1987; John Shanklin, Jabben, \& Vierstra, 1989). Additional studies show that light-induced degradation of phyA involves COP1, an E3 ubiquitin ligase (Seo, Watanabe, Tokutomi, Nagatani, \& Chua, 2004). However, levels of phyA are also elevated in light grown seedlings lacking CULLIN1 (CUL1) (Quint, Ito, Zhang, \& Gray, 2005). Because COP1 acts in a CUL4 and not a CUL1 complex, these results suggest that multiple E3 ligases may be responsible for regulating phyA degradation $(\mathrm{H}$. Chen et al., 2010; Lau \& Deng, 2012). 


\section{Ubiquitin remnant profiling screen revealed phyA exhibits a}

\section{$\mathrm{CRL}^{\mathrm{NPH}}{ }^{\mathrm{N}}$-dependent ubiquitination in blue light}

A former student in the lab (Dr. Diana Roberts) performed ubiquitin remnant profiling to identify $C R L^{N P H 3}$ dependent ubiquitination events (Xu \& Jaffrey, 2011, 2013; Xu, Paige, \& Jaffrey, 2010). In brief, ubiquitin remnant profiling uses a ubiquitin branch motif antibody (K-ع-GG) to immunoprecipitate ubiquitinated peptides from trypsin digested protein lysates. Trypsin cuts at lysine or arginine residues, but when proteins are ubiquitinated, trypsin cleaves after an arginine in ubiquitin, resulting in a di-glycine motif which can be exploited to enrich for ubiquitinated proteins through immunoprecipitation of proteins with $\mathrm{K}-\varepsilon-\mathrm{GG}$ recognizing antibodies (Xu \& Jaffrey, 2011, 2013; Xu et al., 2010). Once ubiquitinated peptides are purified as described above, LC-MS/MS analysis is performed and total ubiquitinated proteins are identified through searches against proteins of interest, in our case, the most recent update of the NCBI $A$. thaliana database.

Our ubiquitin remnant profiling was done through contract with Cell Signaling Technology (Danvers, MA) and was performed on total cell lysates collected from wild-type and nph3-6 seedings from two different treatment conditions, darkness and low intensity $\left(0.1 \mu \mathrm{mol} \mathrm{m}{ }^{-2} \mathrm{~s}^{-1}\right)$ blue light for four hours, in quadruplicate. It was found that blue light induced ubiquitination of phyA at two lysine residues, 555 and 603 (data not shown). 
This blue light-induced ubiquitination of phyA was not observed in the nph3-6 background, suggesting that this post-translational modification is dependent upon the CRL3 ${ }^{\mathrm{NPH} 3}$ complex (data not shown).

Given NPH3's central role in phot1-dependent phototropism and the evidence for phy $A$ and phot1 cooperation in blue light responses that include phototropism, these ubiquitin remnant profiling results were highly intriguing. In order to assess the role of $\mathrm{NPH} 3$ in phyA ubiquitination and the possibility that phy $\mathrm{A}$ ubiquitination could provide a link between phot1 and phyA, we used site (SDM) of phyA-GFP to convert lysine 555 and 603 to arginine, thus preventing ubiquitination at these lysines. After generating homozygous lines expressing the phyA-GFP SDM mutations (phyA-SDM), we performed physiological and biochemical evaluations.

\section{Materials and Methods}

\section{Plant Growth and Treatment Conditions}

Three-day-old dark-grown $A$. thaliana seedlings were used exclusively in this study. All mutant and arginine lines are in Columbia (Col-0) background. When possible, mutants and transgenic genotypes used had been previously described phot1-5 (Huala et al., 1997), phot1, phot2 (Kinoshita et al., 2001), (Sakamoto, 2002), phyA211 (Reed, Nagatani, Elich, Fagan, \& Chory, 1994), phyA-GFP (Toledo-Ortiz et al., 2010), and phyA overexpressor (Boylan \& Quail, 1991). Transgenic ubiquitin mutants were created by SDM of phyA clone (promoter phyA::PHYA-GFP) in pPZP211 that was obtained from A. Nagatani 
(Kyoto University). Throughout this study, three transgenic lines B2A, G2A, and I2D are used, each representing independent transformation events, that express

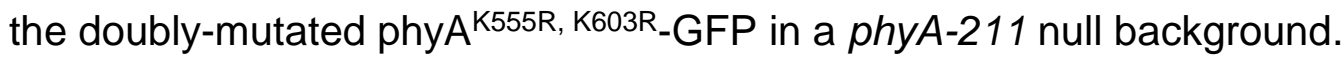
Seedlings were grown on half-strength Murashige and Skoog media with 1\% agar with or without filter paper depending upon the experiment as described below. Seeds were surface-sterilized using 30\% (v/v) commercial bleach for 30 min on an agitator and subsequently washed three times with $1 \mathrm{~mL}$ sterile water. Seeds were then either 1) plated on filter paper placed on the surface of growth medium in Petri plates if seedlings were to be collected for protein blotting, or 2) plated without filter paper using a thin layer of top-agar if seedlings were to be used for phototropic assays. Plated seeds were then cold treated at $4^{\circ} \mathrm{C}$ for $2-5$ days in darkness before treatment with white light for 1 hour to initiate uniform germination. The plates were then transferred to complete darkness and the seedlings allowed to grow for three days. All phototropic assays were conducted as described previously (Roberts et al., 2011). An alternative method of plating growing seedlings was used for hypocotyl elongation and growth inhibition assays. Sterilized seeds were placed in rows on medium in square plates and seedlings allowed to grow vertically along the surface of the medium. The entire plate was then scanned with a high-resolution scanner and images of seedlings measured using FIJI software (Schindelin et al., 2012; Schindelin, Rueden, Hiner, \& Eliceiri, 2015). Depending on the assay, either the straight-line tool (hypocotyl length) or the angle tool (hypocotyl curvature) tool were used in FIJI. 


\section{Light Treatments}

For phototropic assays seedlings were either mock-irradiated or exposed to unilateral blue light for different time-intervals and light intensities as described previously (Stowe-Evans et al., 1998). Low intensity blue light $\left(0.1 \mu \mathrm{mol} \mathrm{m}{ }^{-2} \mathrm{~s}^{-1}\right)$ was generated as described previously (Stowe-Evans et al., 1998). Very low intensity $\left(0.01 \mu \mathrm{mol} \mathrm{m}{ }^{-2} \mathrm{~s}^{-1}\right)$ blue light was generated as low-intensity blue light but with the addition of layers of cheesecloth to decrease light intensity further. High-intensity blue light $\left(100 \mu \mathrm{mol} \mathrm{m} \mathrm{m}^{-2} \mathrm{~s}^{-1}\right)$ was generated by filtering light from a Navitar Xenon 560 projector 550-W xenon lamp (Model LI-250) through a blue acrylic filter (Rohm and Haas No. 2424, 3.18-mm thick; Cope Plastics). Red and far-red-light treatments were generated via an LED bank built and designed for the purpose. For each color 10 LEDs were placed in a grid and connected in series with light output-intensity regulated by a potentiometer, allowing for an output range of approximately 5 to $50 \mu \mathrm{mol} \mathrm{m}^{-2} \mathrm{~s}^{-1}$ at a $25 \mathrm{~cm}$ distance from the light source. All materials to construct the LED bank were purchased through LED Supply (Lakewood, CO. The LEDs used in the construction of the light bank were: Red, Cree XLamp Photo Red High Power Star (single configuration); Blue, Cree XLamp Royal Blue High Power Star (single configuration); and Far-Red, Cree XLamp High Power Star (single configuration). The wavelength output of each LED was: red, $660 \mathrm{~nm} \pm 10$, blue, $450 \mathrm{~nm} \pm 10$, and far-red, $730 \mathrm{~nm} \pm 10$. In addition to the LED light-bank, seedlings were treated with far-red light by filtering white light from an 80 Watt Great Value ${ }^{\text {TM }}$ PAR38 Halogen Flood lightbulb 
through a Plexiglas ${ }^{\circledR}$ G 3143 infrared transmitting acrylic sheet purchased from www.eplastics.com (San Diego, CA). The acrylic sheet was placed beneath a tank (30 $\mathrm{cm}$ wide $\times 60 \mathrm{~cm}$ long $\times 12 \mathrm{~cm}$ deep) of infrared absorbing water and transmitted far-red light at $730 \mathrm{~nm}$. Red-light treatments for phototropic enhancement were performed from above by filtering white florescent light through a single sheet of red acrylic (Rohm and Haas 2444).

\section{Pharmacological treatments}

For experiments using a proteasome inhibitor treatment (Gladman, Marshall, Lee, \& Vierstra, 2016; D. H. Lee, 1998), seedlings were placed in a $1 \frac{1}{2}$ X MS liquid medium bath supplemented with either MG132 (Adipogen Life Sciences), bortezomib (Avachem Scientific) or solvent (DMSO) alone and gently oscillated for 2 hours prior to light-treatment or mock-irradiated.

\section{Protein Extraction}

Total protein was extracted from seedling tissue, which had been mock- or lighttreated with or without pharmacological agent. Seedlings were flash frozen in liquid nitrogen, and protein prepped immediately or stored at $-80^{\circ} \mathrm{C}$. Frozen tissue was ground with pre-chilled mortars and pestles in ice-chilled total extraction buffer (50mM HEPES, pH 7.8, 300 mM Sucrose, $150 \mathrm{mM} \mathrm{NaCl}_{2}, 10$ $\mathrm{mM}$ NaAcetate, $5 \mathrm{mM}$ EDTA, and 1X protease inhibitor cocktail [Sigma], 1mM NEM, and 1\% Triton X-100). All tissue and protein extract from grinding to electrophoretic analysis was performed in a green-lit $4^{\circ} \mathrm{C}$ cold-room. After grinding in extraction buffer, samples were allowed to rest on ice for 30 minutes 
before centrifugation at $10,000 \mathrm{~g}$ for 10 minutes to separate total protein from unprocessed material and particulate matter. Total protein samples were quantified using a Bradford assay (Toth, Ismaiel, \& Chen, 1999). Protein samples were either frozen for later use or loaded immediately onto a SDSpolyacrylamide gel using 6x-SDS Laemmli sample loading buffer.

\section{Running SDS-Polyacrylamide Gels and immunoblotting}

Proteins were separated by SDS-PAGE(8\% polyacrylamide) and transferred to nitrocellulose membranes by electroblotting for subsequent immunodetection. To immunodetect proteins, nitrocellulose membranes were initially blocked using $5 \%$ (w/v) powdered milk in $20 \mathrm{mM}$ Tris- $\mathrm{HCl}, \mathrm{pH} 7.6,137 \mathrm{mM} \mathrm{NaCl}$, and $0.05 \%$ Tween 20 (TBST) overnight at $4^{\circ} \mathrm{C}$ with rocking or for 2 hours at room temperature. After the first blocking, the membrane was incubated with an appropriate primary antibody for 1.5 hours at room temperature or overnight at $4^{\circ} \mathrm{C}$ in TBST plus $1 \%$ dry milk. After primary antibody treatment, membranes were washed 6-8 times with TBST for not less than an hour, followed by a second blocking in $1 \% \mathrm{w} / \mathrm{v}$ dry milk in TBST followed primary antibody wash for 30 minutes at room temperature or overnight with rocking. Membranes were then incubated with appropriate HRP-conjugated secondary antibody in TBST plus $1 \%$ dry milk for 1.5 hours at room temperature with rocking. Lastly, membranes were washed 6-8 times with TBST for no less than one hour, and proteins detected via chemiluminescence using HRP substrate (Millipore). 


\section{Antibodies}

The anti-NPH3 antibodies have been described previously (Motchoulski \& Liscum, 1999). Other antibodies used include: Anti-GFP PAB1264 (Abnova), Anti-phyA, 073D ascites (generously provided by P. Quail lab; PGEC, Berkley, CA), goat-anti-rabbit-HRP (Genescript A00098), goat-anti-mouse-HRP (Bio-Rad 170-6516), agarose-conjugated anti-GFP (MBL International), and anti-ubiquitin P4D1 (Cell Signaling Technology).

\section{Immunoprecipitations}

Immunoprecipitation (IP) of phyA-GFP was performed on total protein extract using agarose-conjugated anti-GFP beads (MBL International). In brief, anti-GFP agarose beads were resuspended in total grinding buffer by washing the beads three times in $200 \mu \mathrm{l}$ total grind buffer. After washing beads, total protein extract was added to the beads; $200 \mu \mathrm{g}$ total protein was added to each IP tube, and total volume equalized by adding total grind buffer to each tube to equal volume. Additionally, $1 \mu \mathrm{l}$ of PIC (protease inhibitor cocktail [Sigma]) was added for each $100 \mu \mathrm{g}$ of protein added. The IP beads-protein samples were placed in a $4^{\circ} \mathrm{C}$ rotator for four hours, then rinsed three times in $500 \mu$ total. Finally, $1 \times$ SDS loading buffer was added, and the samples were boiled for $5 \mathrm{~min}$, pulsed in a centrifuge to pellet beads, and loaded onto a polyacrylamide gel. 


\section{Results and discussion}

\section{Transgenic lines expressing phy $\mathrm{A}^{\mathrm{K} 555 \mathrm{R}, \mathrm{K} 603 \mathrm{R}}$-GFP exhibit normal}

red light-induced phyA degradation and far-red light-induced

\section{growth inhibition}

Three transgenic lines (B2A, G2A, I2D), representing independent transformation events, that express the doubly-mutated phy ${ }^{\mathrm{K} 555 \mathrm{R}, \mathrm{K} 603 \mathrm{R}}$-GFP in a phyA-211 null background exhibit normal red light-induced phyA degradation (Fig. 2.1).

Not surprisingly, given the normal turnover of the mutant phyA we found that the mutant transgenic lines exhibited a normal high fluence rate dependent hypocotyl inhibition response in far-red light (fig.2.2 A and B). These results confirmed that the phy $A^{\mathrm{K} 555 \mathrm{R}, \mathrm{K} 603 \mathrm{R}}$ protein is functional for these classic phyA light responses, and that as such any attenuation we observe for blue light-induced responses is specific for the phy $A^{\mathrm{K} 555 \mathrm{R}, \mathrm{K} 603 \mathrm{R}}$ mutants and not a general disfunction of the mutant phyA. 


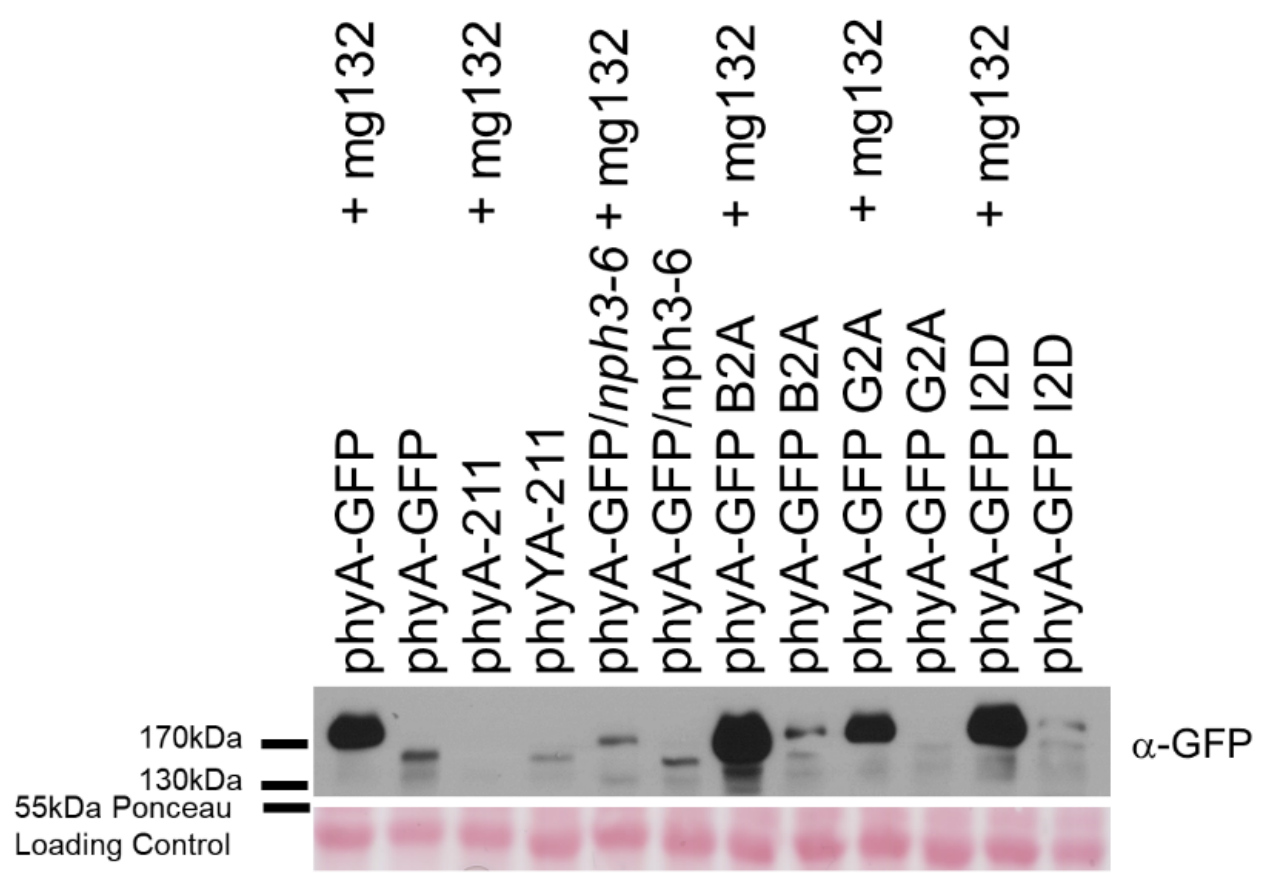

Figure 2.1 Red Light-induced degradation of phyA is not altered in phyA ${ }^{\mathrm{k5} 55 \mathrm{R}, \mathrm{K} 603 \mathrm{R}}$ lines Immunoblot analysis of phyA from total extracts treated with $50 \mu \mathrm{mol} \mathrm{m}{ }^{-2} \mathrm{~s}^{-1}$ red light from above with or without $50 \mu \mathrm{M}$ MG132. Bottom ponceau stain of blot as loading control. 
A)

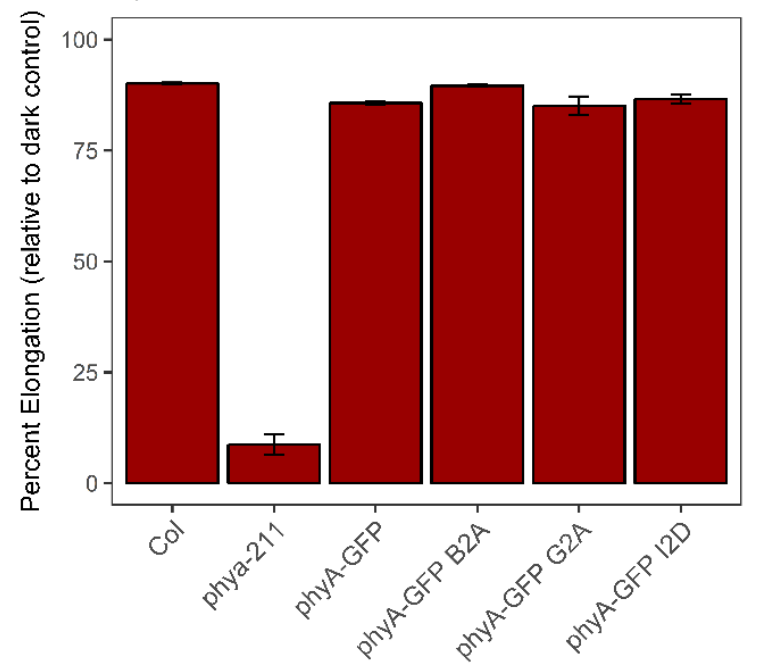

B)

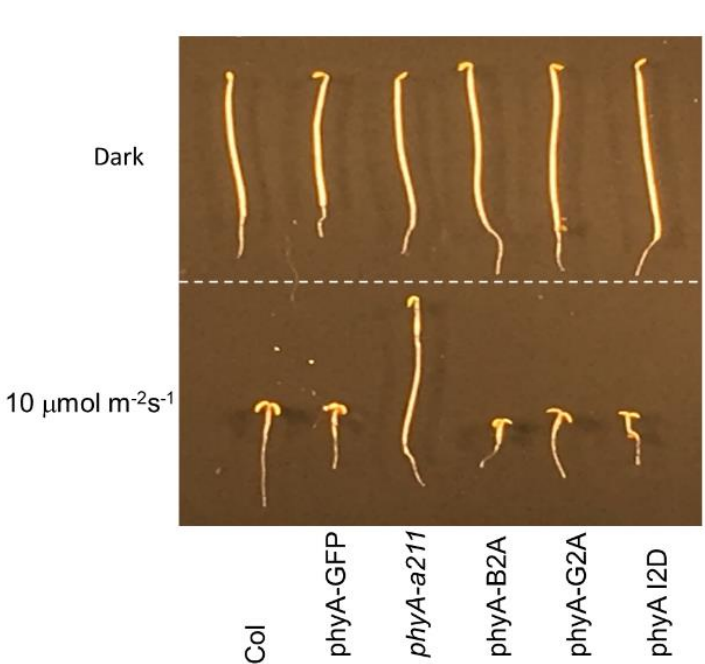

Figure 2.2 Far-red light-induced hypocotyl grownth inhibition in phyA ${ }^{\mathrm{K} 555 \mathrm{R}, \mathrm{K} 603 \mathrm{R}}$ lines is not different from phyA-GFP or Col.

A, percent elongation after 3-day treatment of $10 \mu \mathrm{mol} \mathrm{m}^{-2} \mathrm{~s}^{-1}$ far-red light relative to dark-grown seedlings. B, representative seedlings grown for $3 d$ in darkness or far-red light illustrating the extent of far-red-induced hypocotyl inhibition. Data represent mean responses \pm SE for a minimum 90 seedlings of 3 replicate experiments. 


\section{phyA ${ }^{555 R, ~ K 603 R ~ l i n e s ~ e x h i b i t ~ n o r m a l ~ p h o t o t r o p i s m ~ i n ~ l o w ~ a n d ~}$}

\section{very low fluence blue light}

As previously described, phyA is necessary for the phototropic response in low

$\left(0.1 \mu \mathrm{mol} \mathrm{m} \mathrm{m}^{-2} \mathrm{~s}^{-1}\right)$ and very low $\left(0.01 \mu \mathrm{mol} \mathrm{m}^{-2} \mathrm{~s}^{-1}\right)$ blue light conditions (C. W. Whippo \& Hangarter, 2004). All three transgenic lines demonstrated similar phototropic curvature patterns to the phyA-GFP transgenic control and to Col-0 after a four-hour irradiation treatment of either low $\left(0.1 \mu \mathrm{mol} \mathrm{m}^{-2} \mathrm{~s}^{-1}\right)(\mathrm{fig} .2 .3 \mathrm{~A})$ or very low $\left(0.01 \mu \mathrm{mol} \mathrm{m}{ }^{-2} \mathrm{~s}^{-1}\right)$ (fig $\left..2 .3 \mathrm{~B}\right)$ unilateral blue light, indicating that phy $A^{K 555 R, K 603 R}$ is able to function normally in low and very low fluence rate blue light phototropic responses.

Given the fact that phyA is required for phototropism under low and very low intensity blue light conditions, and that phyA ubiquitin remnant profiling indicated phy $A$ is being ubiquitinated under low intensity blue light conditions, we predicted that phy $A^{K 555 R, K 603 R}$ expressing lines would have reduced or null phototropic phenotypes similar to curvature seen in seedlings harboring the phyA-211 null mutations. Our low and very low phototropism data (fig. 2.3 A and B) suggests that either the lysine residues targeted in phyA blue light-dependent CRL3 ${ }^{\mathrm{NPH} 3}$ dependent ubiquitination are not necessary for phyA-dependent modulation of low and vary low fluence rate phototropism, or that alternate sites are being ubiquitinated such that ubiquitination-dependent function is maintained despite site directed mutagenesis. 
A)

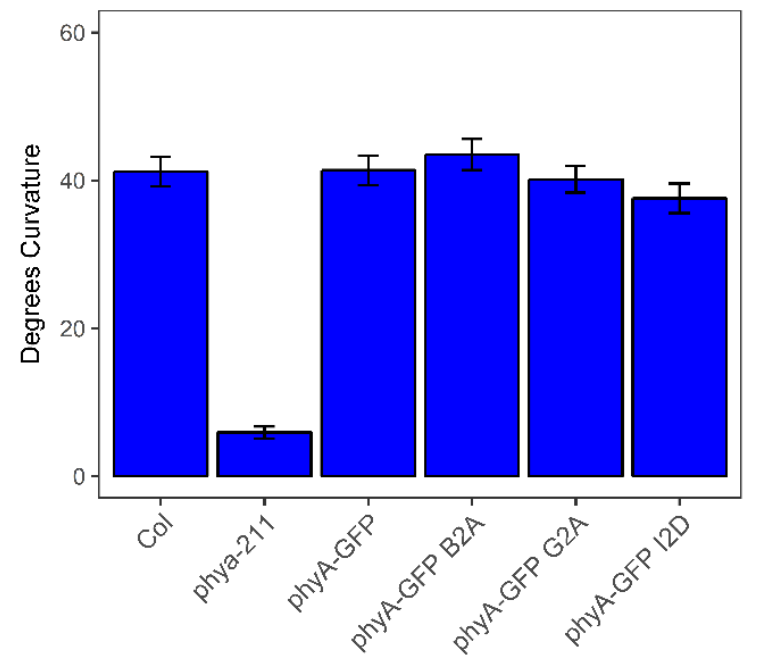

B)

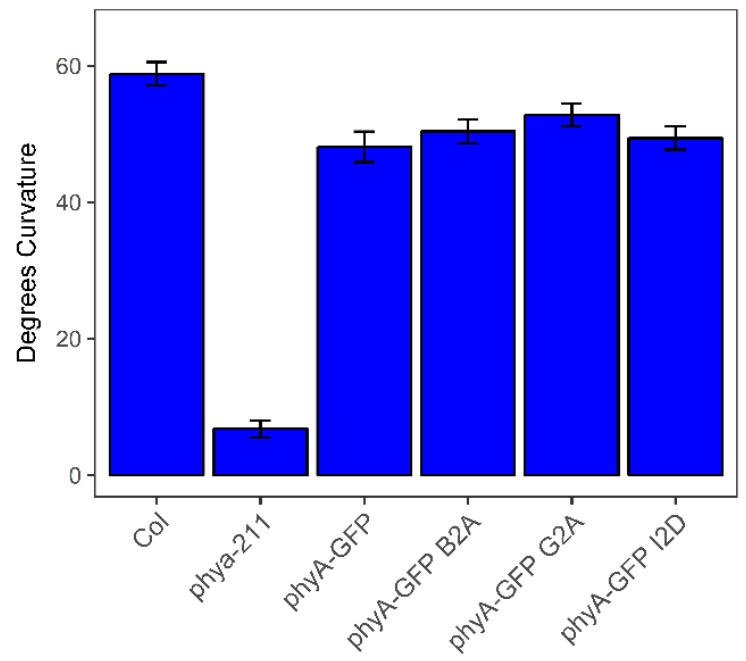

Figure 2.3 phyA $^{\mathrm{K} 555 \mathrm{R}, \mathrm{K} 603 \mathrm{R}}$ lines respond phototropically normal to low and very-low fluence rate blue light

Three-day-old etiolated $A$. thaliana seedlings were treated with low (A) $0.1 \mu \mathrm{mol} \mathrm{m}^{-2} \mathrm{~s}^{-1}$ or very low (B) 0.01 $\mu \mathrm{mol} \mathrm{m} \mathrm{m}^{-2} \mathrm{~s}^{-1}$ unilateral blue light for 4 hours, and phototropic curvature determined. Neither low $(A)$ or very low (B) unilateral blue light treated phy $A^{\mathrm{K} 555 \mathrm{R}, \mathrm{K} 603 \mathrm{R}}$ seedlings exhibited prototrophic responses different from phyA-GFP and Col-0 controls. In both low (A) and very low (B) unilateral blue light, phyA-211 showed a near phototropic null phenotype. Data represent mean responses \pm SE for a minimum 90 seedlings of 3 replicate experiments. 


\section{phyA $^{\text {K555R, K603R_GFP lines do not exhibit red light-induced }}$}

\section{phototropic enhancement}

Red light pre-irradiation treatments are known to result in an enhancement to phototropic curvature, a response that is also phyA-dependent (Emily L. StoweEvans, Darron R. Luesse et al., 2001; Parks et al., 1996). The red light-induced phototropic enhancement response is an instance of red light-mediated modulation of blue light-induced phototropism. We predicted that phyA-SDM lines would not function in red light phototropic enhancement responses. We assessed red light-induced phototropic enhancement by performing a phototropic assay with and without a pretreatment with red light $\left(160 \mu \mathrm{mol} \mathrm{m}{ }^{-2}\right)$, which previous studies had shown to enhance the bending response (Emily L. StoweEvans, Darron R. Luesse et al., 2001). The result of our phototropic assay is somewhat difficult to interpret because no red light-induced phototropic enhancement was observed in transgenic control carrying the wild-type phyAGFP (Fig. 2.4). A similar lack of phototropic enhancement was observed in

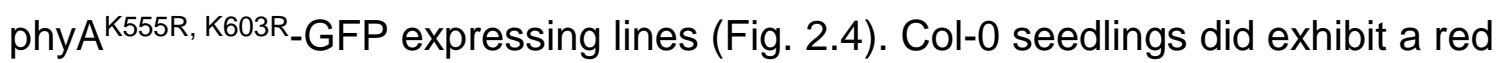
light enhancement response (Fig. 2.4), indicating that the assay itself worked and seedlings saw levels of red and blue light that induces phototropic enhancement in the wild-type background.

The lack of phototropic enhancement may reflect a dysfunction of phyA in both control phyA-GF and phyA ${ }^{\mathrm{K} 555 \mathrm{R}, \mathrm{K} 603 \mathrm{R}}$ lines because of the GFP tag. Further experiments with transgenics lacking the GFP tag would address this hypothesis. 


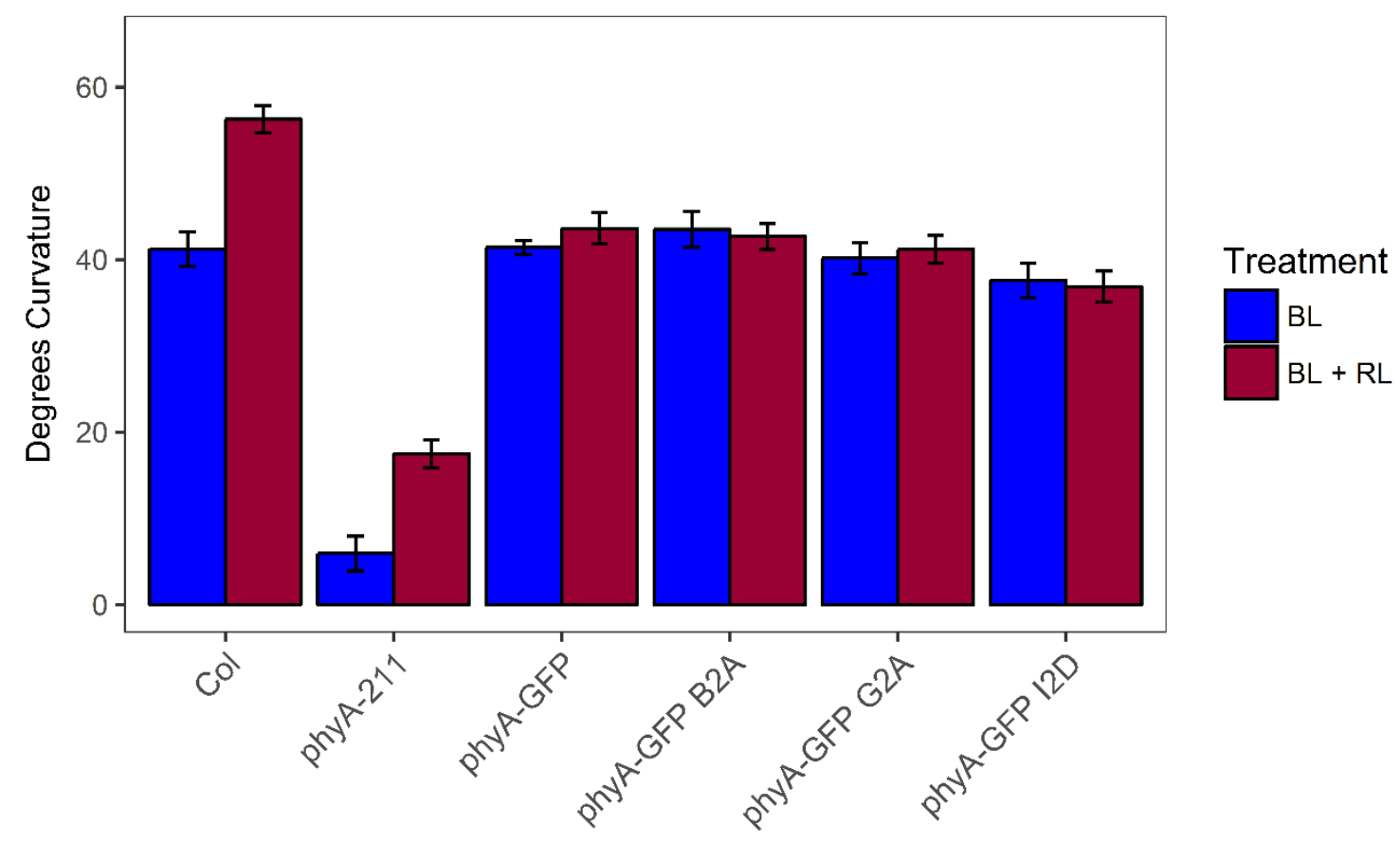

Figure 2.4 phyA ${ }^{\mathrm{K} 555 \mathrm{R}, \mathrm{K} 603 \mathrm{R}}$ lines did not exhibit red light-enhancement of phototropic curvature in low fluence blue light

Three-day-old etiolate seedlings were treated with $0.1 \mu \mathrm{mol} \mathrm{m} \mathrm{m}^{-2} \mathrm{~s}^{-1}$ unilateral blue light or $0.1 \mu \mathrm{mol} \mathrm{m} \mathrm{m}^{-2} \mathrm{~s}^{-1}$ unilateral blue light for four hours plus a $160 \mu \mathrm{mol}$ pulse of red light from above at the start of the blue light treatment before being collected on transparency film and having their hypocotyls traced. Data represent mean responses $\pm \mathrm{SE}$ for a minimum 90 seedlings of 3 replicate experiments. 


\section{phy ${ }^{\mathrm{K} 555 \mathrm{R}, \mathrm{K} 603 \mathrm{R}}$ lines have reduced phototropic curvature in high intensity blue light}

It has been reported that phyA attenuates the phototropic response under conditions of very high intensity blue light $\left(100 \mu \mathrm{mol} \mathrm{m} \mathrm{m}^{-2} \mathrm{~s}^{-1}\right)$; seedlings lacking a functional copy of phyA display greater curvature than wild-type seedlings (C. W. Whippo \& Hangarter, 2004). We therefore hypothesized that the blue lightinduced ubiquitination might impact the high intensity blue light phototropic attenuation response. Though we did not observe enhanced bending in the phya211 null mutant under the conditions we assayed phototropism under very highintensity blue light, we did observe dramatically reduced phototropism in each of the phyA ${ }^{\mathrm{K} 555 R, \mathrm{~K} 603 \mathrm{R}}$ lines (Fig. 2.5). In fact, phototropic responsiveness of the phy $A^{K 555 R, K 603 R}$ lines was clearly diverged by 4 hours after the start of blue light exposure, and remained for the duration of the experimental protocol (Fig. 2.5). At first these results may seem counterintuitive, but if phyA causes phototropic signal attenuation, retention of phyA by modification to its ubiquitination sites, preventing its degradation, could result in the opposite phenotype to that observed in seedlings where phyA is absent. 


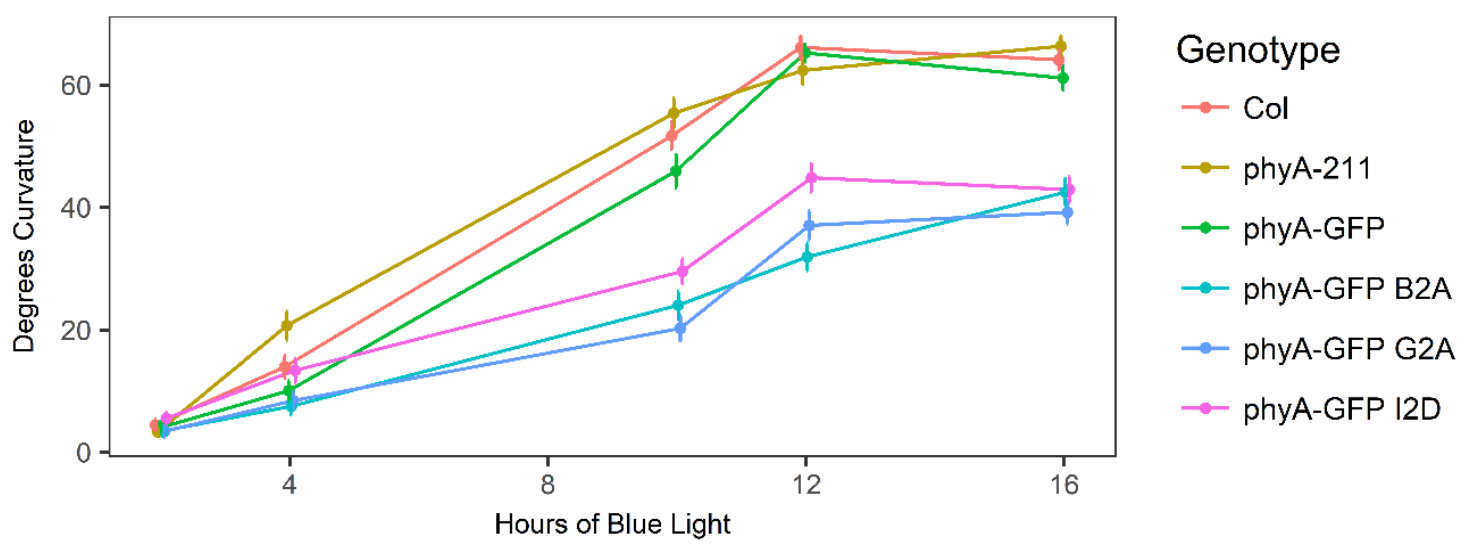

Figure 2.5 phy $\mathrm{A}^{\mathrm{K} 555 \mathrm{R}, \mathrm{K} 603 \mathrm{R}}$ lines responded prototropically at reduced levels to high intensity blue light-treatments compared to Col-0, phyA-GFP, and phyA-211

Three-day-old etiolated seedlings treated with $100 \mu \mathrm{mol} \mathrm{m} \mathrm{m}^{-2} \mathrm{~s}^{-1}$ unilateral blue light for 2 to 16 hours. After light-treatment, seedlings were collected on transparency film and their hypocotyls were traced. Data represent mean responses \pm SE for a minimum 90 seedlings of 3 replicate experiments. 


\section{Altered high intensity blue light-induced phototropism in

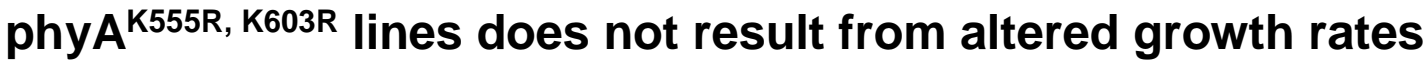

A hypocotyl elongation assay was performed over a 4-days on etiolated seedlings. As shown in figure 2.6, although seedling lengths varied across genotypes at any given timepoint, the rates of elongation (shape of curves) were very similar for each genotype. These data suggest that any differences in

phototropic curvatures between controls and the phy $A^{K 555 R, K 603 R}$ lines in high light conditions were not due to differences in rate of hypocotyl elongation. 


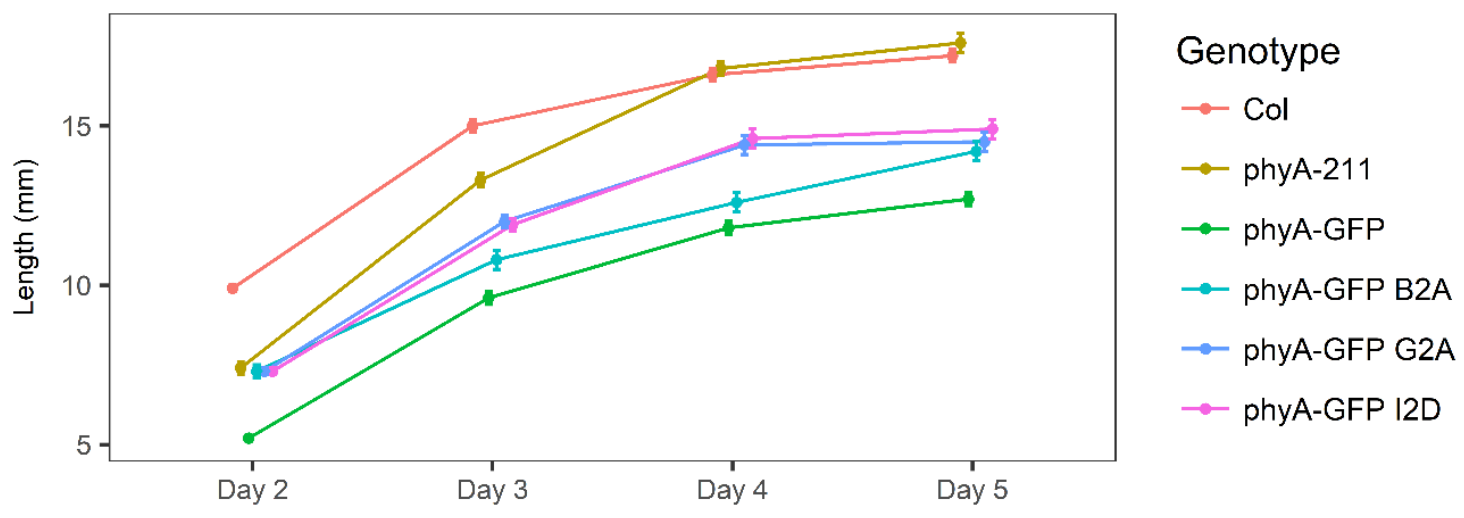

Figure 2.6 phy $\mathrm{A}^{\mathrm{K} 555 \mathrm{R}, \mathrm{K} 603 \mathrm{R}}$ lines are functional in hypocotyl elongation

Seedlings were grown in darkness and samples taken at indicated times to measure hypocotyl lengths as described in the methods. Data represent mean responses \pm SE for a minimum 90 seedlings of 3 replicate experiments. 


\section{phy $^{\mathrm{K} 555 \mathrm{R}, \mathrm{K} 603 \mathrm{R}}$ lines have reduced phyA turnover in high intensity blue light}

As mentioned earlier, ubiquitin remnant profiling indicated that phyA is ubiquitinated in lysine residues 555 and 603 in blue light in a CRL3 ${ }^{\mathrm{NPH}}{ }_{-}$ dependent manner, potentially marking it for degradation. We hypothesized that altering these ubiquitination sites in phyA would reduce ubiquitination levels and therefore decrease rate of proteasome-facilitated turnover in blue light conditions. We tested this hypothesis by treating seedlings with very high intensity blue light for 16 hours (conditions where we observed maximal phonotype affect [Fig. 2.6]) and assaying for phyA abundance in total protein samples by immunoblot analysis (Fig. 2.7 A). Comparing dark protein levels to blue light-treated levels provides a basis to measure turnover. Results indicate that phy ${ }^{\mathrm{K} 555 \mathrm{R}, \mathrm{K} 603 \mathrm{R}}$ in our transgenic lines is not degraded to the extent that wild-type phyA-GFP is in high blue light (Fig. 2.7 A), a finding consistent with very high intensity blue light phototropic data discussed earlier (Fig. 2.6). Interestingly, the pattern of wild-type phyA-GFP degradation over time in high intensity blue light (Fig. 2.7 B) parallels the change in phototropic responsiveness in high blue light (Fig. 2.6); the stronger the degradation the stronger the phototropic response.

Although our phy $\mathrm{A}^{\mathrm{K} 555 \mathrm{R}, \mathrm{K} 603 \mathrm{R}}$ lines exhibit reduced degradation in high blue light, phy ${ }^{K 555 R, K 603 R}$ is not fully stabilized. There are several explanations for why phy $A^{K 555 R, K 603 R}$ is only partially stabilized. First, ubiquitin remnant profiling identified six lysine residues in phyA that were ubiquitinated in response to blue 
light exposure (K555, K588, K598, K603, K942, and K946) but only K555 and K603 exhibited NPH3 dependency. Because we are primarily interested in ubiquitination events that are dependent upon $\mathrm{NPH} 3$, only these sites have been analyzed. It is probable that one or more of the remaining four site identified as blue light-responsive could mark phyA for degradation. It is also possible that the ubiquitination machinery may be utilizing alternative lysine residues as sites for ubiquitination in the phy $\mathrm{A}^{\mathrm{K} 555 \mathrm{R}, \mathrm{K} 603 \mathrm{R}}$ lines. Ubiquitin ligases are known to be promiscuous where mutation at one ubiquitination site results in protein ubiquitination at another nearby sites (Chan, 2006; Tsz, Yam, \& Poon, 2005). Though these possibilities have not yet been tested, the evidence that phy ${ }^{\mathrm{K} 555 \mathrm{R} \text {, }}$ K603R lines have reduced phototropic curvature and partially stabilized phyA levels relative to the phyA-GFP control is consistent with lysine residues 555 and 603 being important for phyA turnover in blue light and regulation of phototropism. To evaluate if ubiquitination of phyA is altered in the phy $A^{\mathrm{K} 555 \mathrm{R}, \mathrm{K} 603 \mathrm{R}}$ lines, we wished to use methods similar to those used to detect ubiquitination levels of phot1 (Roberts et al., 2011). In brief, we immunoprecipitated from total protein samples collected from either dark-grown seedlings or seedlings treated with both 16 hours $100 \mu \mathrm{mol} \mathrm{m} \mathrm{m}^{-2} \mathrm{~s}^{-1}$ unilateral blue light, followed by immunodetecting with anti-ubiquitin antibodies. However, because phyA is degraded in response to high intensity blue light, we first needed to be able to pharmacologically stabilize the protein in order to detect phyA ubiquitination. Although we could stabilize phyA in red light-treated plants by pre-treatment with MG132, we were unable to stabilize phyA in blue light with MG132 (data not shown). We did find 
that another proteasome inhibitor with higher potency, bortezomib (Btz) at concentrations of $100 \mu \mathrm{M}$ or greater could stabilize a fair proportion of phyA under high intensity blue light (Fig. 2.7 C and D) (Gladman et al., 2016). Therefore, we performed immunoprecipitation and subsequent immunoblot analysis for ubiquitination in seedlings mock-irradiated or irradiated with $16 \mathrm{hrs}$. of high intensity $\left(100 \mu \mathrm{mol} \mathrm{m}{ }^{-2} \mathrm{~s}^{-1}\right)$ blue light and $100 \mu \mathrm{M} \mathrm{Btz}$. As shown in figure 2.7 $\mathrm{E}$, there is no detectable phyA ubiquitination in samples from dark-grown seedlings, but there is clear ubiquitination of phyA in samples from blue lighttreated seedlings. Moreover, there is no obvious difference in the ubiquitination level between control phyA-GFP plants and the phyA ${ }^{\mathrm{K} 555 \mathrm{R}, \mathrm{K} 603 \mathrm{R}}$ lines (Fig. $2.7 \mathrm{E}$ ). These results indicate that phyA in the phy ${ }^{\mathrm{K} 555 R, \mathrm{~K} 603 \mathrm{R}}$ lines is indeed still ubiquitinated (Fig. 2.7 E) consistent with explanations offered above about why phy $A^{\mathrm{K} 555 \mathrm{R}, \mathrm{K} 603 \mathrm{R}}$ is only partially stabilized (Fig. 2.7 A). However, lack of ubiquitination at lysine residues 555 and 603 , although we cannot specifically detect these alterations with our present immunoblot methods, does appear to influence the targeting of phyA to the proteasome to some extent (Fig. 2.7 A). 
A)

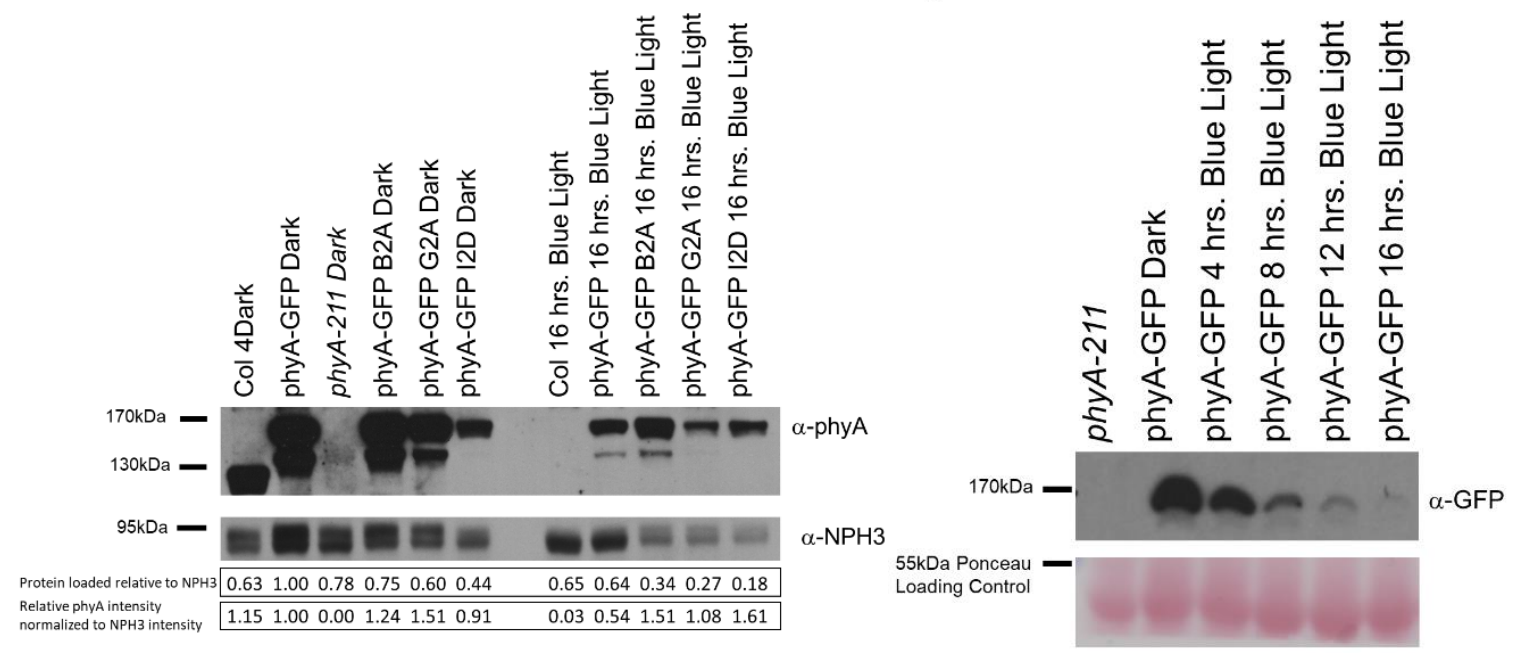

C)
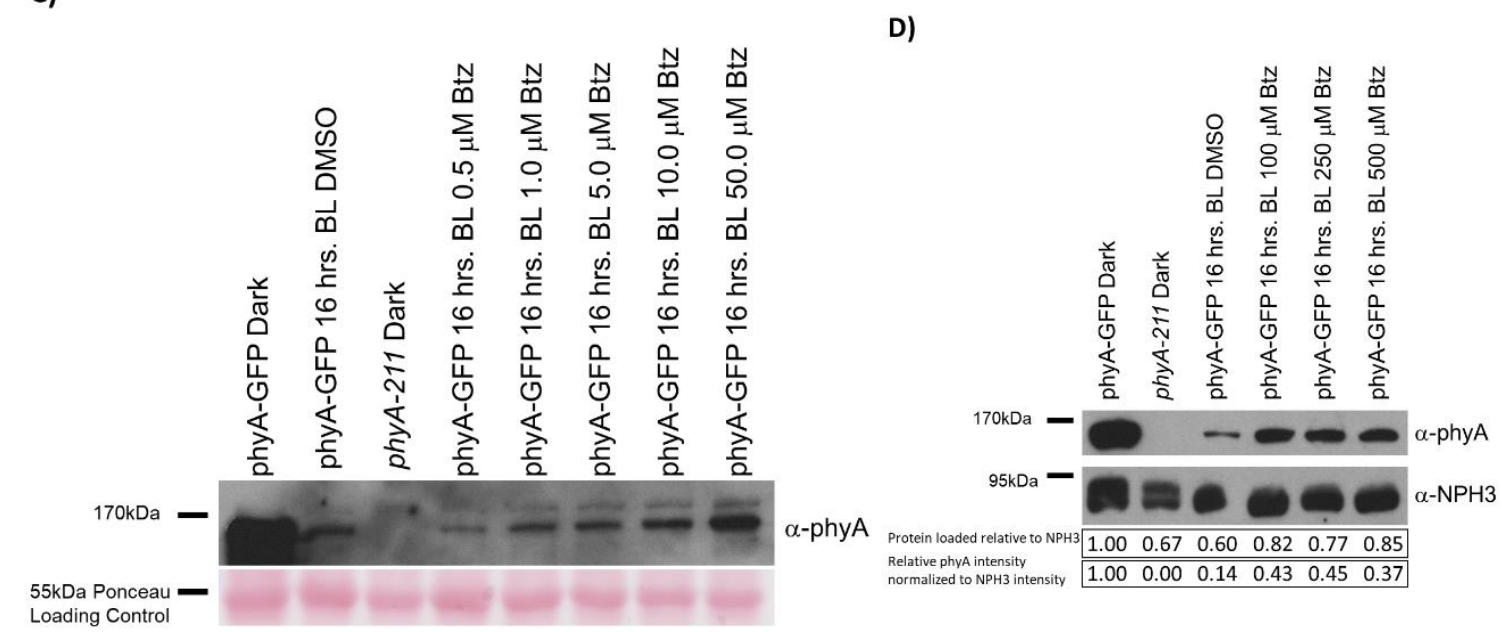

E)

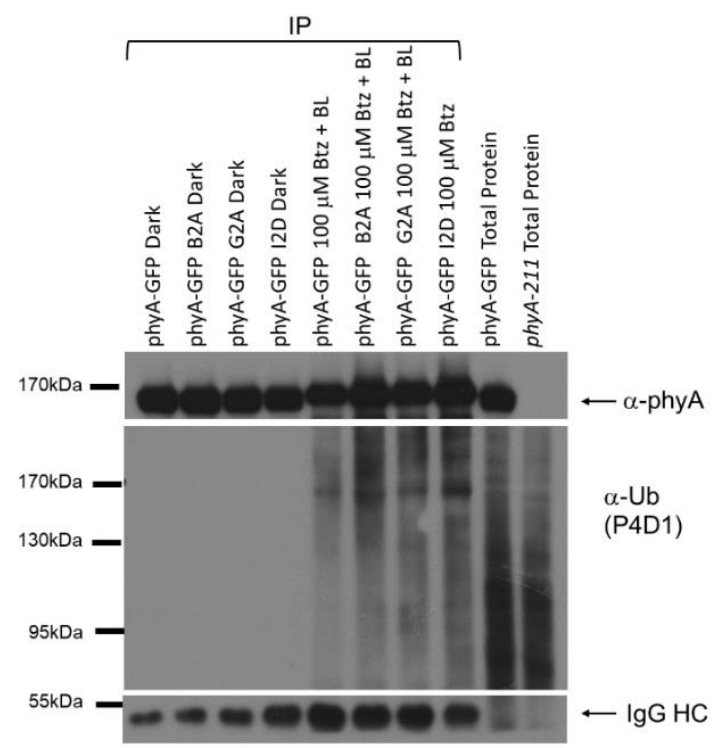


Figure 2.7 phyA $^{\mathrm{K} 555 \mathrm{R}, \mathrm{K603R}}$ lines display reduced phyA degradation in high blue light

A, Immunoblot analysis of phyA abundance in in 16 hours high intensity $\left(100 \mu \mathrm{mol} \mathrm{m} \mathrm{m}^{-2} \mathrm{~s}^{-1}\right)$ unilateral blue light. Top blot was probed with anti-phyA antibodies; bottom blot was probed with anti-NPH3 antibodies (as a loading control). First numeric panel shows the relative intensity of NPH3 loading control bands. Second numeric panel shows the relative abundance of phyA normalized to phyA-GFP dark-grown protein levels adjusted for differences seen in NPH3 loading control. B, immunoblot time course analysis of phyA-GFP abundance in total proteins from seedlings treated with high intensity $\left(100 \mu \mathrm{mol} \mathrm{m} \mathrm{m}^{-2} \mathrm{~s}^{-1}\right)$ unilateral blue light. Ponceau staining is shown as a loading control. C and D, immunoblot analysis of phyA in total protein from seedlings treated with high intensity $\left(100 \mu \mathrm{mol} \mathrm{m} \mathrm{m}^{-2} \mathrm{~s}^{-1}\right)$ unilateral blue light and the proteasome inhibitor bortezomib (Btz). For panel $\mathrm{C}$ a ponceau stain is shown as a loading control, while for panel $\mathrm{D}$ a blot probed with anti-NPH3 antibodies is shown as a loading control. E, immunoblot of phyA and phyA-Ub in immunoprecipitants from total protein from dark-grown and high intensity blue light-treated seedlings. Total protein samples from the phyA-GFP and phyA-211 null-mutant are shown as positive controls for ubiquitination. Top blot was probed with anti-phyA antibodies, middle blot was probed with anti-ubiquitin antibodies, P4D1, that recognize both mono-and poly-ubiquitination. The bottom panel shows the IgG band resultant from the immunoprecipitation as a loading control. 


\section{Overexpression of oat phyA in A. thaliana does not phenocopy the phy $A^{K 555 R, K 603 R}$ lines for phototropism in high intensity blue \\ light}

Because we observed a direct correlation between abundance of phyA in the phy $A^{K 555 R, ~ K 603 R ~ l i n e s ~ w i t h ~ p h o t o t r o p i c ~ r e s p o n s i v e n e s s ~ i n ~ h i g h ~ i n t e n s i t y ~ l i g h t ~(F i g . ~}$

2.5) we reasoned that we might observe a similar decrease in phototropism in lines where phyA levels are artificially increased through overexpression. However, as shown in figure 2.9 transgenic $A$. thaliana seedlings overexpressing an oat phy $(A O X)$ are indistinguishable prototropically from control wild-type and At.phyA-GFP lines. The AOX lines do indeed retain much of the phyA after blue light treatment that is observed in dark-grown plants (Fig. 2.9). Previous studies of red light-induced phot1-dependent phototropism in the AOX line have also shown no influence of this heterologous oat phyA in phototropism (Emily L. Stowe-Evans, Darron R. Luesse et al., 2001), despite the many phyA responses in which the transgenically expressed protein does appear to function (Boylan \& Quail, 1991; Jeong-il Kim et al., 2004; Kneissl, Shinomura, Furuya, \& Bolle, 2008). 


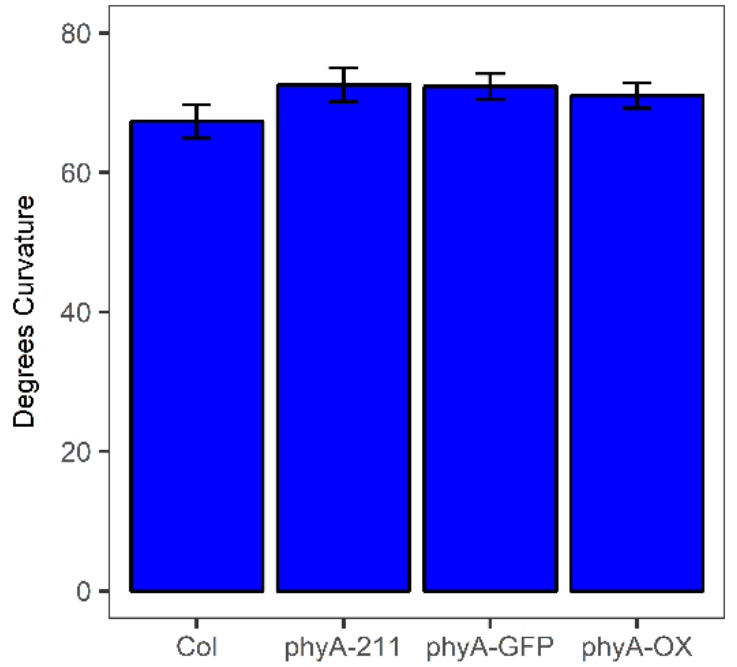

Figure 2.8 Transgenic $A$. thaliana lines over-expressing oat phyA do not exhibit altered phototropism in response to high intensity blue light

A. thaliana seedlings over-expressing oat phyA exhibit prototropically induced curvature like wild-type controls in high intensity $\left(100 \mu \mathrm{mol} \mathrm{m} \mathrm{m}^{-2} \mathrm{~s}^{-1}\right)$ blue light. Data represent mean responses $\pm \mathrm{SE}$ for a minimum 90 seedlings of 3 replicate experiments. 


\section{phot1, phot2, nor NPH3 appear to influence the turnover of phyA}

\section{in high intensity blue light}

Our ubiquitin remnant profiling indicated that phyA was ubiquitinated at lysine residues 555 and 603 in blue light in a NPH3-dependent manner. Because we have observed partial stabilization of phyA in high intensity blue light by mutation of target lysines (Fig. 2.7 A), a stabilization that is correlated with a defect in high intensity blue light-induced phototropism (Fig. 2.5), we wished to determine if $\mathrm{NPH} 3$ is required for this stabilization.

We conducted an immunoblot analysis of phyA abundance in total protein Col-0 and nph3-6 null mutants to test this. The results indicate that presence of NPH3 is not required for turnover of phyA in high intensity blue light; phyA in a nph3-6 background was degraded just as effectively as in a Col-0 background (Fig. 2.9). This result may suggest that phyA ubiquitination at lysines 555 and 603 may be facilitated by another or several other NRL family members. It should also be pointed out that the ubiquitin remnant profiling was performed on seedlings that had been treated with 4 hours $0.1 \mu \mathrm{mol} \mathrm{m} \mathrm{m}^{-2} \mathrm{~s}^{-1}$ blue light and treatment conditions for western blot analyses in this study were $100 \mu \mathrm{mol} \mathrm{m} \mathrm{m}^{-2} \mathrm{~s}^{-1}$ blue light for sixteen hours, 1000 times the light intensity for 4 times as long. Therefore, the action of another NRL member is highly likely. Alternatively, it is possible that phyA is ubiquitinated NPH3-dependently in low blue light at lysine residues 555 and 603 but another non-NRL ubiquitin ligase substrate adapter facilitates phyA ubiquitination under high intensity blue light conditions. This study did not 
experimently address NPH3-dependent low blue-light phyA stability in low blue light because we observed no obvious physiological phenotypes under those conditions.

We also investigated whether the phots themselves were necessacry for the high intenisty blue light-induced degradation of phyA by evaluating phyA turnover in several additional mutants: a phot1 null (phot1-5), and in a phot double null mutant, (phot1-5/phot2-1). Results demonstrate that phyA is degraded in both mutant backgrounds as in Col-0, indicating that neither phot1 or phot2 are necessicary for high intensity blue light-induced degredation of phyA. It seems probable the phyA serves as the blue light-recepter for its own degradation, a possibility that could be tested by examining blue light-induced degradation in seeedlings that lack light-sensing capacity, such as chromophore-deficient mutants (Takayuki Kohchi, Keiko Mukougawa, Nicole Frankenberg, Munehisa Masuda, Akiho Yokota, 2001; Takuya Muramoto, Takayuki Kohchi, Akiho Yokota, Inhwan Hwang, 1999). 


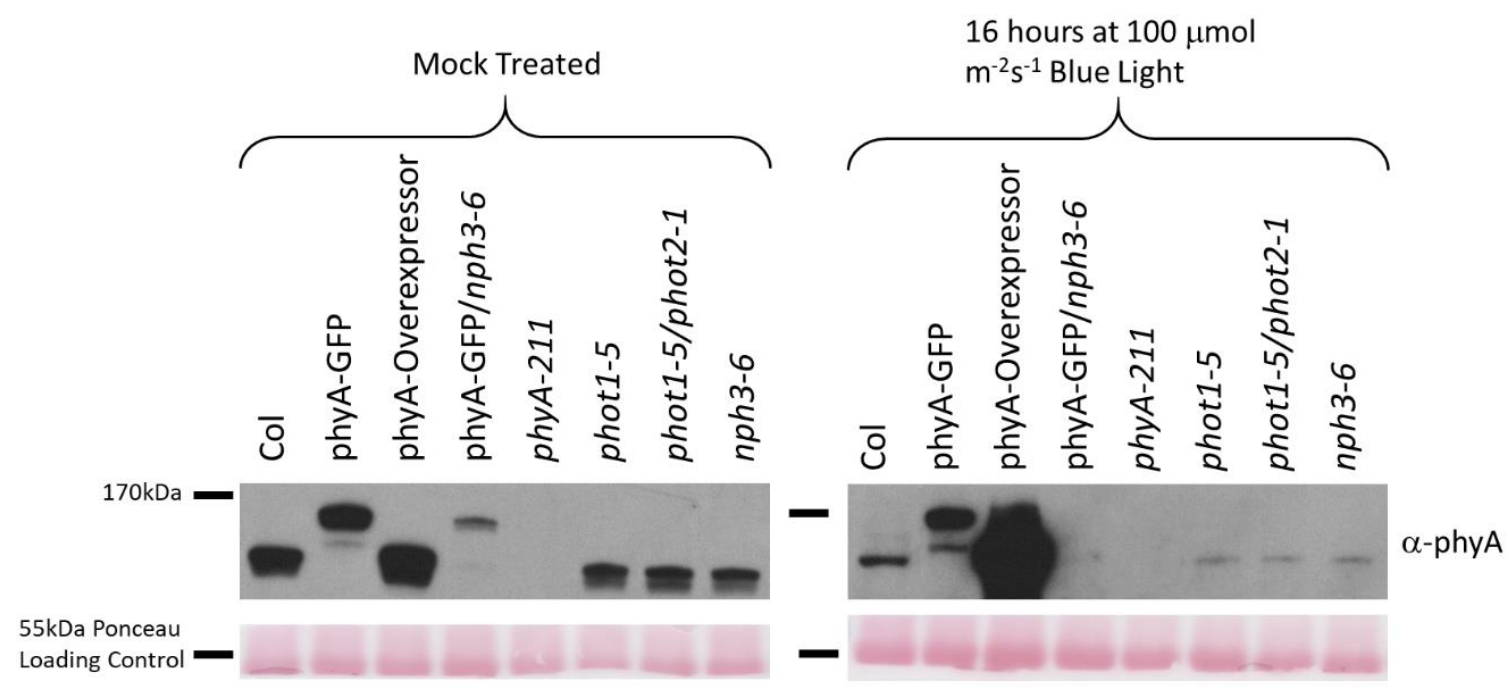

Figure 2.9 phot1, phot2, nor NPH3 modulate phyA degradation in high fluence blue light Immunoblot detection of phyA levels indicates that phyA levels are decreased after 16 hour treatments of $100 \mu \mathrm{mol} \mathrm{m}{ }^{-2} \mathrm{~s}^{-1}$ unilateral blue light in phot1-5, phot2-1, and nph3-6 backgrounds to the same extent as in Col background. 


\section{Chapter 3 COMPARISON OF LIGHT-REGULATED DEVELOPMENT DURING SEEDLING ESTABLISHMENT IN GLYCINE MAX AND GLYCINE SOJA.}

\section{Abstract}

\section{Background:}

Photomorphogenesis impacts development more than any other environmental stimuli and is critical for survival in establishing seedlings. Domestication events potentially eliminate genes and alter photomorphogenic responses valuable to crop species. Light is particularly important to seedlings during establishment and the transition from subterranean to aerial growth.

\section{Objective}

To characterize light responses in developing seedlings and differences in $G$. max and G. soja responsiveness.

\section{Method}

Glycine max and Glycine soja seedlings were grown in white light; red, blue, and far-red monochromatic light; and in darkness. Seedling development was analyzed by multiple photomorphological development parameters including: growth of roots, hypocotyl, first internode, second internode; total stem length; relative growth inhibition or stimulation to darkness; and percentage of seedlings reaching particular developmental stages.

\section{Results}


G. max and G. soja do not undergo the same photomorphogenesis in all light conditions. G. max generally invests in aerial development sooner than G. soja. G. soja is highly responsive to light in root elongation, while G. max is relatively unresponsive.

\section{Conclusion}

Domesticated G. max has multiple photomorphogenic responses that differ greatly from G. soja, suggesting that domestication has been highly impactful in shaping G. max and producing physiologically distinct photomorphogenic patterning relative to G. soja.

Keywords: Glycine max, Glycine soja, photomorphogenesis, seedling establishment, light responses, domestication, natural variation

\section{Introduction}

Plants are sessile, therefore, their perception and response to ever-changing environmental conditions is of paramount importance for their survival. Due to the autotropic nature of plants, it is perhaps not surprising that plants are highly adapted to detect cues associated with their light environment and perform physiological modulation as a means of maximizing their adaptation. Light is not only the source from which plants derive their energy, but also an invaluable source of information which enables plants to "see" the world around them: their proximity to other plants, the direction of light, the quality of light, and time. (Casal, 2013; Franklin \& Whitelam, 2005; Hohm, Preuten, \& Fankhauser, 2013;

Chitose Kami et al., 2010; E. Liscum et al., 2014; Wahid, Ahmad, Nor, \& Rashid, 2017). Plants then integrate environmental information photomorphogenically to 
facilitate environmental adaptation critical to survival and conferring higher fitness (Casal \& Qüesta, 2018; Galen, Rabenold, \& Liscum, 2007; H. J. Lee, Park, Ha, Baldwin, \& Park, 2017; Serrano-Bueno et al., 2017).

Critical light cues are perceived via specialized light-absorbing proteins called photoreceptors (John M. Christie, Blackwood, Petersen, \& Sullivan, 2015; E. Liscum et al., 2014). Photoreceptors enable plants to detect not just the presence or absence of sunlight, but allow them to gauge specific light quality and quantity information: intensity, wavelength, direction, and duration (Fiorucci \& Fankhauser, 2017). There are at least five families of known photoreceptors in higher plants: phytochromes (phys), UV Resistance Locus 8 (UV8), cryptochromes (crys), phototropins (phots), and members of the zeitlupe family, each perceiving and responding to light in specific ways (Casal, 2013; Chaves et al., 2011; John M. Christie et al., 2015; Jenkins, 2014; Chitose Kami et al., 2010; E. Liscum et al., 2014; Petroutsos et al., 2016; Pudasaini \& Zoltowski, 2013; Zoltowski \& Imaizumi, 2014). With the exception of UV8, each family of photoreceptor is represented by multiple protein family members; for instance, there are five phytochromes (phyA through E) in Arabidopsis thalina (C. W. Whippo \& Hangarter, 2004). Additionally, there is crosstalk between many of the photoreceptor signaling pathways, which act synergistically and antagonistically and frequently alter back and forth depending on the environmental situation (Casal, 2000).

Plants use the information from their light environment to modify nearly every aspect of their growth from seed germination to reproduction to compete in a 
variable and competitive environment. Using information collected through photoreceptors, plants adapt their germination, pigment production, height, metabolism, leaf expansion, root architecture, flowering time, and numerous other responses (Casal, 2013; John M. Christie et al., 2015; R. M. R. M. Harper et al., 2001; Chitose Kami et al., 2010; Whitelam et al., 1998). Additionally, photoreceptors aid plants in regulating light-capture responses such as chloroplast re-localization, leaf positioning, and directional growth, which cannot be overstated given plants' dependence on photon capture as energy source (Fankhauser \& Christie, 2015; lino, 2006; S. I. Inoue et al., 2008; Kong \& Wada, 2016; E. Liscum et al., 2014; Stone et al., 2008). Altogether, the suite of photoreceptors equip sessile plants with a sophisticated method of perception that enables plants to modulate light capture and growth precisely throughout development, from germination to seed set.

Many of the aforementioned photomorphogenic responses have been described and studied for decades, some even by Charles Darwin (Kutschera \& Niklas, 2009). In recent years most studies have used the model plant Arabidopsis thaliana rather than investigating light responses in other plant taxa or agricultural species; largely due to the associated difficulties of unraveling the highly sophisticated signaling networks that are involved in light regulated developmental responses in crop species that lack the investigative tools associated with a model species. At the time this manuscript was written, a search of PubMed for "soybean light responses" yielded 110 papers devoted to 
the topic, and most are genome analyses while few look at physiological responses, whereas a search for "Arabidopsis light responses" yielded 1747. The domestication of plants has profound effects on the genetics and morphology of a given plant species, but the effect of domestication on photoreceptors or photoreceptor-mediated responses has been studied very little to date (Cockram et al., 2007). Meanwhile, the potential agricultural valuation of developing photoreceptors via synthetic biology or even classic breeding programs remains high due to the critical nature and wide scope of the developmental impact that photoreceptors have on plant growth and development (Celaya \& Liscum, 2007; Galen, Huddle, \& Liscum, 2004; Galen et al., 2007; R. M. R. M. Harper et al., 2001; lino, 2006; Mawphlang \& Kharshiing, 2017).

Glycine max, more commonly known as cultivated soybean, is one of the most important agricultural crops in the world, grown on an estimated $6 \%$ of crop-land worldwide with a global production of more than 230 million metric tons (Crane \& Boga, 2017; Hartman, West, \& Herman, 2011). Global G. max production has steadily increased since 1961 by nearly five percent annually and is expected to continue to increase with models predicting greater than 370 million tons by the year 2030 (Crane \& Boga, 2017). The constant growth in worldwide G. max production is a testament not only to its relative ease of cultivation, but also to the diverse products that can be extracted from its seeds.

Domestication had a profound effect on G. max diversity, which underwent a "domestication bottleneck" (Chacón-Sánchez \& Martínez-Castillo, 2017; Guo et 
al., 2010). The progenitor of G. max, Glycine soja, has an epicenter in eastern Asia, and may have far reaching potential to supplement the genetic diversity of G. max that was lost as a result of human domestication processes (Hyten et al., 2006; M. Y. Kim, Van, Kang, Kim, \& Lee, 2012; Y. H. Li et al., 2010; ShermanBroyles et al., 2014; Stupar, 2010). It is suggested by genetic analysis that $50 \%$ of genetic diversity and $81 \%$ of rare alleles were lost in the process of domestication of G. max, and approximately $60 \%$ of genes showed significant changes in allele frequency (Hyten et al., 2006). The morphological impact that domestication has had on the bushy and robust $G$. max is readily apparent; $G$. soja, in comparison, is a small viny plant that produces relatively small seeds encased in hard shells that are dispersed by seed pods that open when dried and must be scarified before germination.

The present study sought to characterize light responses associated with early seedling establishment in G. max and G. soja. Using G. soja in this study allowed us to determine if during the domestication process light responses were potentially altered or eliminated. It may be that variation was lost during the bottleneck event or through the pressures exerted by artificial selection. It is intriguing to speculate that valuable responses could have been reduced or eliminated during the domestication process and that reintroducing such light responses back into G. max could prove agriculturally beneficial. It was an initial goal of the research in this paper to discover any potential early photomorphogenic responses present in G. soja that are not present in G. max to provide a foundation for future scientific exploration. 


\section{Materials and methods}

\section{Seed stocks}

Glycine max was of the ecotype Williams 82. Glycine soja was of the ecotype PI407171.

\section{Growth conditions}

Both G. max and G. soja seeds were soaked in water for 20 minutes to imbibe before being sown in a vermiculite based soil (Sunshine mix). Ages of plants were measured from the start of imbibition to their harvest time. For G. soja seeds were scarified by chipping a small piece of the seed-coat away using the tip of a razor blade. The seeds were then placed in water for 20 minutes to imbibe and to ensure that the seed coats had been chipped sufficiently to allow water to penetrate the seed coat for germination to begin.

Light treatments were administered from above for both G. max and G. soja from a light source (see below) at a fluence rate of $50 \mu \mathrm{mol} \mathrm{m} \mathrm{m}^{-2} \mathrm{~s}^{-1}$ for 14 days from the time seeds were placed in soil until the seedlings were collected. Seedlings grown in darkness were placed in a wooden light-tight box in the same space where light treatments were performed so that temperature and humidity would be uniform across treatments.

\section{Light sources}

All blue and red light-treatments were administered with an in-house built LED bank. For each color 10 LEDs were placed in a grid and connected in series for a maximum output of approximately $50 \mu \mathrm{mol} \mathrm{m}^{-2} \mathrm{~s}^{-1}$ at $25 \mathrm{~cm}$ from the light source. 
All materials to construct the LED bank were purchased through LED Supply Co (Lakewood, CO). The specific LEDs used were: Red Cree XLamp Photo Red High Power Star (single configuration) and Blue Cree XLamp Royal Blue High Power Star (single configuration). The wavelength output of each LED was: blue $=450 \mathrm{~nm} \pm 10 \mathrm{~nm}$ and $\mathrm{red}=660 \mathrm{~nm} \pm 10 \mathrm{~nm}$.

Far-red light treatments were administered by filtering white light from an 80 -Watt Great Value ${ }^{\text {TM }}$ PAR38 Halogen Flood lightbulb through a $30 \mathrm{~cm}$ wide $\times 60 \mathrm{~cm}$ long $x 12 \mathrm{~cm}$ deep clear water bath to dissipate heat and a single sheet of far-red transmitting acrylic (Plexiglas ${ }^{\circledR}$ G 3143, www.eplastics.com). Resultant far-red light transmitted was $760 \mathrm{~nm} \pm 10 \mathrm{~nm}$.

White light was provided by 4 four-foot fluorescent General Electric Cool WhiteF34CWWM ECO EX Hg 34 Watt 4100K blubs.

The intensity of each light treatment was measured using a EPP2000 photometer purchased through StellarNet Inc. with the aid of Stellernet SpectraWiz software.

\section{Results}

Seeds were planted and grown for 14 days in darkness and in each of four light conditions: white, red, blue, and far-red. At the end of the 14 days of growth, seedlings were collected and multiple measurements recorded, including: root length, hypocotyl length, first internode length, and second internode length (Fig. 3.1). For certain light conditions, as will be discussed following, seedlings did not reach a given developmental stage (e.g., formation of second internode) during the 14-day growth period. The data collected facilitated a multitude of 
comparisons to be made between G. max and G. soja grown in darkness to seedlings grown in white, blue, red, or far-red light. 


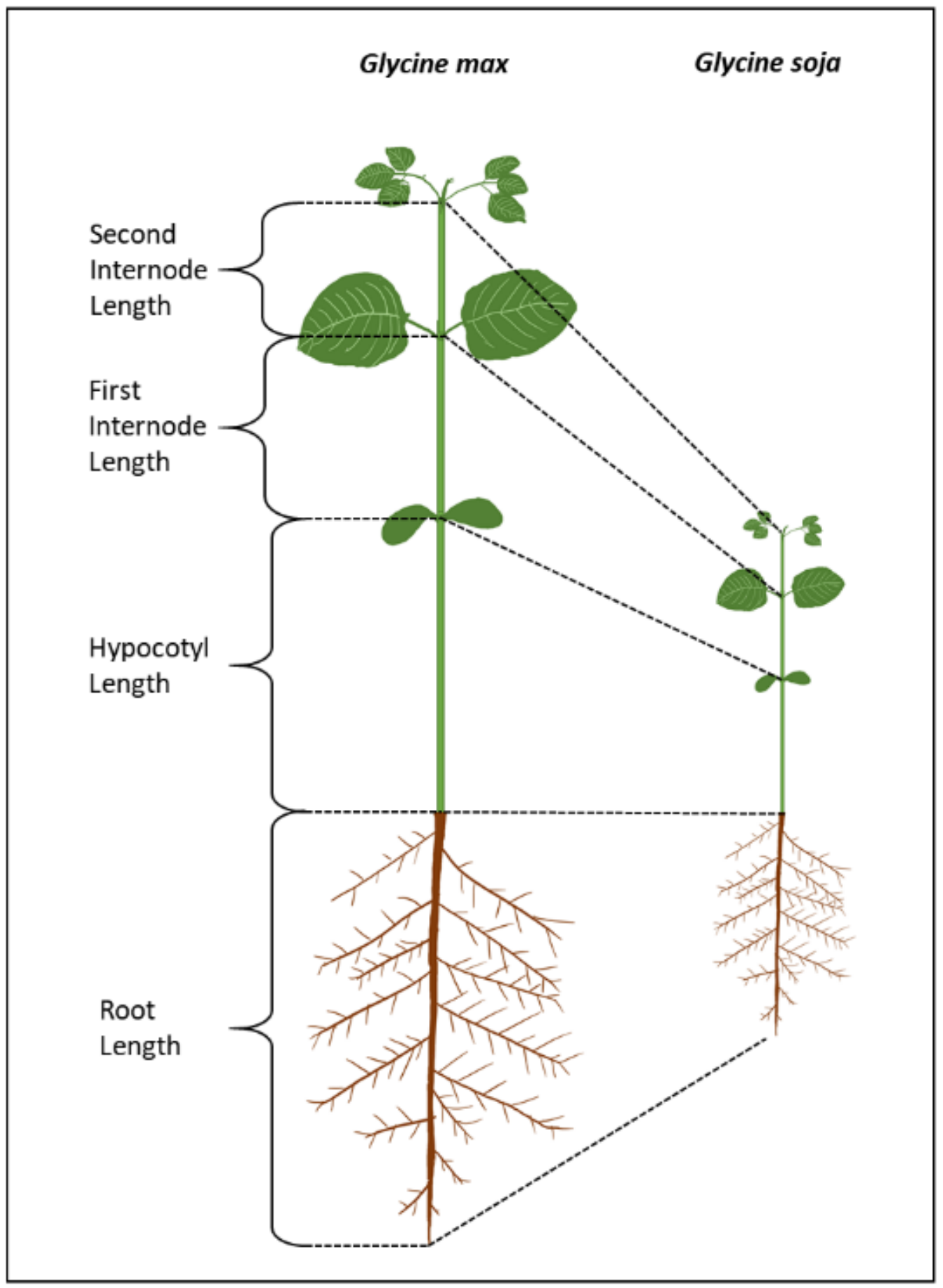

Figure 3.1 Drawing of two-week-old G. max and G. soja seedlings

After fourteen days growth in white, blue, red, or far-red light at a fluence rate of $50 \mu \mathrm{mol} \mathrm{m}^{-2} \mathrm{~s}^{-1}$, seedlings were collected and hypocotyl, first internode, second internode, and root length were measured 


\section{Responses of hypocotyls and first internode to white light}

Artificial white light, a representation of the full electromagnetic spectrum reaching the surface of the Earth from the sun, represents the typical light environment encountered by growing plants. It would be an oversimplification to state that all plants growing in white light experience the same light environment. Seasons, canopy shading, and object proximity are examples of conditions that alter the light quality a plant encounters, and as described above, plants have adaptive mechanisms of detecting and responding to the environmental conditions available to them. However, growing seedlings in white light provides a photomorphogenic standard to serve as an experimental backdrop to set comparisons against in monochromatic light conditions.

In $50 \mu \mathrm{mol} \mathrm{m} \mathrm{m}^{-2} \mathrm{~s}^{-1}$ white light, G. max and G. soja both exhibit nearly $50 \%$ hypocotyl inhibition (Fig. 3.2. A and B). The first internode exhibits nearly $120 \%$ growth stimulation in G. $\max$, whereas G. soja only has $25 \%$ growth simulation (Fig. 3.2 A and B). The total stem length in G. max was slightly reduced in white light, while slightly stimulated in G. soja (Fig. 3.2A and B).

A comparison of the average lengths of seedling regions (Fig. 3.2. C) reveals some additional differences between G. max and G. soja. For example, total seedling length of G. max in white light is contributed to by hypocotyls, first internode, and second internode, whereas in G. soja most of the total stem length is contributed by the hypocotyl and first internode, with very reduced length second internodes. When comparing the proportion of seedlings 
producing a first internode in white light and darkness, G. max produced a first internode at nearly equal percentages in white light and in darkness, whereas $G$. soja rarely produced a first internode in darkness but produced a first internode in white light in $75 \%$ of seedlings (Fig. 3.2. D). 
A)

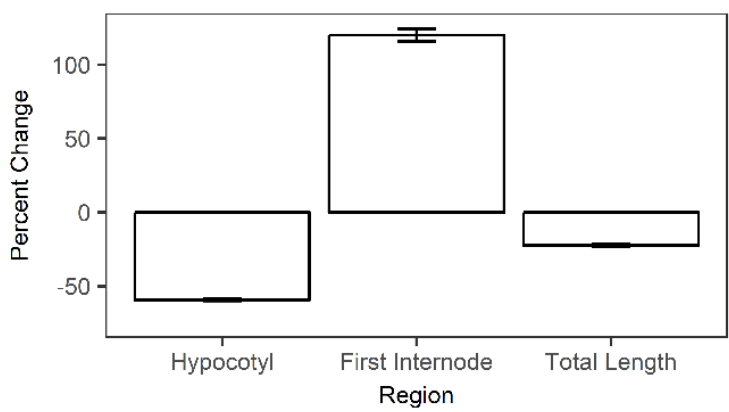

C)

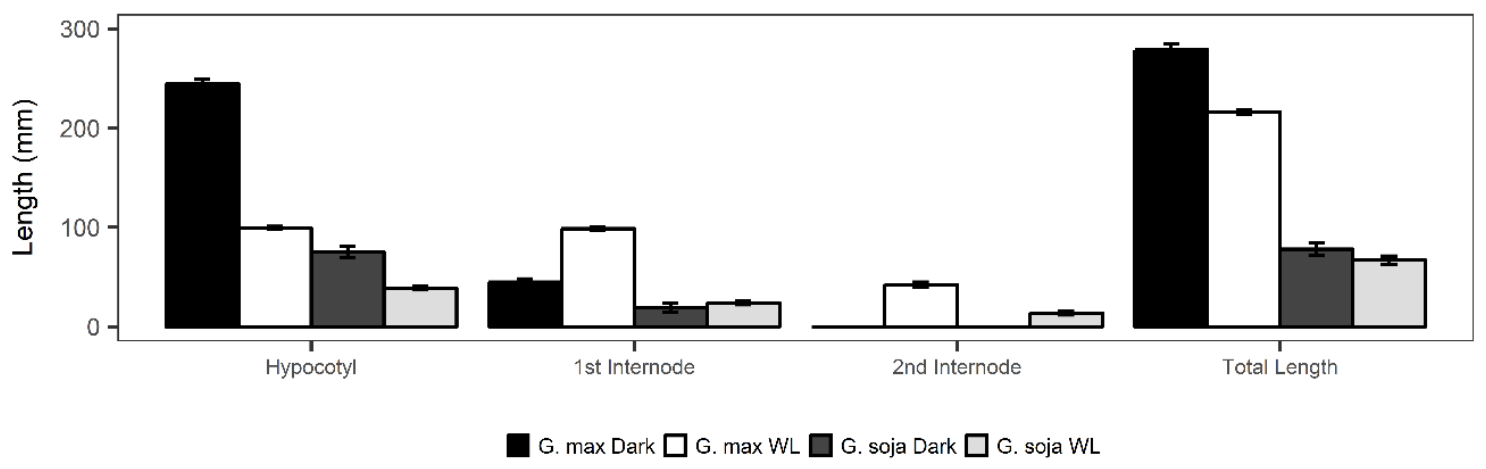

D)

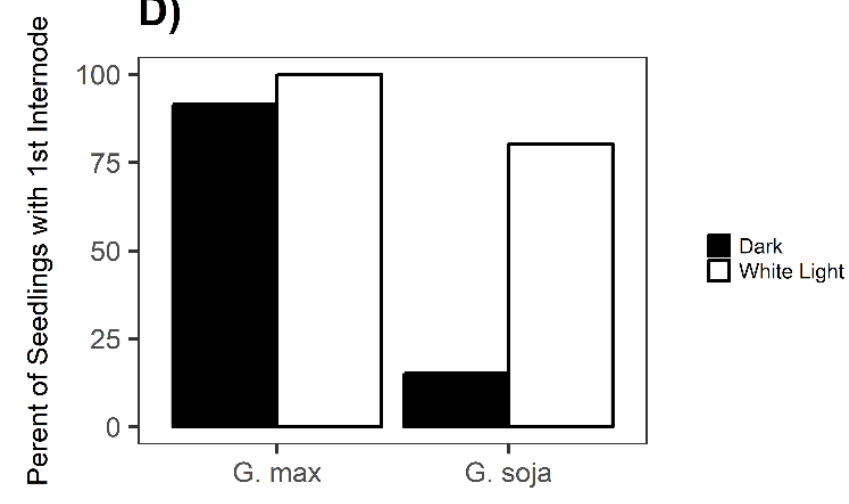

B)

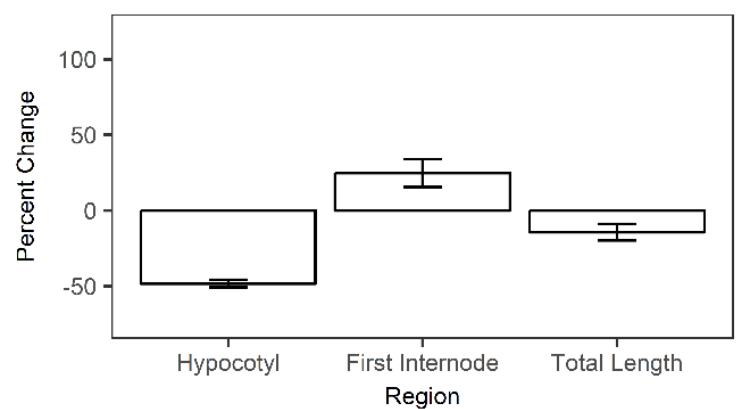




\section{Responses of hypocotyls and first internode to blue light}

Photomorphogenesis associated with blue-light treatments has been wellcharacterized in $A$. thaliana while relatively little research has been performed in soybean. In $A$. thalania seedlings grown in $50 \mu \mathrm{mol} \mathrm{m}{ }^{-2} \mathrm{~s}^{-1}$, blue light treatments exhibit greater than $80 \%$ hypocotyl growth inhibition relative to dark-grown seedlings (Lin et al., 1998). In contrast to $A$. thaliana, neither Glycine species exhibit that level of growth inhibition in blue light. Interestingly, when total stem length (hypocotyl, first, and second internode lengths) for both G. max and $G$. soja was examined in blue light, neither exhibited a dramatic difference compared to dark-grown seedlings. The hypocotyl region showed approximately $44 \%$ reduction in length relative to dark-grown hypocotyls for $G$. max and an approximately $32 \%$ reduction in G. soja (Figure $3.3 \mathrm{~A}$ and B). The reason for these phenotypic similarities becomes obvious when one looks at the elongation of first and second internode. The length of the first internode exhibited a marked growth stimulation in blue light compared to dark-grown seedlings, with G. max exhibiting an approximately $82 \%$ growth stimulation relative to dark and G. soja exhibiting an approximately $122 \%$ growth stimulation (Fig. 3.3. A-C). This data taken together indicates that for both G. max and G. soja, blue light-induced morphogenesis leads to growth suppression below the cotyledons and growth stimulation above it. 
A)

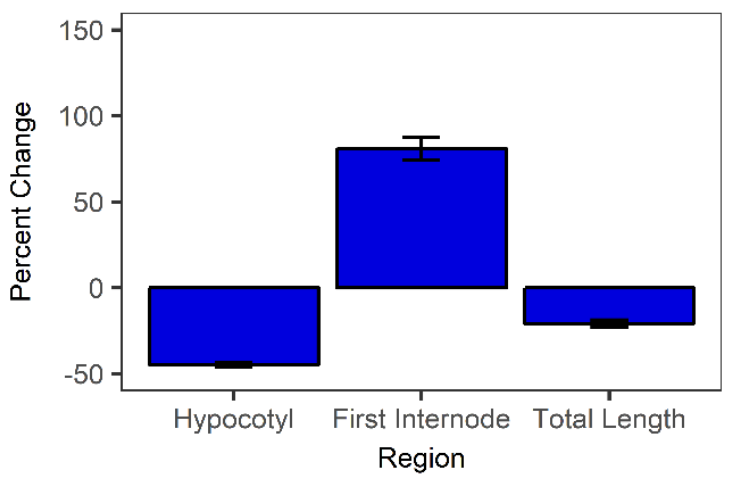

C)

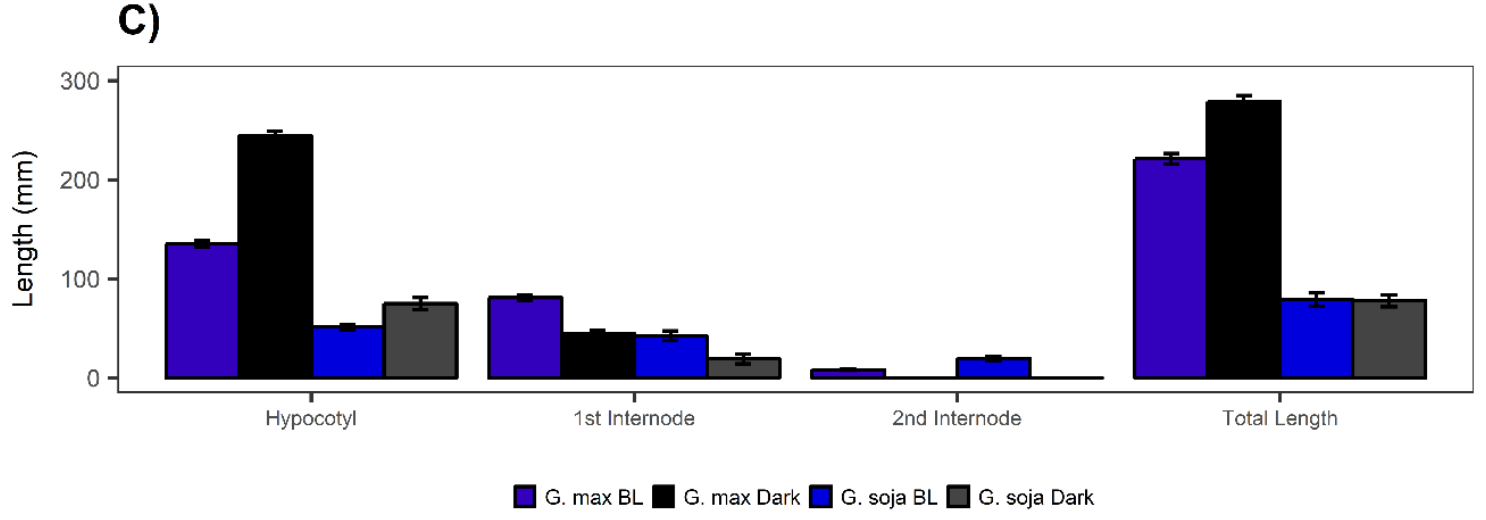

B)

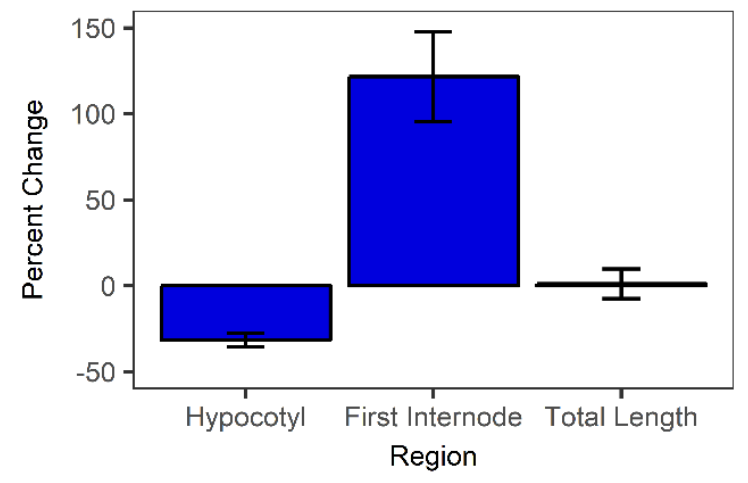

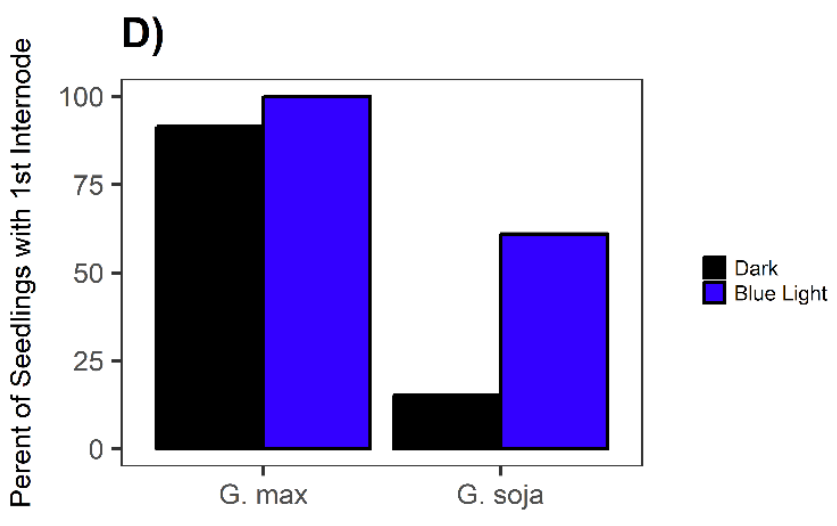

E)

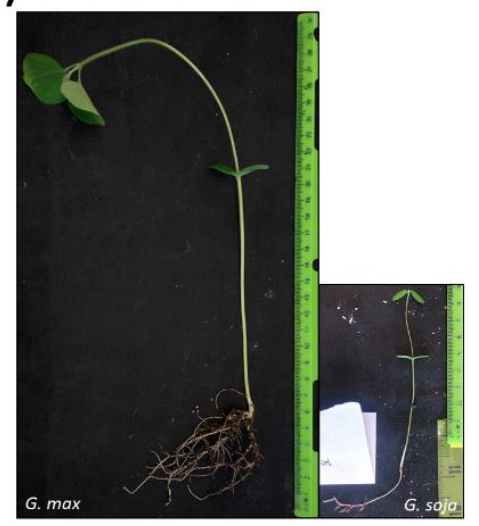

Figure 3.3 Blue light growth is inhibited in the hypocotyl but induced in the second internode

Relative growth inhibition or induction in G. $\max (A)$ and G. soja (B) in $50 \mu \mathrm{mol} \mathrm{m}^{-2} \mathrm{~s}^{-1}$ blue light after 14 days and. For both G. max and G. soja, hypocotyls showed a reduction in growth compared to seedlings grown in darkness. Measurements of average length in blue light (C) indicates most of the total length of seedlings was contributed by the hypocotyl and $1^{\text {st }}$ internode regions. The percentage of seedlings developing a first internode (D). Representative images of G. max and G. soja. Error bars represent \pm SE for a minimum 90 seedlings from a minimum of 3 replicate experiments $(E)$. 


\section{Response of hypocotyl and first internode to red light}

Like blue light, red light is a key energy source for photosynthesis, and therefore it is important for plants to be able to perceive red light and adapt their growth to environments with varying levels of red light. Red and far-red light are detected via the phytochrome photoreceptors (Whitelam et al., 1998). In A. thaliana the phytochrome family is composed of five members, phyA-E (Clack et al., 1994). The phytochromes, upon light perception, undergo a photoreversible change that initiates signaling (M. Chen \& Chory, 2011; Franklin et al., 2005; Ulijasz et al., 2010). Phytochromes can perceive both red and far-red light, functioning as a binary molecular switch (M. Chen \& Chory, 2011). Physiologically, phytochromes regulate a myriad of critical responses, including germination, greening, cotyledon opening, hypocotyl growth inhibition, de-etiolation, shade avoidance, day length detection, and flowering time (M. Chen \& Chory, 2011; Franklin \& Whitelam, 2005).

Neither G. max or G. soja displayed hypocotyl growth that would be considered abnormal under continuous red light when compared to the growth of $A$. thaliana (Hennig, Poppe, Unger, \& Schäfer, 1999). A. thaliana displays around 40\% hypocotyl growth inhibition grown in similar red light conditions as used in the present study (Hennig et al., 1999; Koornneef, Rolff, \& Spruit, 1980; Whitelam et al., 1998). G. max had approximately $45 \%$ hypocotyl growth reduction in red-light grown seedlings compared to dark-grown seedlings (Fig. 3.4. A) and G. soja approximately $28 \%$ (Fig. 3.4. B). In the first internode G. max and G. soja showed approximately a $190 \%$ and $123 \%$ growth stimulation (Fig. 3.4. A) 
respectively compared to dark-grown seedlings (Fig. 3.4. A and B). However, factoring in all regions of the stem, the total length of the stem was not significantly stimulated or inhibited in G. max and only slightly stimulated in G. soja relative to dark-grown seedlings (Fig. 3.4. A and B). Looking at the data so far discussed, we see a similar pattern to that observed for blue light-induced developmental responses where hypocotyl growth is inhibited but growth is stimulated in more aerial portions of the seedlings. 
A)

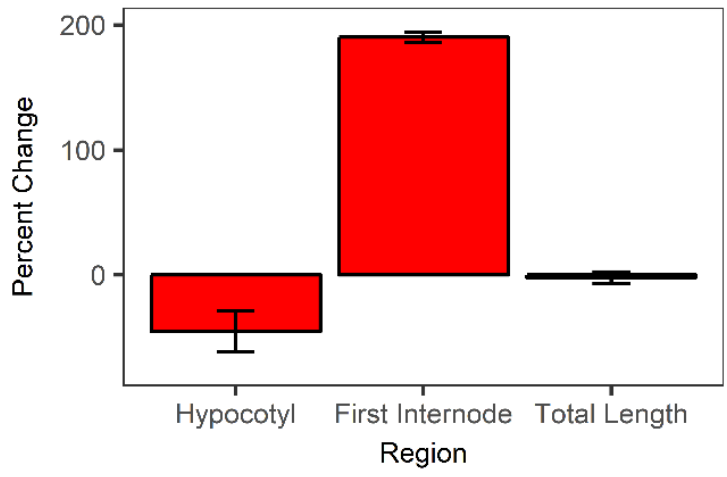

B)

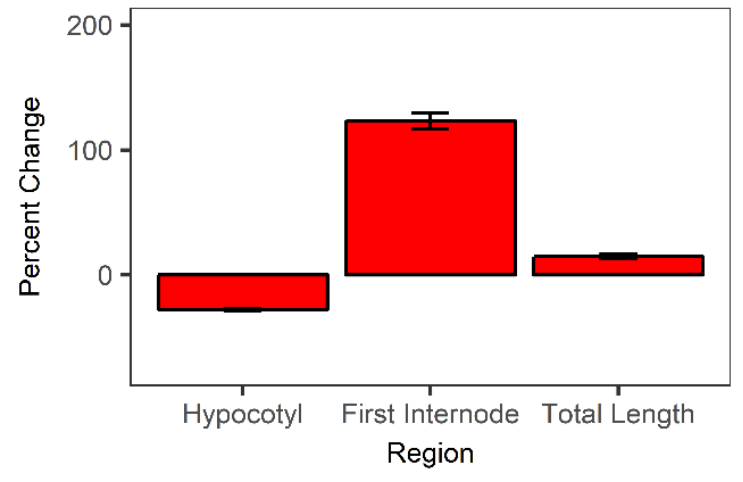

C)

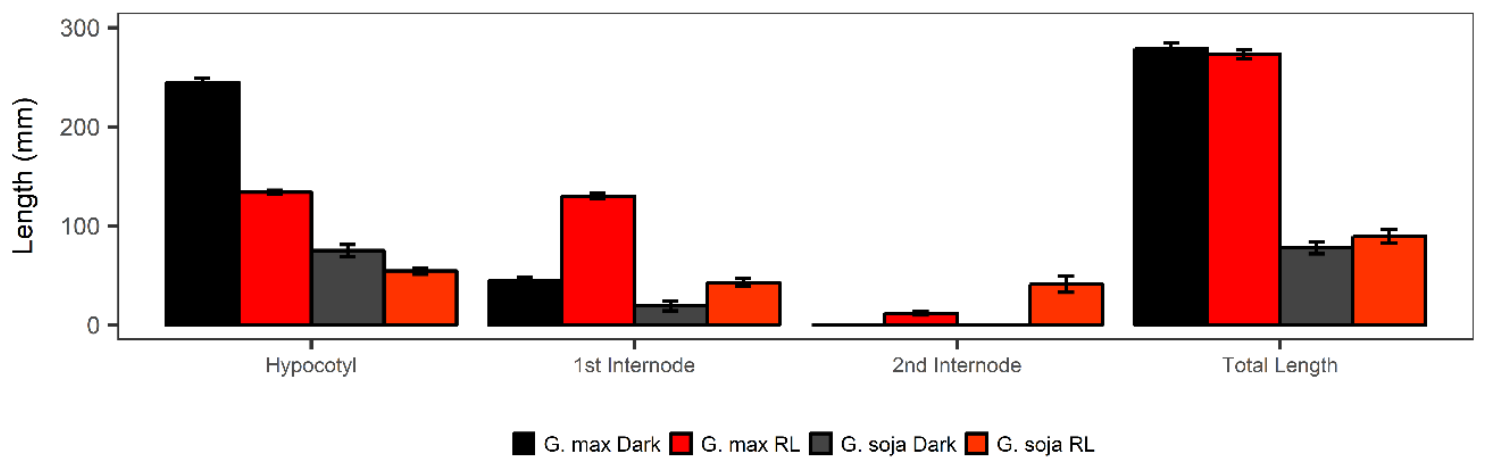

D)

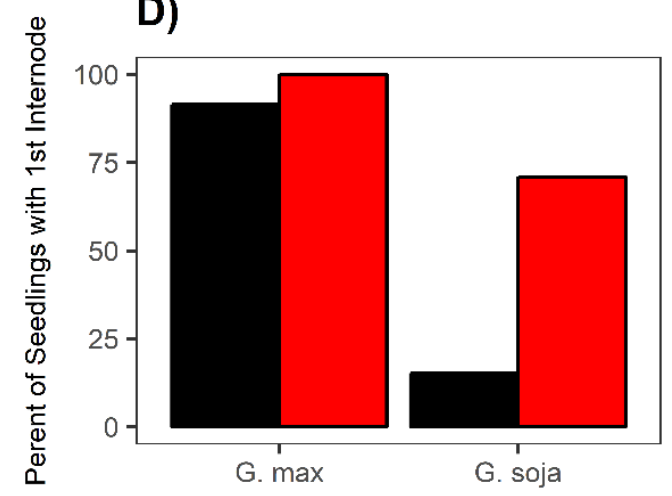

E)

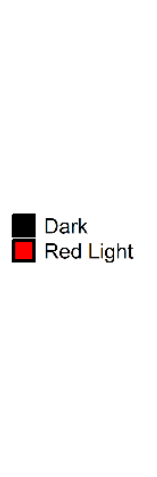

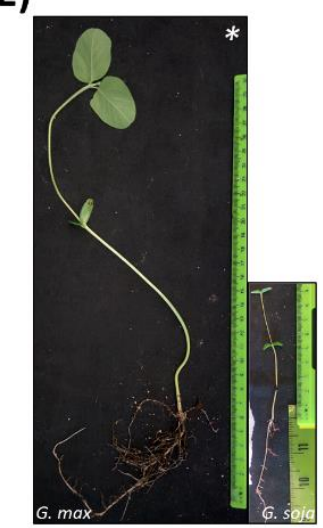

Figure 3.4 Red light growth is inhibited in the hypocotyl but induced in the first internode

Relative growth compared to dark control in G. $\max (A)$ and G. soja (B) in $50 \mu \mathrm{mol} \mathrm{m}^{-2} \mathrm{~s}^{-1}$ red light after 14 days indicates that both $G$. max and G. soja, hypocotyls exhibit inhibition whereas the first internode is stimulated. Measurements of average length in red light (C) indicates most of the total length of seedlings was contributed by the hypocotyl and $1^{\text {st }}$ internode regions. The percentage of seedlings developing a second internode (D) shows that G. max is more likely to produce both a $1^{\text {st }}$ internode compared to G. soja, but the difference is not as large as for seedlings grown in blue light. Representative images of G. max and G. soja $(E)$. Error bars represent \pm SE for a minimum 90 seedlings from a minimum of 3 replicate experiments. *indicates seedling was grown in blue light rather than red light. 


\section{Responses of hypocotyl and first internode to far-red light}

Exposure to continuous far-red light inhibits growth in the hypocotyls of $A$. thaliana seedlings, resulting in seedlings displaying a nearly complete growth inhibition that is more dramatic than the light-induced hypocotyl growth inhibition observed in either blue light or red light (Whitelam et al., 1998). The far-red lightinduced hypocotyl growth inhibition is mediated by the phyA photoreceptor, which is demonstrated by mutants lacking phyA developing along a dark developmental plan with elongated hypocotyls (Whitelam et al., 1998). Additionally, far-red light treatments stimulate phyA mediated cotyledon opening (Whitelam et al., 1998). This study indicates that G. max and G. soja develop along a distinct photomorphogenic programs in far-red light (Fig. 3.5. A and B) compared to far-red development observed in A. thaliana. Our data demonstrated that both G. $\max$ (Fig. 3.5. A) and G. soja (Fig. 3.5. B) had a total stem length not very different from dark-grown seedlings.

Further comparison of photomorphogenic development between G. max and G. soja reveals that despite the total length of the seedlings not being substantially different from each other, G. max and G. soja are operating along largely distinct developmental programs when their growth is analyzed on a per region rather than total length basis. When hypocotyl lengths are examined, we observe moderate inhibition in G. $\max (47 \%)$, while only a limited inhibition in G. soja (16\%) relative to plants grown in darkness (Fig. 3.5. A and B). Interestingly, the first internode in G. max exhibits dramatic growth stimulation in far-red light seedlings relative to dark-grown seedlings (129\%), whereas G. soja showed 
almost no difference in internode growth between dark- and far-red-grown seedlings (Fig.3.5 A and B). Overall, while G. max exhibits a fair amount of elongation regulation by far-red light, G. soja appears largely unresponsive (Fig. $3.5 \mathrm{C})$.

The differences in far-red light responsiveness between Glycine species examined here is further illustrated when one examines the development of the first internode more closely. The percentage of seedlings grown in far-red light developing a first internode in G. soja was $59 \%$ compared to $100 \%$ in G. max (Fig. 3.5 D). Dark-grown G. soja seedlings developed a first internode in only $15 \%$ of seedlings, whereas dark-grown G. max seedlings developed a first internode in $91 \%$ of seedlings (Fig. $3.5 \mathrm{D}$ ). The relative growth inhibition/stimulation, average lengths, and percentage of seedlings at a particular developmental stage implies that far-red light perception initiates different light programs in G. max and G. soja that ultimately result in average lengths only slightly reduced compared to dark grown counterparts. 
A)

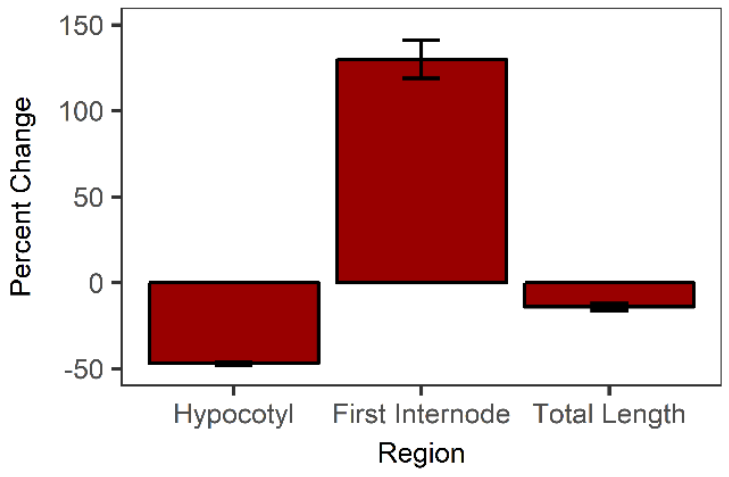

B)

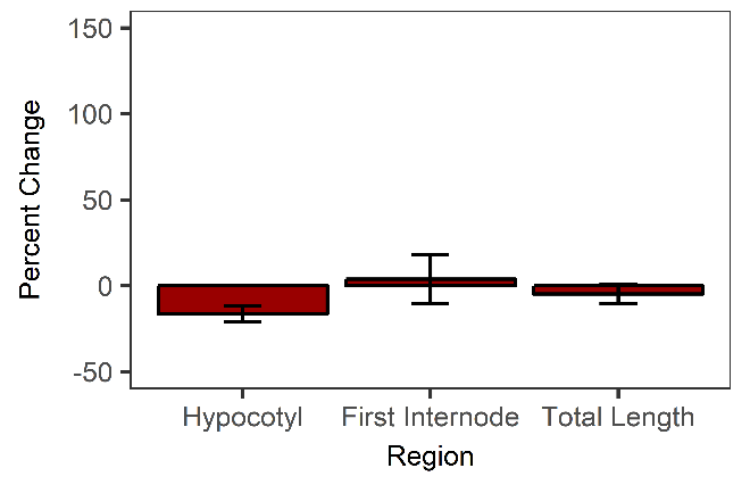

\section{C)}
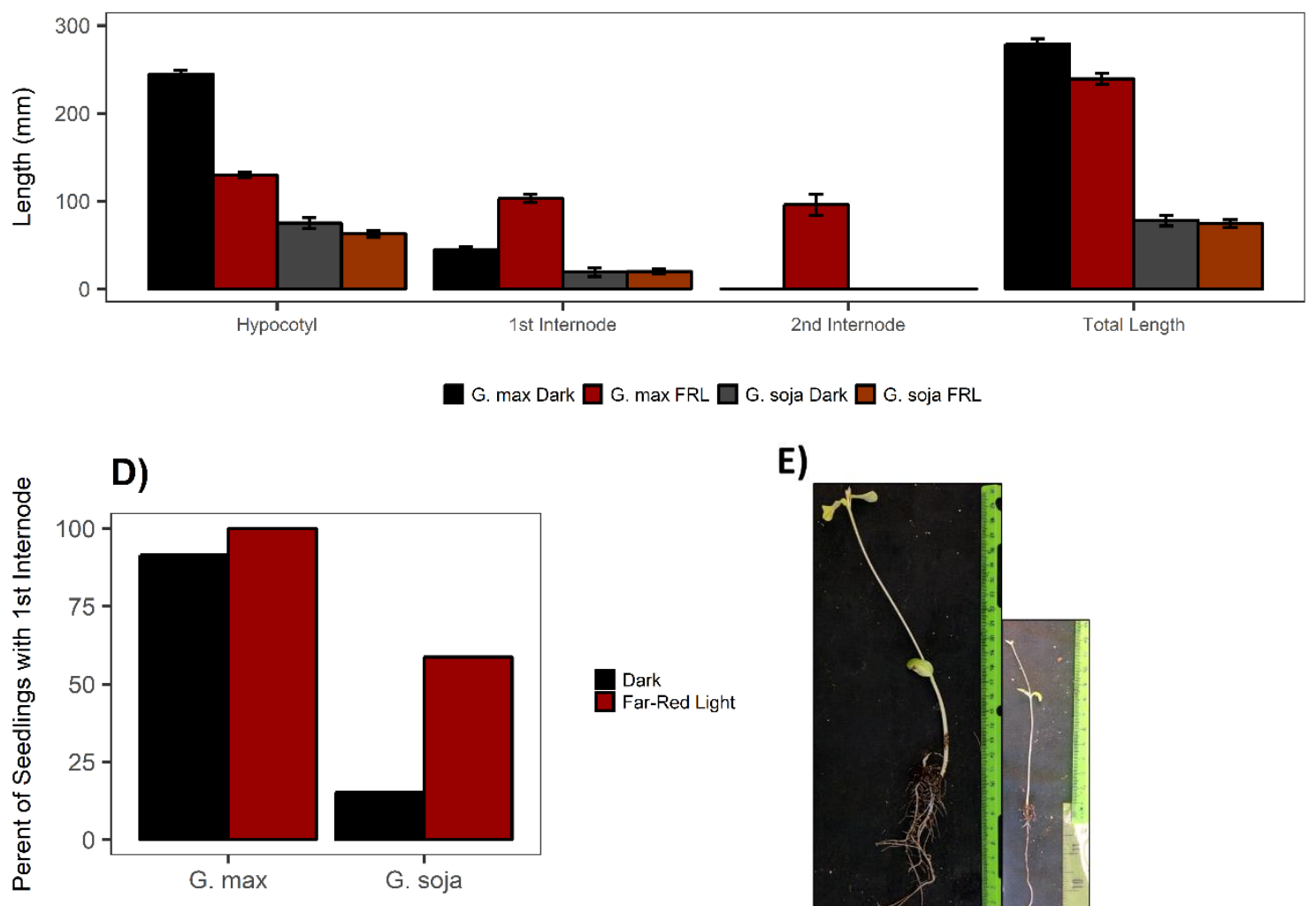

E)

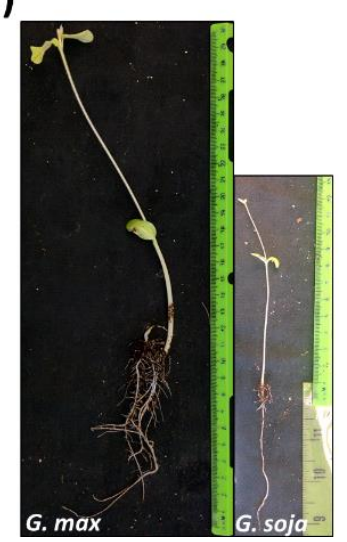

Figure 3.5 Far- red light growth is inhibited in the hypocotyl but induced in the second internode for G. max but not for G. soja

Relative growth compared to dark controls in G. $\max (A)$ and G. soja (B) in $50 \mu \mathrm{mol} \mathrm{m} \mathrm{m}^{-2} \mathrm{~s}^{-1}$ far-red light after 14 days. Measurements of average region lengths in far-red light (C) indicates a majority of total seedling length is contributed by the hypocotyl and first internode regions. The percentage of seedlings developing a first internode (D) demonstrates that G. max is more likely to produce both a $1^{\text {st }}$ internode and that $G$. soja. Representative images of $G$. max and $G$. soja (E). Error bars represent $\pm S E$ for a minimum 90 seedlings from a minimum of 3 replicate experiments. 


\section{Development of second internode under different light qualities}

Not surprisingly, not all seedlings reached the same stages of development. In the absence of light, seedlings grow according to a skotomorphogenic program, investing heavily in elongation of the hypocotyl while not investing in growth above the cotyledons, pigment production, or leaf development. None of the seedlings in either G. max or G. soja produced a second internode in darkness (data not shown). The absence of a second internode prohibits calculating relative growth inhibition for the second internode. However, comparisons of second internode length among light treatment conditions provide insight into light-regulated second internode development.

In white light G. max produced a second internode in $95 \%$ of seedlings while $G$. soja produced a second internode in $62 \%$ of seedlings (Fig.3.6).

In comparison to white light, G. max produced a second internode to varying degrees in monochromatic light conditions: in blue light (57\%), red light (94\%), and far-red light (51\%) (Fig. 3.6). G. soja exhibited more restricted development of the second internode compared to G. max in all light conditions, producing only few second internodes in blue light (8\%), red light (24\%), and far-red light (0\%) (Fig. 3.6). The general pattern of second internode development is consistent for G. max and G. soja; red light promoted the largest second internode development, while far-red light produced the least and blue light was somewhat intermediate. 


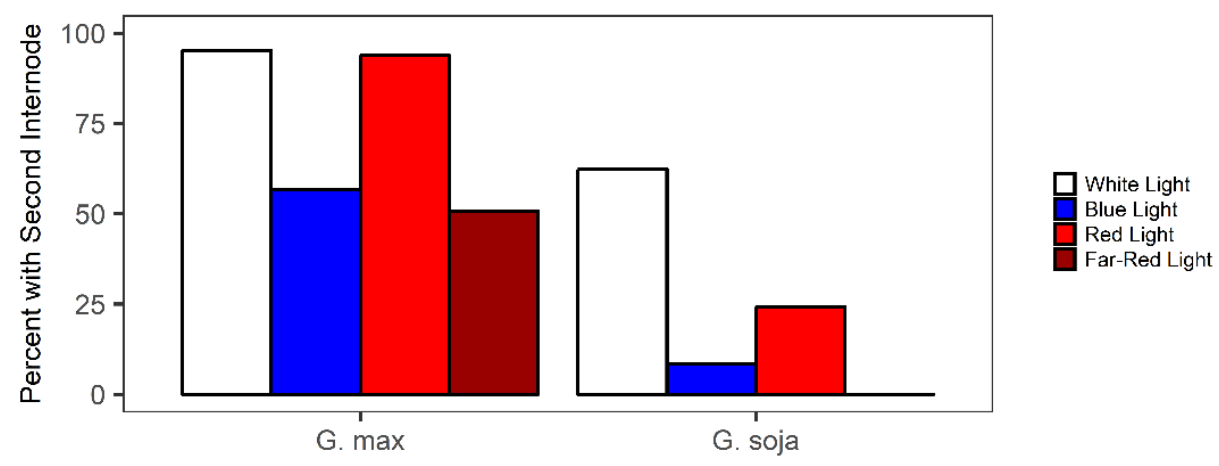

Figure 3.6 Percent of seedlings producing a second internode in various light qualities

Second internode length in G. max and G. soja in $50 \mu \mathrm{mol} \mathrm{m}^{-2} \mathrm{~s}^{-1}$ white, blue, red, and far-red light. Data are from a minimum of 90 seedlings from at least 3 replicate experiments. 


\section{Root elongation in response to different light conditions}

At first it seems counterintuitive that light perception would alter the growth of roots, but in seedling establishment the growth that is occurring beneath the ground is as important as growth above the ground. Seedlings germinating in the soil must appropriately invest their seed reserve during establishment of an autotropic growth habit. To establish an autotropic growth pattern, aerial tissues must break through the soil surface, initiate chlorophyll production, develop leaves, and commence photosynthesis (Eckardt, 2001; Josse \& Halliday, 2008).

To achieve vital autotropic capacity, resources must be allocated to aerial rather than subterranean tissues. This is reflected in the typical pattern of growth characteristic of skotomorphogenic seedlings where stem tissue elongates and there is minimal root elongation or development. In contrast during the photomorphogenic shift to autotrophic growth seedling stem growth is inhibited with an accompanying progression of leaf development, and roots elongate and branch (Eckardt, 2001; Josse \& Halliday, 2008).

The study shows that some of the most dramatic differences between dark and light-grown soybean seedlings occur in the roots. In all light conditions examined, G. soja had increased root length relative to dark-grown seedlings. In white light G. soja roots were $109 \%$ longer than in darkness; in blue light roots were $57 \%$ longer than in darkness; in red light roots were $61 \%$ longer than in darkness; and in far-red light roots were 37\% longer than in darkness (Fig. 3.7 A). G. max lightinduced root elongation was much less dramatic than that observed in G. soja. In comparison, to dark-grown G. max seedlings, root elongation in white light was 
$28 \%$ longer; in blue light roots were $21 \%$ longer; in red light roots were not much longer; and in far-red light roots were only $8 \%$ longer (Fig. 3.7A).

It is interesting to note that roots are not necessarily shorter in G. max than in G. soja but are more likely stimulated in dark-grown seedlings (Fig. 3.7 B). When comparing G. max and G. soja roots from an average length basis, we see that the average lengths of roots in $G$. max is only somewhat shorter in darkness as compared to light-grown seedlings, indicating that G. max seedlings are producing roots in darkness. G. soja, in comparison, produces roots once light has been perceived. This trend is consistent for the growth patterns observed in aerial portions of the seedlings, where G. max produced a second internode in darkness. These data again suggest that $G$. max is growing in a somewhat constitutively photomorphogenic state relative to G. soja, which is generally more responsive to light stimulation. 
A)

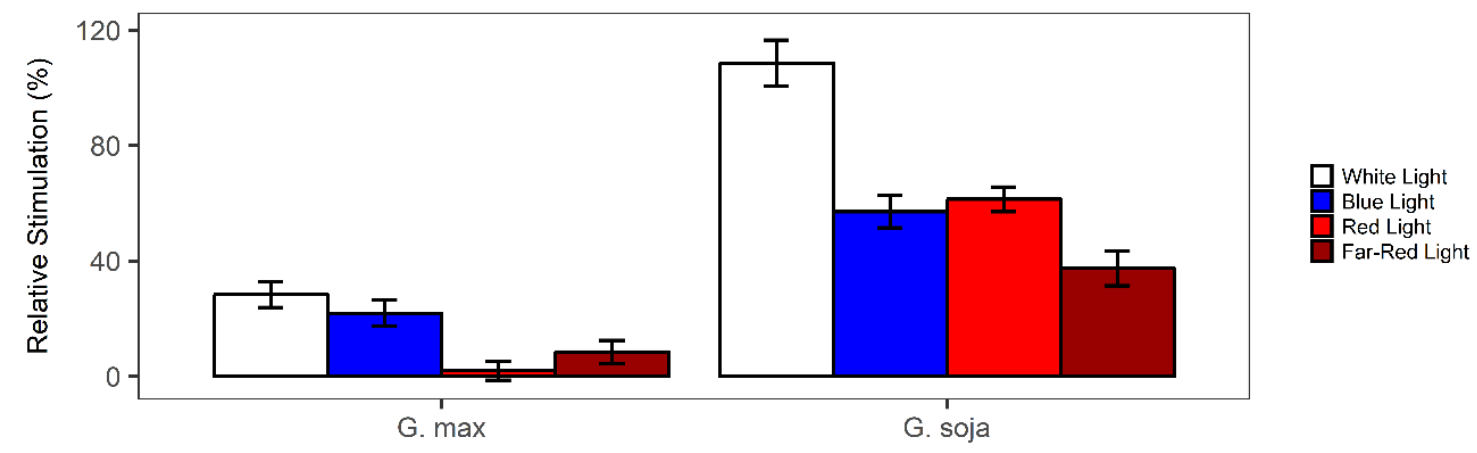

B)

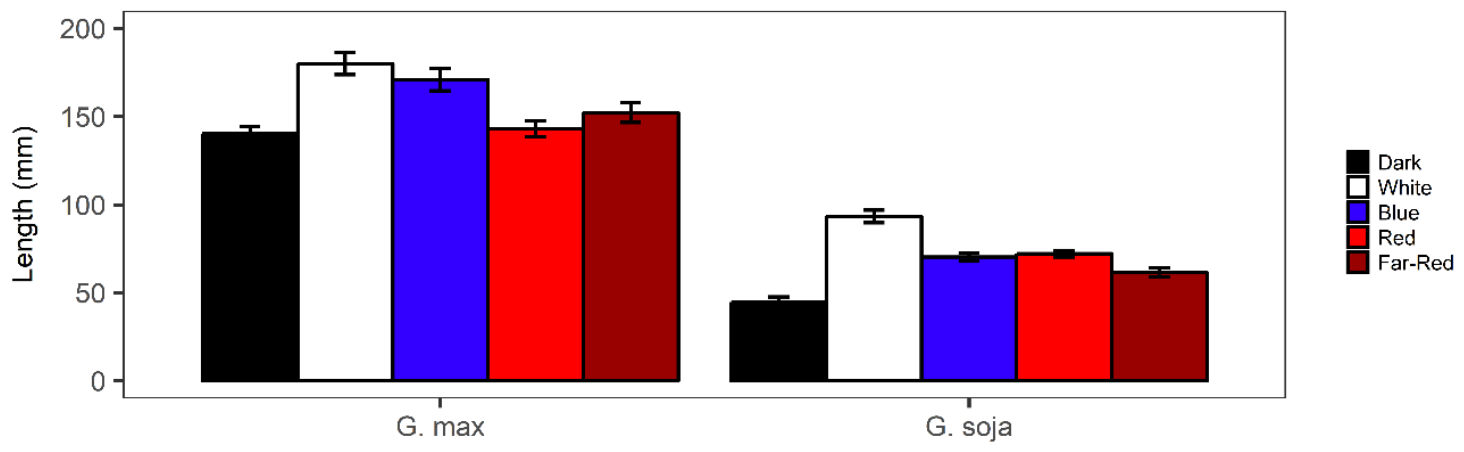

Figure 3.7Root length in G. max is stimulated in white, blue, and far-red light but not stimulated in red light, whereas G. soja is stimulated in all light conditions and more responsive to light that G. $\max$

Light induction of root elongation by blue, red, and far-red light in G. max and G. soja (A). Average length of roots grown under all light conditions (B). Error bars represent \pm SE for a minimum 90 seedlings from a minimum of 3 replicate experiments. 


\section{Discussion}

\section{Growth in white light}

White light is the natural light condition seedlings grow into as they emerge from soil into the air, but plants facilitate growth modulation by sensing and responding to variations in blue, red, and far-red wavelengths within white light. The photomorphogenic development of G. max and G. soja in white light illustrates the overall integrated photomorphogenic program that each of the two Glycine species follow. Domesticated G. max responds more intensely to white light than its ancestral progenitor G. soja, exhibiting stronger hypocotyl inhibition and stronger internodal stimulation (Fig. 3.2. A, B, and D). This data suggests that domestication has generally enhanced protomorphic responses to white light and that light regulated development has been selected for, not against, during domestication. This finding is intriguing given the domestication bottleneck that soybean went through and provides a striking example of the power of agricultural breeding to alter crop species (Guo et al., 2010; Hyten et al., 2006)

\section{Growth in blue light}

Development of G. max and G. soja under blue light indicates that that blue light is perceived as a signal to invest the early, limited seedling resources into aerial organ development while reducing energy to hypocotyl growth (Fig. 3.3. A and B). This finding is somewhat intuitive; once blue light-energy is perceived, resources are no longer limited, and investing in aerial development ensures maximal light capture. However, comparison of $G$. max with pre-domesticated $G$. soja illustrates that blue light-regulation is different between these species; $G$. 
max invests more into development of first and second internodes compared to G. soja (Fig. 3.3. D, Fig. 3.6), suggesting that domestication has resulted in $G$. max seedlings that are quick to expend energy to develop aerial organs as compared to G. soja.

\section{Growth in red light}

In red light both G. max and G. soja exhibit minor inhibition in hypocotyls but drastic stimulation in the first internode (Fig.3.4 A and B), suggesting that red light perception inhibits growth in lower regions while stimulating growth in the aerial-most regions. However, several notable differences present themselves that indicates domestication has altered red light development responses. As shown in Fig. 3.4 A, the first internode in G. max is nearly twice as stimulated relative to seedlings grown in darkness as compared to $G$. soja. The percentage of seedlings developing a first internode in darkness and in red light is similar in G. max suggesting that $G$. max develops a first internode before sensing red light and upon red light perception induces dramatic elongation in the first internode (Fig. 3.4. A and D). This implies that domestication has resulted in seedling development anticipating light rather than commencing development as a response to light. G. soja in contrast seldom develops a first internode in darkness, but produces an elongated internode in red light (Fig. 2.4. D). These developmental responses make adaptive sense for G. max in the field where light availability is certain and risk of starvation minimal. 


\section{Growth in far-red light}

G. max and G. soja respond vastly different to continuous far-red light and represents one of the greatest differences between Glycine species. G. max develops in response to far-red light, with short hypocotyls (Fig.3.5 A), whereas G. soja is nearly insensitive to far-red light with seedlings not differing substantially in hypocotyl length, first internode length, total length, or formation

of a second internode (Fig. 3.5. B and C, Fig. 3.6). It is intriguing that G. max and G. soja vary dramatically in far-red light responses and that G. max is responsive while $G$. soja is relatively unresponsive, conflicting with the prediction that domestication results in loss of light responsiveness.

\section{Growth of roots discussion}

The data presented in (Fig.3.7) suggest that in all light conditions, root growth is stimulated compared to roots in dark-grown seedings in G. soja, but in G. max light is far less efficient in inducing root elongation. In red and far-red there is almost no root growth stimulation in G. $\max$, whereas $G$. soja exhibited approximately $60 \%$ and $40 \%$ root-growth simulation, respectively (Fig.3.7). This finding may suggest a modification to the phytochrome photoreceptor signaling pathway during the domestication process. It is also interesting to note that $G$. max root-growth was never highly stimulated in any light condition suggesting that the processes of artificial selection reduced the light-induced root-growth response observed in G. soja. Perhaps uniform planting of seedlings and the tilling of soil decreases the necessity for light-induced root stimulation, which has been lost during the domestication of of G. max, possibly through gene or gene 
function loss during domestication. Future studies may someday elucidate what causes the striking differences in light induced root elongation observed between G. max and G. soja.

\section{Conclusion}

This study describes the early responses of $G$. max and G. soja seedlings to white light and monochromatic light treatments, identifying multiple surprising differences in photomorphogenesis between a domesticated crop species and its progenitor species. Future studies could use the phenotypes described in this manuscript in genetic screens of mutant populations in G. max and/or G. soja in studies seeking to identify light-regulated responses in developing seedlings. Due to the high impact nature of morphogenesis in seedling establishment, we propose additional investigation into the genetic control of light responses in Glycine species.

\section{Acknowledgements:}

Thanks is due to Dr. Bing Stacey for providing Williams 82 G. max seeds and for very valuable advice and to $\mathrm{Dr}$. Andrew Scaboo for G. soja seeds and the knowledge to grow and plant them. Additional thanks to Mannie Liscum for critical insights and edits throughout the drafting of this manuscript and in data preparation. 


\section{Chapter 4 CONCLUSIONS}

Light is of critical importance to plants for development. Plants use the information in light to perceive their environment and can respond by adapting their growth appropriately. Plants facilitate critical light perception via several classes of photoreceptors that work individually and cooperatively to mediate photomorphogenic development. Light signaling responses are critical not only in non-cultivated plants that compete in environments consisting of diverse species but also in cultivated crop species with nearly homogenous growing environment. While research has served to advance our understanding of the mechanisms of light-induced responses from perception to response, much is still unknown. In the crop species G. max, known genetic bottleneck has occurred during the domestication process with large losses in genetic diversity occurring. While modern breeding practices have provided impressive advances in G. max yield increase, there is likely greater potential to be unleashed in the genetic material of $G$. soja. To date, little research has investigated the effects of light signaling in early seedling development in the important crop species G. max.

Another area within the field of plant light perception that has not yet been elucidated is the modulation of blue light phototropism by the red light photoreceptor phyA. For decades, phyA has been known to function to modulate phototropic curvature and other phot mediated responses yet direct routes for this modulation have not been revealed mechanistically. Uncovering the ways that phyA and phot1 signaling is intertwined would provide an important piece of 
the signaling for several of the most important directors of plant growth and development.

\section{phyA $^{\mathrm{K} 555 \mathrm{R}, \mathrm{K} 603 \mathrm{R}}$ lines are degraded in red light and exhibit far-red}

\section{light-induced hypocotyl growth inhibition}

phy $\mathrm{A}^{\mathrm{K} 555 \mathrm{R}, \mathrm{K} 603 \mathrm{R}}$-transgenic lines exhibit normal far-red light induced hypocotyl growth inhibition and are degraded in red light (Fig. 2.1). Mutation of phyA in the two lysine residues $\mathrm{K} 555$ and $\mathrm{K} 603$ to arginine does not alter the far-red induced, phyA mediated growth inhibition response or red light-induced degradation of phyA. These results are not entirely unexpected because the lysine residues targeted for ubiquitination were shown to be blue light-dependent, not red or far red dependent. These findings also indicate that phy $\mathrm{A}^{\mathrm{K} 555 \mathrm{R}, \mathrm{K} 603 \mathrm{R}}$ lines are functional in several classic phyA responses.

\section{phy $^{\mathrm{K} 555 \mathrm{R}, \mathrm{K} 603 \mathrm{R}}$ lines display normal blue light-induced}

\section{phototropism under low and very low intensity conditions}

Very low and low intensity directional blue light induces phototropic curvature but is reduced in the absence of phyA. Phototropic assays in very low and low intensity blue light were performed with phy $\mathrm{A}^{\mathrm{K} 555 \mathrm{R}, \mathrm{K} 603 \mathrm{R}}$ lines and revealed that phototropic curvature proceeded as normal in the ubiquitin mutant lines (Fig. 2.2). This finding indicates that phyA ubiquitination at lysine 555 and 603 are not necessary for phyA to modulate phototropic curvature in very low and low fluence rate phototropism. 


\section{phy $^{\mathrm{K} 555 \mathrm{R}, \mathrm{K} 603 \mathrm{R}}$ lines display reduced phototropic bending in high}

\section{intensity blue light}

High intensity blue light has been shown in a previous study to attenuate phototropism. We find in high intensity phototropic assays that phototropism is reduced in phy $A^{\mathrm{K} 555 \mathrm{R}, \mathrm{K} 603 \mathrm{R}}$ lines (Fig. 2.4). This finding is interesting in that it has the opposite effect of the absence of phyA. Given the fact that sites that mark phyA for degradation were altered, this result suggests that phyA abundance could be impacting phototropic curvature.

\section{phy $A^{\mathrm{K} 555 \mathrm{R}, \mathrm{K} 603 \mathrm{R}}$ is stabilized in high intensity blue light}

Phototropic data in high intensity blue light suggests that stabilization of phy A by alterations to its $\mathrm{NPH} 3$ and blue light-dependent ubiquitination sites could result in a decrease in phototropic curvature by stabilizing phy $A$ and increasing its

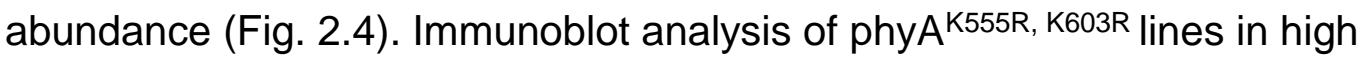
intensity blue light reveals that phyA is partially stabilized (Fig. 2.6 A). This finding in conjunction with high intensity blue light-induced phototropism data indicates that phy ${ }^{\mathrm{K} 555 \mathrm{R}, \mathrm{K} 603 \mathrm{R}}$ stabilization in high intensity blue light results in phototropic reduction under high intensity blue light.

\section{phy ${ }^{\mathrm{K} 555 \mathrm{R}, \mathrm{K} 603 \mathrm{R}}$ ubiquitination levels are not detectably less than in the phyA-GFP transgenic control in immunoblots of immunoprecipitated proteins} Immunoblotting of phy $A^{\mathrm{K} 555 \mathrm{R}, \mathrm{K} 603 \mathrm{R}}$ immunoprecipitated from seedlings treated with blue light does not reveal a distinct difference in phyA ubiquitination levels 
compared to phy-GFP (Fig. 2.6 E). This data suggests that either 1) phyA ubiquitination at other identified lysine residues is masking the detection of differences in ubiquitination levels or 2) that the ubiquitination machinery ubiquitinating phy $A$ is ubiquitinating phy $A$ at other locations. Given the phototropic phenotype in high intensity blue light and phyA stabilization in high intensity blue light, it seems likely that decreases in ubiquitination in phyA are being hidden by the ubiquitination of other phyA sites.

phy $^{\mathrm{K} 555 \mathrm{R}, \mathrm{K} 603 \mathrm{R}}$ degradation is not controlled by phot1, phot2, or NPH3 under high intensity blue light conditions Immunoblot assays of phy $\mathrm{A}^{\mathrm{K} 555 \mathrm{R}, \mathrm{K} 603 \mathrm{R}}$ in photoreceptor null mutant backgrounds indicates that phot1, phot2, and NPH3 are not required for high intensity blue light-induced phyA degradation (Fig. 2.8). Given the fact that phyA is able to perceive blue light it is likely that phyA is regulating its own degradation in conditions of high intensity blue light.

\section{Glycine max and Glycine soja display widely different growth responses when grown in far-red light}

While characterizing early light responses in G. max and G. soja we revealed that under conditions of far-red light $G$. soja does not have a dramatic far-red light stimulated response but rather growing in respect to stem elongation like seedlings grown in darkness (Fig. 3.5). This lack of responsiveness in G. max progenitor is interesting and somewhat unexpected. $A$. thaliana seedlings grown in a similar light conditions would exhibit strong growth inhibition relative to darkgrown seedlings. 


\section{Glycine max and Glycine soja display a generally similar pattern}

\section{of growth in white, blue, and red light}

Glycine max and Glycine soja display similar modes of development in white, blue, and red light, where growth is inhibited in the hypocotyl and stimulated in the first internode (Figs. 3.2-4). This finding implies that the domestication of $G$. max has not resulted in a drastic alteration in developmental responses in these light conditions

\section{Glycine max produces a second internode more frequently in all}

\section{light conditions than Glycine soja}

Seedlings of G. max produce a second internode in all light conditions with $50 \%$ or greater seedlings displaying some development of a second internode. G. soja in comparison to G. max produces a second internode in white light, but scarcely produces a second internode in blue and red, while never producing a second internode in far-red light (Fig. 3.6). These data suggest that soja does not direct the growth of a second internode unless it perceives the full range of visible light present in white light while G. max produces a second internode with stimulation from any quality of light tested.

\section{Glycine max produces robust root growth in all light conditions and in darkness while G. soja produces root growth when stimulated by light}

G. max domestication has resulted in seedlings with robust root development in all light conditions and when grown in darkness (Fig. 3.7). In contrast, G. soja 
roots are stimulated in white light while stimulated to a lesser extent in monochromatic light conditions. When looking at the average length of roots in each light condition, G. max exhibits roots nearly as long in darkness as in light conditions while G. soja produces short roots in darkness and long roots when treated with light, most notably in white light. These data taken together likely suggest that G. max is developing long roots in darkness while G. soja does not develop roots until light is perceived. 


\section{LITERATURE CITED:}

Aggarwal, C., Banaś, A. K., Kasprowicz-Maluśki, A., Borghetti, C., Łabuz, J., Dobrucki, J., \& Gabryś, H. (2014). Blue-light-activated phototropin2 trafficking from the cytoplasm to Golgi/post-Golgi vesicles. Journal of Experimental Botany, 65(12), 3263-3276. https://doi.org/10.1093/jxb/eru172

Ahmad, M., \& Cashmore, A. R. (1993). HY4 gene of A. thaliana encodes a protein with characteristics of a blue-light photoreceptor. Nature, 366(6451), 162-166. https://doi.org/10.1038/366162a0

Ahmad, M., Jarillo, J. A., Cashmore, A. R., Ahmad, M., Jarillo, J. A., \& Cashmore, A. R. (1998). Chimeric Proteins between cry1 and cry2 Arabidopsis Blue Light Photoreceptors Indicate Overlapping Functions and Varying Protein Stability Published by : American Society of Plant Biologists ( ASPB ) Linked references are available on JSTOR for this artic, 10(2), 197207.

Askinosie, S., \& Liscum, E. (2016). Blue light- and ubiquitin-dependent influence on phototropin 1 abundance and movement at the plasma membrane .

Babourina, O., Godfrey, L., \& Voltchanskii, K. (2004). Changes in ion fluxes during phototropic bending of etiolated oat coleoptiles. Annals of Botany, 94(1), 187-194. https://doi.org/10.1093/aob/mch126

Bartolini, F., \& Gundersen, G. G. (2006). Generation of noncentrosomal microtubule arrays. Journal of Cell Science, 119(20), 4155-4163. https://doi.org/10.1242/jcs.03227

Baskin, T. I. (2001). On the alignment of cellulose microfibrils by cortical 
microtubules: A review and a model. Protoplasma, 215(1-4), 150-171. https://doi.org/10.1007/BF01280311

Baum, G., Long, J. C., Jenkins, G. I., \& Trewavas, a J. (1999). Stimulation of the blue light phototropic receptor NPH1 causes a transient increase in cytosolic Ca2+. Proceedings of the National Academy of Sciences of the United States of America, 96(23), 13554-13559. https://doi.org/10.1073/pnas.96.23.13554

Baxter, I. (2003). Genomic Comparison of P-Type ATPase Ion Pumps in Arabidopsis and Rice. Plant Physiology, 132(2), 618-628. https://doi.org/10.1104/pp.103.021923

Benjamins, R. (2003). PINOID-Mediated Signaling Involves Calcium-Binding Proteins. Plant Physiology, 132(3), 1623-1630.

https://doi.org/10.1104/pp.103.019943

Bennett, M. J., Marchant, A., Green, H. G., May, S. T., Sally, P., Millner, P. A., ... Feldmann, K. A. (1996). Arabidopsis AUX1 Gene : A Permease-Like Regulator of Root Gravitropism Published by : American Association for the Advancement of Science Stable URL : http://www.jstor.org/stable/2891528 JSTOR is a not-for-profit service that helps scholars, researchers, 273(5277), 948-950.

Bennett, S. R. M., Alvarez, J., Bossinger, G., \& Smyth, D. R. (1995). Morphogenesis in pinoid mutants of Arabidopsis thaliana. The Plant Journal, 8(4), 505-520. https://doi.org/10.1046/j.1365-313X.1995.8040505.x Bennett, T. (2015). PIN proteins and the evolution of plant development. Trends 
in Plant Science, 20(8), 498-507.

https://doi.org/10.1016/j.tplants.2015.05.005

Berben, G., Legrain, M., Gilliquet, V., \& Hilger, F. (1990). The yeast regulatory gene PHO4 encodes a helix-loop-helix motif. Yeast.

https://doi.org/10.1002/yea.320060510

Blakeslee, J. J., Bandyopadhyay, A., Peer, W. A., Makam, S. N., \& Murphy, A. S. (2004). Relocalization of the PIN1 auxin efflux facilitator plays a role in phototropic responses. Plant Physiology, 134(1), 28-31.

https://doi.org/10.1104/pp.103.031690

Boer, D. R., Freire-Rios, A., Van Den Berg, W. A. M., Saaki, T., Manfield, I. W., Kepinski, S., ... Coll, M. (2014). Structural basis for DNA binding specificity by the auxin-dependent ARF transcription factors. Cell, 156(3), 577-589. https://doi.org/10.1016/j.cell.201312.027

Bögre, L., Ökrész, L., Henriques, R., \& Anthony, R. G. (2003). Growth signalling pathways in Arabidopsis and the AGC protein kinases. Trends in Plant Science, 8(9), 424-431. https://doi.org/10.1016/S1360-1385(03)00188-2

Boylan, M. T., \& Quail, P. H. (1991). Phytochrome a overexpression inhibits hypocotyl elongation in transgenic Arabidopsis. Proceedings of the National Academy of Sciences of the United States of America, 88(23), 1080610810. https://doi.org/10.1073/pnas.88.23.10806

Briggs, W. R. (2001). Photoreceptors in Plant Photomorphogenesis to Date. Five Phytochromes, Two Cryptochromes, One Phototropin, and One Superchrome. Plant Physiology, 125(1), 85-88. 
https://doi.org/10.1104/pp.125.1.85

Briggs, W. R., \& Huala, E. (1999). Blue-Light Photoreceptors Inhigher Plants. Annu. Rev. Cell Dev. Biol, 62, 33-62.

Carretero-Paulet, L., Galstyan, A., Roig-Villanova, I., Martinez-Garcia, J. F., Bilbao-Castro, J. R., \& Robertson, D. L. (2010). Genome-Wide Classification and Evolutionary Analysis of the bHLH Family of Transcription Factors in Arabidopsis, Poplar, Rice, Moss, and Algae. Plant Physiology, 153(3), 13981412. https://doi.org/10.1104/pp.110.153593

Casal, J. J. (2000). Phytochromes, Cryptochromes, Phototropin: Photoreceptor Interactions in Plants. Photochemistry and Photobiology, 71(1), 1. https://doi.org/10.1562/0031-8655(2000)071<0001:PCPPII>2.0.CO;2

Casal, J. J. (2013). Photoreceptor Signaling Networks in Plant Responses to Shade. Annual Review of Plant Biology, 64(1), 403-427. https://doi.org/10.1146/annurev-arplant-050312-120221

Casal, J. J., \& Qüesta, J. I. (2018). Light and temperature cues: multitasking receptors and transcriptional integrators. New Phytologist, 217(3), 10291034. https://doi.org/10.1111/nph.14890

Celaya, R. B., \& Liscum, E. (2007). Phototropins and Associated Signaling: Providing the Power of Movement in Higher Plants $\uparrow$. Photochemistry and Photobiology, 81(1), 73-80. https://doi.org/10.1111/j.17511097.2005.tb01524.x

Chacón-Sánchez, M. I., \& Martínez-Castillo, J. (2017). Testing Domestication Scenarios of Lima Bean (Phaseolus lunatus L.) in Mesoamerica: Insights 
from Genome-Wide Genetic Markers. Frontiers in Plant Science, 8(September), 1-20. https://doi.org/10.3389/fpls.2017.01551

Chae, K., Isaacs, C. G., Reeves, P. H., Maloney, G. S., Muday, G. K., Nagpal, P., \& Reed, J. W. (2012). Arabidopsis SMALL AUXIN UP RNA63 promotes hypocotyl and stamen filament elongation. Plant Journal, 71(4), 684-697. https://doi.org/10.1111/j.1365-313X.2012.05024.x

Chan, W. M. (2006). Ubiquitination of p53 at Multiple Sites in the DNA-Binding Domain. Molecular Cancer Research, 4(1), 15-25. https://doi.org/10.1158/1541-7786.MCR-05-0097

Chaves, I., Pokorny, R., Byrdin, M., Hoang, N., Ritz, T., Brettel, K., ... Ahmad, M. (2011). The Cryptochromes: Blue Light Photoreceptors in Plants and Animals. Annual Review of Plant Biology, 62(1), 335-364. https://doi.org/10.1146/annurev-arplant-042110-103759 Chen, H., Huang, X., Gusmaroli, G., Terzaghi, W., Lau, O. S., Yanagawa, Y., ... Deng, X. W. (2010). Arabidopsis CULLIN4-Damaged DNA Binding Protein 1 Interacts with CONSTITUTIVELY PHOTOMORPHOGENIC1SUPPRESSOR OF PHYA Complexes to Regulate Photomorphogenesis and Flowering Time. The Plant Cell, 22(1), 108-123.

https://doi.org/10.1105/tpc.109.065490

Chen, M., \& Chory, J. (2011). Phytochrome signaling mechanisms and the control of plant development. Trends in Cell Biology, 21(11), 664-671. https://doi.org/10.1016/j.tcb.2011.07.002.Phytochrome Cheng, Y., Qin, G., Dai, X., \& Zhao, Y. (2007). NPY1, a BTB-NPH3-like protein, 
plays a critical role in auxin-regulated organogenesis in Arabidopsis.

Proceedings of the National Academy of Sciences, 104(47), 18825-18829. https://doi.org/10.1073/pnas.0708506104

Cheng, Y., Qin, G., Dai, X., \& Zhao, Y. (2008). NPY genes and AGC kinases define two key steps in auxin-mediated organogenesis in Arabidopsis. Proceedings of the National Academy of Sciences of the United States of America, 105(52), 21017-21022. https://doi.org/10.1073/pnas.0809761106 Cho, M., Lee, S. H., \& Cho, H.-T. (2007). P-Glycoprotein4 Displays Auxin Efflux Transporter-Like Action in Arabidopsis Root Hair Cells and Tobacco Cells. The Plant Cell Online, 19(12), 3930-3943. https://doi.org/10.1105/tpc.107.054288

Christensen, S. K., Dagenais, N., Chory, J., \& Weigel, D. (2000). Regulation of Auxin Response by the Protein Kinase PINOID. Cell, 100(4), 469-478. https://doi.org/10.1016/S0092-8674(00)80682-0

Christie, J. M., Blackwood, L., Petersen, J., \& Sullivan, S. (2015). Plant flavoprotein photoreceptors. Plant and Cell Physiology, 56(3), 401-413. https://doi.org/10.1093/pcp/pcu196

Christie, J. M., Reymond, P., Powell, G. K., Bernasconi, P., Raibekas, A. A., Liscum, E., \& Briggs, W. R. (1998). Arabidopsis NPH1: A flavoprotein with the properties of a photoreceptor for phototropism. Science, 282(5394), 1698-1701. https://doi.org/10.1126/science.282.5394.1698

Christie, J. M., Salomon, M., Nozue, K., Wada, M., \& Briggs, W. R. (1999). LOV (light, oxygen, or voltage) domains of the blue-light photoreceptor 
phototropin (nph1): Binding sites for the chromophore flavin mononucleotide. Proceedings of the National Academy of Sciences, 96(15), 8779-8783. https://doi.org/10.1073/pnas.96.15.8779

Christie, J. M., Yang, H., Richter, G. L., Sullivan, S., Thomson, C. E., Lin, J., ... Murphy, A. S. (2011). Phot1 inhibition of ABCB19 primes lateral Auxin Fluxes in the Shoot Apex required for Phototropism. PLoS Biology, 9(6). https://doi.org/10.1371/journal.pbio.1001076

Christie, J., Suetsugu, N., Sullivan, S., \& Wada, M. (2017). Shining Light on the Function of NPH3/RPT2-like Proteins in Phototropin Signalling. Plant Physiology, 176(February), pp.00835.2017. https://doi.org/10.1104/pp.17.00835

Ciarbelli, A. R., Ciolfi, A., Salvucci, S., Ruzza, V., Possenti, M., Carabelli, M., ... Ruberti, I. (2008). The Arabidopsis Homeodomain-leucine Zipper II gene family: Diversity and redundancy. Plant Molecular Biology, 68(4-5), 465478. https://doi.org/10.1007/s11103-008-9383-8

Clack, T., Mathews, S., \& Sharrock, R. A. (1994). The phytochrome apoprotein family in Arabidopsis is encoded by five genes: the sequences and expression of PHYD and PHYE. Plant Molecular Biology, 25(3), 413-427. https://doi.org/10.1007/BF00043870

Cockram, J., Jones, H., Leigh, F. J., O'Sullivan, D., Powell, W., Laurie, D. A., \& Greenland, A. J. (2007). Control of flowering time in temperate cereals: Genes, domestication, and sustainable productivity. Journal of Experimental Botany, 58(6), 1231-1244. https://doi.org/10.1093/jxb/erm042 
Cosgrove, D. J. (1997). Relaxation in a High-Stress Environment: The Molecular Bases of Extensible Cell Walls and Cell Enlargement. The Plant Cell Online, 9(7), 1031-1041. https://doi.org/10.1105/tpc.9.7.1031

Cosgrove, D. J. (2000). Loosening of plant cell walls by expansins. Nature, 407(6802), 321-326. https://doi.org/10.1038/35030000

Crane, M., \& Boga, D. (2017). A commentary: Rethinking approaches to resilience and mental health training. Journal of Military and Veterans' Health, 25(1), 30-33. https://doi.org/10.1002/ejoc.201200111

Crosson, S., Rajagopal, S., \& Moffat, K. (2003). The LOV domain family:

Photoresponsive signaling modules coupled to diverse output domains. Biochemistry, 42(1), 2-10. https://doi.org/10.1021/bi026978| de Carbonnel, M., Davis, P., Roelfsema, M. R. G., Inoue, S. -i., Schepens, I., Lariguet, P., ... Fankhauser, C. (2010). The Arabidopsis PHYTOCHROME KINASE SUBSTRATE2 Protein Is a Phototropin Signaling Element That Regulates Leaf Flattening and Leaf Positioning. Plant Physiology, 152(3), 1391-1405. https://doi.org/10.1104/pp.109.150441

DeBlasio, S. L., Mullen, J. L., Luesse, D. R., \& Hangarter, R. P. (2003).

Phytochrome modulation of blue light-induced chloroplast movements in Arabidopsis. Plant Physiology, 133(4), 1471-1479.

https://doi.org/10.1104/pp.103.029116

Demarsy, E., Schepens, I., Okajima, K., Hersch, M., Bergmann, S., Christie, J., ... Fankhauser, C. (2012). Phytochrome Kinase Substrate 4 is phosphorylated by the phototropin 1 photoreceptor. EMBO Journal, 31(16), 
3457-3467. https://doi.org/10.1038/emboj.2012.186

Deshaies, R. J., \& Joazeiro, C. A. P. (2009). RING Domain E3 Ubiquitin Ligases. Annual Review of Biochemistry, 78(1), 399-434. https://doi.org/10.1146/annurev.biochem.78.101807.093809

Dezfulian, M. H., Jalili, E., Roberto, D. K. A., Moss, B. L., Khoo, K., Nemhauser, J. L., \& Crosby, W. L. (2016). Oligomerization of SCFTIR1 Is Essential for Aux/IAA Degradation and Auxin Signaling in Arabidopsis. PLoS Genetics, 12(9), 1-18. https://doi.org/10.1371/journal.pgen.1006301

Ding, Z., GGalván-Ampudia, C. S., Demarsy, E., Łangowski, Ł., Kleine-Vehn, J., Fan, Y., ... Friml, J. (2011). Light-mediated polarization of the PIN3 auxin transporter for the phototropic response in Arabidopsis. Nature Cell Biology, 13(4), 447-453. https://doi.org/10.1038/ncb2208

Doherty, G. J., \& McMahon, H. T. (2009). Mechanisms of Endocytosis. Annual Review of Biochemistry, 78(1), 857-902. https://doi.org/10.1146/annurev.biochem.78.081307.110540

Dümmer, M., Michalski, C., Essen, L. O., Rath, M., Galland, P., \& Forreiter, C. (2016). EHB1 and AGD12, two calcium-dependent proteins affect gravitropism antagonistically in Arabidopsis thaliana. Journal of Plant Physiology, 206, 114-124. https://doi.org/10.1016/j.jplph.2016.09.006

Eckardt, N. a. (2001). From Darkness into Light: Factors Controlling Photomorphogenesis. The Plant Cell, 13(2), 219-221. https://doi.org/10.1105/tpc.13.2.219 Emily L. Stowe-Evans, Darron R. Luesse, and E. L., Stowe-Evans, E. L., 
Luesse, D. R., \& Liscum, E. (2001). The Enhancement of PhototropinInduced Phototropic Curvature in Arabidopsis Occurs via a Photoreversible Phytochrome A-Dependent Modulation of Auxin Responsiveness. Plant Physiology, 126(2), 826-834. https://doi.org/10.1104/pp.126.2.826

Esmon, C. A., Tinsley, A. G., Ljung, K., Sandberg, G., Hearne, L. B., \& Liscum, E. (2006). A gradient of auxin and auxin-dependent transcription precedes tropic growth responses. Proceedings of the National Academy of Sciences of the United States of America, 103(1), 236-241.

https://doi.org/10.1073/pnas.0507127103

Fankhauser, C. (2001). The Phytochromes, a Family of Red/Far-red Absorbing Photoreceptors. Journal of Biological Chemistry, 276(15), 11453-11456. https://doi.org/10.1074/jbc.R100006200

Fankhauser, C., \& Christie, J. M. (2015). Plant phototropic growth. Current Biology, 25(9), R384-R389. https://doi.org/10.1016/j.cub.2015.03.020

Fankhauser, C., Yeh, K. C., Lagarias, J. C., Zhang, H., Elich, T. D., \& Chory, J. (1999). PKS1, a substrate phosphorylated by phytochrome that modulates light signaling in Arabidopsis. Science, 284(5419), 1539-1541.

https://doi.org/10.1126/science.284.5419.1539

Felle, H. (1988). Auxin causes oscillations of cytosolic free calcium and $\mathrm{pH}$ in\&lt;i\&gt;Zea mays\&lt;/i\&gt; coleoptiles. Planta. https://doi.org/10.1007/bf00634478

Figueroa, P., Gusmaroli, G., Serino, G., Habashi, J., Ma, L., Shen, Y., ... Deng, X. W. (2005). Arabidopsis has two redundant Cullin3 proteins that are 
essential for embryo development and that interact with RBX1 and BTB proteins to form multisubunit E3 ubiquitin ligase complexes in vivo. The Plant Cell, 17(4), 1180-1195. https://doi.org/10.1105/tpc.105.031989

Fiorucci, A. S., \& Fankhauser, C. (2017). Plant Strategies for Enhancing Access to Sunlight. Current Biology, 27(17), R931-R940.

https://doi.org/10.1016/j.cub.2017.05.085

Folta, K. M., Lieg, E. J., Durham, T., \& Spalding, E. P. (2003). Primary Inhibition of Hypocotyl Growth and Phototropisrn Depend Differently o ..., 133(December), 1464-1470. https://doi.org/10.1104/pp.103.024372.ways

Franklin, K. A., Larner, V. S., \& Whitelam, C. C. (2005). The signal transducing photoreceptors of plants. International Journal of Developmental Biology, 49(5-6), 653-664. https://doi.org/10.1387/ijdb.051989kf

Franklin, K. A., \& Quail, P. H. (2010). Phytochrome functions in Arabidopsis development. Journal of Experimental Botany, 61(1), 11-24. https://doi.org/10.1093/jxb/erp304

Franklin, K. A., \& Whitelam, G. C. (2005). Phytochromes and shade-avoidance responses in plants. Annals of Botany, 96(2), 169-175.

https://doi.org/10.1093/aob/mci165

Friml, J. (2003). Auxin transport $Đ$ shaping the plant Prelude $Đ$ The linden tree of innocence. Current Opinion in Plant Biology, 6(6), 7-12. https://doi.org/10.1016/S1369-5266(02)00003-1

Friml, J., Wiśniewska, J., Benková, E., Mendgen, K., \& Palme, K. (2002). Lateral relocation of auxin efflux regulator PIN3 mediates tropism in Arabidopsis. 
Nature, 415(6873), 806-809. https://doi.org/10.1038/415806a

Friml, J., Yang, X., Michniewicz, M., Weijers, D., Quint, A., Tietz, O., ... Offringa, R. (2004). A PINOID-dependent binary switch in apical-basal PIN polar targeting directs auxin efflux. Science, 306(5697), 862-865. https://doi.org/10.1126/science.1100618

Furutani, M., Kajiwara, T., Kato, T., Treml, B. S., Stockum, C., Torres-Ruiz, R. A., \& Tasaka, M. (2007). The gene MACCHI-BOU 4/ENHANCER OF PINOID encodes a NPH3-like protein and reveals similarities between organogenesis and phototropism at the molecular level. Development, 134(21), 3849-3859. https://doi.org/10.1242/dev.009654

Galen, C., Huddle, J., \& Liscum, E. (2004). An experimental test of the adaptive evolution of phototropins: Blue-light photoreceptors controlling phototropism in Arabidopsis thaliana. Evolution, 58(3), 515-523.

https://doi.org/10.1111/j.0014-3820.2004.tb01675.x

Galen, C., Rabenold, J. J., \& Liscum, E. (2007). Functional ecology of a blue light photoreceptor: Effects of phototropin-1 on root growth enhance drought tolerance in Arabidopsis thaliana. New Phytologist, 173(1), 91-99. https://doi.org/10.1111/j.1469-8137.2006.01893.x

Gehring, C., Williams, D., Cody, S., \& Parish, R. (1990). Phototropism and geotropism in maize coleoptiles are spatially correlated with increases in cytosolic free calcium. Nature, 345, 528-30.

https://doi.org/10.1038/345528a0

Geldner, N. (2003). Partial loss-of-function alleles reveal a role for GNOM in 
auxin transport-related, post-embryonic development of Arabidopsis.

Development, 131(2), 389-400. https://doi.org/10.1242/dev.00926

Geldner, N., Anders, N., Wolters, H., Keicher, J., Kornberger, W., Muller, P., ...

Jürgens, G. (2003). The Arabidopsis GNOM ARF-GEF mediates endosomal recycling, auxin transport, and auxin-dependent plant growth. Cell, 112(2), 219-230. https://doi.org/10.1016/S0092-8674(03)00003-5

Genschik, P., Sumara, I., \& Lechner, E. (2013). The emerging family of CULLIN3-RING ubiquitin ligases (CRL3s): Cellular functions and disease implications. EMBO Journal, 32(17), 2307-2320.

https://doi.org/10.1038/emboj.2013.173

Georg H.H. Borner, Kathryn S. Lilley, Timothy J. Stevens, and P. D., \& Borner, G. H. H. (2003). Identification of Glycosylphosphatidylinositol-Anchored Proteins in Arabidopsis. A Proteomic and Genomic Analysis. Plant Physiology, 132(2), 568-577. https://doi.org/10.1104/pp.103.021170

Gladman, N. P., Marshall, R. S., Lee, K.-H., \& Vierstra, R. D. (2016). The Proteasome Stress Regulon Is Controlled by a Pair of NAC Transcription Factors in Arabidopsis. The Plant Cell, 28(6), 1279-1296.

https://doi.org/10.1105/tpc.15.01022

Grones, P., \& Friml, J. (2015). Auxin transporters and binding proteins at a glance. Journal of Cell Science, 128(1), 1-7.

https://doi.org/10.1242/jcs.159418

Grunewald, W., \& Friml, J. (2010). The march of the PINs: Developmental plasticity by dynamic polar targeting in plant cells. EMBO Journal, 29(16), 
2700-2714. https://doi.org/10.1038/emboj.2010.181

Guilfoyle, T. J. (2015). The PB1 Domain in Auxin Response Factor and Aux/IAA Proteins: A Versatile Protein Interaction Module in the Auxin Response. The Plant Cell Online, 27(1), 33-43. https://doi.org/10.1105/tpc.114.132753

Guo, J., Wang, Y., Song, C., Zhou, J., Qiu, L., Huang, H., \& Wang, Y. (2010). A single origin and moderate bottleneck during domestication of soybean (Glycine max): Implications from microsatellites and nucleotide sequences. Annals of Botany, 106(3), 505-514. https://doi.org/10.1093/aob/mcq125

Ha, C. M., Jun, J. H., \& Fletcher, J. C. (2010). Shoot apical meristem form and function. Current Topics in Developmental Biology, 91(C), 103-140. https://doi.org/10.1016/S0070-2153(10)91004-1

Haga, K. (2005). The Rice COLEOPTILE PHOTOTROPISM1 Gene Encoding an Ortholog of Arabidopsis NPH3 Is Required for Phototropism of Coleoptiles and Lateral Translocation of Auxin. The Plant Cell Online, 17(1), 103-115. https://doi.org/10.1105/tpc.104.028357

Haga, K., Tsuchida-Mayama, T., Yamada, M., \& Sakai, T. (2015). Arabidopsis ROOT PHOTOTROPISM2 Contributes to the Adaptation to High-Intensity Light in Phototropic Responses. The Plant Cell, 27(4), 1098-1112. https://doi.org/10.1105/tpc.15.00178

Hagen, G., \& Guilfoyle, T. (2002). Auxin-responsive gene expression: Genes, promoters and regulatory factors. Plant Molecular Biology, 49(3-4), 373385. https://doi.org/10.1023/A:1015207114117

Haglund, K., \& Dikic, I. (2005). Ubiquitylation and cell signaling. EMBO Journal, 
24(19), 3353-3359. https://doi.org/10.1038/sj.emboj.7600808

Han, I.-S., Tseng, T.-S., Eisinger, W., \& Briggs, W. R. (2008). Phytochrome A Regulates the Intracellular Distribution of Phototropin 1-Green Fluorescent Protein in Arabidopsis thaliana. The Plant Cell Online, 20(10), 2835-2847. https://doi.org/10.1105/tpc.108.059915

Han, I. S., Cho, H. Y., Moni, A., Lee, A. Y., \& Briggs, W. R. (2013). Investigations on the photoregulation of chloroplast movement and leaf positioning in arabidopsis. Plant and Cell Physiology, 54(1), 48-56.

https://doi.org/10.1093/pcp/pcs098

Han, M., Park, Y., Kim, I., Kim, E.-H., Yu, T.-K., Rhee, S., \& Suh, J.-Y. (2014).

Structural basis for the auxin-induced transcriptional regulation by Aux/IAA17. Proceedings of the National Academy of Sciences, 111(52), 18613-18618. https://doi.org/10.1073/pnas.1419525112

Harada, A., Sakai, T., \& Okada, K. (2003). phot1 and phot2 mediate blue lightinduced transient increases in cytosolic $\mathrm{Ca} 2+$ differently in Arabidopsis leaves. Proceedings of the National Academy of Sciences, 100(14), 85838588. https://doi.org/10.1073/pnas. 1336802100

Harada, A., \& Shimazaki, K. (2007). Phototropins and Blue Light-dependent Calcium Signaling in Higher Plants†. Photochemistry and Photobiology, 83(1), 102-111. https://doi.org/10.1562/2006-03-08-IR-837

Harada, A., Takemiya, A., Inoue, S. I., Sakai, T., \& Shimazaki, K. I. (2013). Role of RPT2 in leaf positioning and flattening and a possible inhibition of phot2 signaling by phot1. Plant and Cell Physiology, 54(1), 36-47. 
https://doi.org/10.1093/pcp/pcs094

Harper, R. M. R. M., Stowe-Evans, E. L., Luesse, D. R., Muto, H., Tatematsu, K., Watahiki, M. K., ... Briggs, W. R. (2001). The Enhancement of PhototropinInduced Phototropic Curvature in Arabidopsis Occurs via a Photoreversible Phytochrome A-Dependent Modulation of Auxin Responsiveness. The Plant Cell, 282(1), 993-997. https://doi.org/10.1104/pp.103.030601.far-red Harper, R. M., Stowe-Evans, E. L., Luesse, D. R., Muto, H., Tatematsu, K., Watahiki, M. K., ... Liscum, E. (2000). The NPH4 locus encodes the auxin response factor ARF7, a conditional regulator of differential growth in aerial Arabidopsis tissue. The Plant Cell, 12(5), 757-70.

https://doi.org/10.1105/tpc.12.5.757

Harper, S. M., Christie, J. M., \& Gardner, K. H. (2004). Disruption of the LOV-Ja helix interaction activates phototropin kinase activity. Biochemistry, 43(51), 16184-16192. https://doi.org/10.1021/bi048092i

Hartman, G. L., West, E. D., \& Herman, T. K. (2011). Crops that feed the World 2. Soybean-worldwide production, use, and constraints caused by pathogens and pests. Food Security, 3(1), 5-17. https://doi.org/10.1007/s12571-010-0108-x Haruta, M., Burch, H. L., Nelson, R. B., Barrett-Wilt, G., Kline, K. G., Mohsin, S. B., ... Sussman, M. R. (2010). Molecular characterization of mutant Arabidopsis plants with reduced plasma membrane proton pump activity. Journal of Biological Chemistry, 285(23), 17918-17929. https://doi.org/10.1074/jbc.M110.101733 
Hennig, L., Poppe, C., Unger, S., \& Schäfer, E. (1999). Control of hypocotyl elongation in Arabidopsis thaliana by photoreceptor interaction. Planta, 208(2), 257-263. https://doi.org/10.1007/s004250050557

Hohm, T., Preuten, T., \& Fankhauser, C. (2013). Phototropism: Translating light into directional growth. American Journal of Botany, 100(1), 47-59. https://doi.org/10.3732/ajb.1200299

Hotton, S. K., \& Callis, J. (2008). Regulation of Cullin RING Ligases. Annual Review of Plant Biology, 59(1), 467-489. https://doi.org/10.1146/annurev.arplant.58.032806.104011

Huala, E., Oeller, P. W., Liscum, E., Han, I. S., Larsen, E., \& Briggs, W. R. (1997). Arabidopsis NPH1: A protein kinase with a putative redox-sensing domain. Science, 278(5346), 2120-2123.

https://doi.org/10.1126/science.278.5346.2120

Huang, F., Kemel Zago, M., Abas, L., van Marion, A., Galván-Ampudia, C. S., \& Offringa, R. (2010). Phosphorylation of Conserved PIN Motifs Directs Arabidopsis PIN1 Polarity and Auxin Transport. The Plant Cell, 22(4), 11291142. https://doi.org/10.1105/tpc. 109.072678

Hughes, J. (2013). Phytochrome Cytoplasmic Signaling. Annual Review of Plant Biology, 64(1), 377-402. https://doi.org/10.1146/annurev-arplant-050312120045

Hyten, D. L., Song, Q., Zhu, Y., Choi, I.-Y., Nelson, R. L., Costa, J. M., ... Cregan, P. B. (2006). Impacts of genetic bottlenecks on soybean genome diversity. Proceedings of the National Academy of Sciences, 103(45), 
16666-16671. https://doi.org/10.1073/pnas.0604379103

lino, M. (2006). Toward understanding the ecological functions of tropisms: Interactions among and effects of light on tropisms. Current Opinion in Plant Biology, 9(1), 89-93. https://doi.org/10.1016/j.pbi.2005.11.012

Inada, S., Ohgishi, M., Mayama, T., Okada, K., \& Sakai, T. (2004). RPT2 is a signal transducer involved in phototropic response and stomatal opening by association with phototropin 1 in Arabidopsis thaliana. The Plant Cell, 16(4), 887-896. https://doi.org/10.1105/tpc.019901

Inoue, S. -i., Kinoshita, T., Matsumoto, M., Nakayama, K. I., Doi, M., \& Shimazaki, K. -i. (2008). Blue light-induced autophosphorylation of phototropin is a primary step for signaling. Proceedings of the National Academy of Sciences, 105(14), 5626-5631. https://doi.org/10.1073/pnas.0709189105 Inoue, S. -i., Matsushita, T., Tomokiyo, Y., Matsumoto, M., Nakayama, K. I., Kinoshita, T., \& Shimazaki, K. -i. (2011). Functional Analyses of the Activation Loop of Phototropin2 in Arabidopsis. Plant Physiology, 156(1), 117-128. https://doi.org/10.1104/pp.111.175943

Inoue, S. I., Kinoshita, T., Takemiya, A., Doi, M., \& Shimazaki, K. I. (2008). Leaf positioning of Arabidopsis in response to blue light. Molecular Plant, 1(1), 15-26. https://doi.org/10.1093/mp/ssm001 Jabben, M., Shanklin, J., \& Vierstras, D. (1989). Ubiquitin-Phytochrome Conjugates, 264(9), 4998-5005.

Jaedicke, K., Lichtenthäler, A. L., Meyberg, R., Zeidler, M., \& Hughes, J. (2012). 
A phytochrome-phototropin light signaling complex at the plasma membrane. Proceedings of the National Academy of Sciences, 109(30), 12231-12236. https://doi.org/10.1073/pnas.1120203109

Jain, M., Tyagi, A. K., \& Khurana, J. P. (2006). Genome-wide analysis, evolutionary expansion, and expression of early auxin-responsive SAUR gene family in rice (Oryza sativa). Genomics, 88(3), 360-371. https://doi.org/10.1016/j.ygeno.2006.04.008

Janoudi, A., \& Poff, K. L. (1993). Desensitization and Recovery of Phototropic Re s p o n s i ve n e ss i n Arabidopsis thaliana. Plant Physiol., (101), 11751180.

Janoudi, a K., Gordon, W. R., Wagner, D., Quail, P., \& Poff, K. L. (1997). Multiple phytochromes are involved in red-light-induced enhancement of first-positive phototropism in Arabidopsis thaliana. Plant Physiology, 113(3), 975-979. https://doi.org/10.1104/pp.113.3.975

Janoudi A-K, Konjevic, R., Apel, P., \& Poff, K. L. (1992). Time threshold for second positive phototropism is decreased by a preirradiation with red light. Plant Physiology, 99(4), 1422-1425. https://doi.org/10.1104/pp.99.4.1422 Jarillo, J. A., Gabrys, H., Capel, J., Alonso, J. M., Ecker, J. R., \& Cashmore, A. R. (2001). Phototropin-related NPL1 controls chloroplast relocation induced by blue light. Nature, 410(6831), 952-954. https://doi.org/10.1038/35073622 Jenkins, G. I. (2014). Structure and function of the UV-B photoreceptor UVR8. Current Opinion in Structural Biology, 29(January), 52-57. https://doi.org/10.1016/j.sbi.2014.09.004 
Jones, M. A., Feeney, K. A., Kelly, S. M., \& Christie, J. M. (2007). Mutational analysis of phototropin 1 provides insights into the mechanism underlying LOV2 signal transmission. Journal of Biological Chemistry, 282(9), 64056414. https://doi.org/10.1074/jbc.M605969200

Josse, E. M., \& Halliday, K. J. (2008). Skotomorphogenesis: The Dark Side of Light Signalling. Current Biology, 18(24), R1144-R1146. https://doi.org/10.1016/j.cub.2008.10.034

Kagawa, T., Sakai, T., Suetsugu, N., Oikawa, K., Ishiguro, S., Kato, T., ... Wada, M. (2001). Arabidopsis NPL1: A phototropin homolog controlling the chloroplast high-light avoidance response. Science, 291(5511), 2138-2141. https://doi.org/10.1126/science.291.5511.2138

Kaiserli, E., Sullivan, S., Jones, M. A., Feeney, K. A., \& Christie, J. M. (2009). Domain Swapping to Assess the Mechanistic Basis of Arabidopsis Phototropin 1 Receptor Kinase Activation and Endocytosis by Blue Light. The Plant Cell, 21(10), 3226-3244. https://doi.org/10.1105/tpc.109.067876 Kami, C., Allenbach, L., Zourelidou, M., Ljung, K., Schütz, F., Isono, E., ... Fankhauser, C. (2014). Reduced phototropism in pks mutants may be due to altered auxin-regulated gene expression or reduced lateral auxin transport. Plant Journal, 77(3), 393-403. https://doi.org/10.1111/tpj.12395

Kami, C., Hersch, M., Trevisan, M., Genoud, T., Hiltbrunner, A., Bergmann, S., \& Fankhauser, C. (2012). Nuclear Phytochrome A Signaling Promotes Phototropism in Arabidopsis. The Plant Cell Online, 24(2), 566-576. https://doi.org/10.1105/tpc.111.095083 
Kami, C., Lorrain, S., Hornitschek, P., \& Fankhauser, C. (2010). Light-regulated plant growth and development. Current Topics in Developmental Biology, 91(C), 29-66. https://doi.org/10.1016/S0070-2153(10)91002-8

Kansup, J., Tsugama, D., Liu, S., \& Takano, T. (2014). Arabidopsis G-protein $\beta$ subunit AGB1 interacts with NPH3 and is involved in phototropism. Biochemical and Biophysical Research Communications, 445(1), 54-57. https://doi.org/10.1016/j.bbrc.2014.01.106

Khurana, J. P., \& Poff, K. L. (1989). Mutants of Arabidopsis thaliana with altered phototropisme. Planta.

Kim, J., Harter, K., \& Theologis, A. (1997). Protein-protein interactions among the Aux/IAA proteins. Proceedings of the National Academy of Sciences of the United States of America, 94(22), 11786-91.

https://doi.org/10.1073/pnas.94.22.11786

Kim, J., Shen, Y., Han, Y., Park, J., Kirchenbauer, D., Soh, M., ... Scha, E. (2004). Phytochrome Phosphorylation Modulates Light Signaling by In uencing the Protein-Protein Interaction. October, 16(October), 2629-2640. https://doi.org/10.1105/tpc.104.023879.1

Kim, M. Y., Van, K., Kang, Y. J., Kim, K. H., \& Lee, S.-H. (2012). Tracing soybean domestication history: From nucleotide to genome. Breeding Science, 61(5), 445-452. https://doi.org/10.1270/jsbbs.61.445

Kinoshita, T., Doi, M., \& Suetsugu, N. (2001). Regulation of Stomatal Opening. Nature, 414(December), 0-4.

Knauer, T., Dummer, M., Landgraf, F., \& Forreiter, C. (2011). A Negative Effector 
of Blue Light-Induced and Gravitropic Bending in Arabidopsis. Plant Physiology, 156(1), 439-447. https://doi.org/10.1104/pp.110.167411

Kneissl, J., Shinomura, T., Furuya, M., \& Bolle, C. (2008). A rice phytochrome a in Arabidopsis: The role of the $\mathrm{N}$-terminus under red and far-red light. Molecular Plant, 1(1), 84-102. https://doi.org/10.1093/mp/ssm010

Kong, S. G., Kagawa, T., Wada, M., \& Nagatani, A. (2013). A C-terminal membrane association domain of phototropin 2 is necessary for chloroplast movement. Plant and Cell Physiology, 54(1), 57-68.

https://doi.org/10.1093/pcp/pcs132

Kong, S. G., Kinoshita, T., Shimazaki, K. I., Mochizuki, N., Suzuki, T., \& Nagatani, A. (2007). The C-terminal kinase fragment of Arabidopsis phototropin 2 triggers constitutive phototropin responses. Plant Journal, 51(5), 862-873. https://doi.org/10.1111/j.1365-313X.2007.03187.x

Kong, S. G., Suetsugu, N., Kikuchi, S., Nakai, M., Nagatani, A., \& Wada, M. (2013). Both phototropin 1 and 2 localize on the chloroplast outer membrane with distinct localization activity. Plant and Cell Physiology, 54(1), 80-92. https://doi.org/10.1093/pcp/pcs151

Kong, S. G., Suzuki, T., Tamura, K., Mochizuki, N., Hara-Nishimura, I., \& Nagatani, A. (2006). Blue light-induced association of phototropin 2 with the Golgi apparatus. Plant Journal, 45(6), 994-1005. https://doi.org/10.1111/j.1365-313X.2006.02667.x

Kong, S. G., \& Wada, M. (2016). Molecular basis of chloroplast photorelocation movement. Journal of Plant Research, 129(2), 159-166. 
https://doi.org/10.1007/s10265-016-0788-1

Koornneef, M., Rolff, E., \& Spruit, C. J. P. J. P. (1980). Genetic control of lightinhibited hypocotyl elongation in Arabidopsis thaliana (L.) heynh. Zeitschrift Für Pflanzenphysiologie, 100(2), 147-160. https://doi.org/10.1016/s0044$328 x(80) 80208-x$

Korasick, D. A., Westfall, C. S., Lee, S. G., Nanao, M. H., Dumas, R., Hagen, G., ... Strader, L. C. (2014). Molecular basis for AUXIN RESPONSE FACTOR protein interaction and the control of auxin response repression. Proceedings of the National Academy of Sciences, 111(14), 5427-5432. https://doi.org/10.1073/pnas.1400074111

Kozuka, T., Suetsugu, N., Wada, M., \& Nagatani, A. (2013). Antagonistic regulation of leaf flattening by phytochrome $B$ and phototropin in Arabidopsis thaliana. Plant and Cell Physiology, 54(1), 69-79. https://doi.org/10.1093/pcp/pcs134

Kutschera, U., \& Niklas, K. J. (2009). Evolutionary plant physiology: Charles Darwin's forgotten synthesis. Naturwissenschaften, 96(11), 1339-1354. https://doi.org/10.1007/s00114-009-0604-z

Lalanne, E., Michaelidis, C., Moore, J. M., Gagliano, W., Johnson, A., Patel, R., ... Twell, D. (2004). Analysis of transposon insertion mutants highlights the diversity of mechanisms underlying male progamic development in Arabidopsis. Genetics, 167(4), 1975-1986. https://doi.org/10.1534/genetics.104.030270 Lariguet, P., Boccalandro, H. E., Alonso, J. M., Ecker, J. R., Chory, J., Casal, J. 
J., \& Fankhauser, C. (2003). A Growth Regulatory Loop That Provides Homeostasis to Phytochrome A Signaling. The Plant Cell, 15(December), 2966-2978. https://doi.org/10.1105/tpc.014563.2

Lariguet, P., \& Dunand, C. (2005). Plant photoreceptors: Phylogenetic overview. Journal of Molecular Evolution, 61(4), 559-569.

https://doi.org/10.1007/s00239-004-0294-2

Lariguet, P., \& Fankhauser, C. (2004). Hypocotyl growth orientation in blue light is determined by phytochrome A inhibition of gravitropism and phototropin promotion of phototropism. Plant Journal, 40(5), 826-834.

https://doi.org/10.1111/j.1365-313X.2004.02256.x

Lariguet, P., Schepens, I., Hodgson, D., Pedmale, U. V., Trevisan, M., Kami, C., ... Liscum, E. (2006). PHYTOCHROME KINASE SUBSTRATE 1 is a phototropin 1 binding protein required for phototropism. Proceedings of the National Academy of Sciences, 103(26), 10134-10139.

https://doi.org/10.1073/pnas.0603799103

Lascève, G., Leymarie, J., Olney, M. A., Liscum, E., Christie, J. M., Vavasseur, A., \& Briggs, W. R. (1999). Arabidopsis contains at least four independent blue-light-activated signal transduction pathways. Plant Physiology, 120(2), 605-614. https://doi.org/10.1104/pp.120.2.605

Lau, O. S., \& Deng, X. W. (2012). The photomorphogenic repressors COP1 and DET1: 20 years later. Trends in Plant Science, 17(10), 584-593. https://doi.org/10.1016/j.tplants.2012.05.004

Lavy, M., \& Estelle, M. (2016). Mechanisms of auxin signaling. Development, 
143(18), 3226-3229. https://doi.org/10.1242/dev.131870

Lee, D. H. (1998). Proteasome inhibitors: Valuable new tools for cell biologists. Trends in Cell Biology, 8(10), 397-403. https://doi.org/10.1016/S09628924(98)01346-4

Lee, H. J., Park, Y. J., Ha, J. H., Baldwin, I. T., \& Park, C. M. (2017). Multiple Routes of Light Signaling during Root Photomorphogenesis. Trends in Plant Science, 22(9), 803-812. https://doi.org/10.1016/j.tplants.2017.06.009

Lee, S. S., Lee, S. S., Yang, K. Y., Kim, Y. M., Park, S. Y., Kim, S. Y., \& Soh, M. S. (2006). Overexpression of PRE1 and its homologous genes activates gibberellin-dependent responses in Arabidopsis thaliana. Plant and Cell Physiology, 47(5), 591-600. https://doi.org/10.1093/pcp/pcj026

Lewis, D. R., Miller, N. D., Splitt, B. L., Wu, G., \& Spalding, E. P. (2007). Separating the Roles of Acropetal and Basipetal Auxin Transport on Gravitropism with Mutations in Two Arabidopsis Multidrug Resistance-Like ABC Transporter Genes. The Plant Cell, 19(6), 1838-1850. https://doi.org/10.1105/tpc.107.051599

Li, A. C., \& Cosgrove, D. J. (2001). Grass group I pollen allergens (??-expansins) lack proteinase activity and do not cause wall loosening via proteolysis. European Journal of Biochemistry, 268(15), 4217-4226. https://doi.org/10.1046/j.1432-1327.2001.02336.x

Li, F.-W., Rothfels, C. J., Melkonian, M., Villarreal, J. C., Stevenson, D. W., Graham, S. W., ... Pryer, K. M. (2015). The origin and evolution of phototropins. Frontiers in Plant Science, 6(August), 1-11. 
https://doi.org/10.3389/fpls.2015.00637

Li, X., Duan, X., Jiang, H., Sun, Y., Tang, Y., Yuan, Z., \& Guo, J. (2006).

Genome-Wide Analysis of Basic / Helix-Loop-Helix Transcription Factor

Family in Rice and Arabidopsis. Plant Physiology, 141(August), 1167-1184. https://doi.org/10.1104/pp.106.080580.2001

Li, Y., Dai, X., Cheng, Y., \& Zhao, Y. (2011). NPY genes play an essential role in root gravitropic responses in Arabidopsis. Molecular Plant, 4(1), 171-179. https://doi.org/10.1093/mp/ssq052

Li, Y., Darley, C. P., Ongaro, V., Fleming, A., Schipper, O., Baldauf, S. L., \& McQueen-Mason, S. J. (2002). Plant Expansins Are a Complex Multigene Family with an Ancient Evolutionary Origin. Plant Physiology, 128(3), 854864. https://doi.org/10.1104/pp.010658

Li, Y. H., Li, W., Zhang, C., Yang, L., Chang, R. Z., Gaut, B. S., \& Qiu, L. J. (2010). Genetic diversity in domesticated soybean (Glycine max) and its wild progenitor (Glycine soja) for simple sequence repeat and single-nucleotide polymorphism loci. New Phytologist, 188(1), 242-253.

https://doi.org/10.1111/j.1469-8137.2010.03344.x

Lin, C., Yang, H., Guo, H., Mockler, T., Chen, J., \& Cashmore, A. R. (1998).

Enhancement of blue-light sensitivity of Arabidopsis seedlings by a blue light receptor cryptochrome 2. Proceedings of the National Academy of Sciences, 95(5), 2686-2690. https://doi.org/10.1073/pnas.95.5.2686

Lindeboom, J. J., Nakamura, M., Hibbel, A., Shundyak, K., Gutierrez, R., Ketelaar, T., ... Ehrhardt, D. W. (2013). A mechanism for reorientation of 
cortical microtubule arrays driven by microtubule severing. Science, 342(6163), 1245533-1245533. https://doi.org/10.1126/science.1245533 Liscum, E. (1995). Mutations in the NPH1 Locus of Arabidopsis Disrupt the Perception of Phototropic Stimuli. The Plant Cell Online, 7(4), 473-485. https://doi.org/10.1105/tpc.7.4.473

Liscum, E. (2016). Blue Light-Induced Intracellular Movement of Phototropins: Functional Relevance or Red Herring? Frontiers in Plant Science, 7(June), 1-5. https://doi.org/10.3389/fpls.2016.00827

Liscum, E., Askinosie, S. K., Leuchtman, D. L., Morrow, J., Willenburg, K. T., \& Coats, D. R. (2014). Phototropism: Growing towards an Understanding of Plant Movement. The Plant Cell, 26(1), 38-55. https://doi.org/10.1105/tpc.113.119727

Liscum, E., \& Briggs, W. R. (1996). Mutations of Arabidopsis in potential transduction and response components of the phototropic signaling pathway. Plant Physiology, 112(1), 291-296. https://doi.org/10.1104/pp.112.1.291

Liscum, E., \& Reed, J. W. (2002). Genetics of Aux/IAA and ARF action in plant growth and development. Plant Molecular Biology, 49(3-4), 387-400. https://doi.org/10.1023/A:1015255030047

Liu, B., Yang, Z., Gomez, A., Liu, B., Lin, C., \& Oka, Y. (2016). Signaling mechanisms of plant cryptochromes in Arabidopsis thaliana. Journal of Plant Research, 129(2), 137-148. https://doi.org/10.1007/s10265-015-0782-z Liu, B., Zuo, Z., Liu, H., Liu, X., \& Lin, C. (2011). Arabidopsis cryptochrome 1 
interacts with SPA1 to suppress COP1 activity in response to blue light. Genes and Development, 25(10), 1029-1034.

https://doi.org/10.1101/gad.2025011

Liu, H., Liu, B., Zhao, C., Pepper, M., \& Lin, C. (2012). The action mechanisms of plant cryptochromes. Trends Plant Sci., 16(12), 684-691.

https://doi.org/10.1016/j.tplants.2011.09.002.The

Ludwig, S. R., Habera, L. F., Dellaporta, S. L., \& Wessler, S. R. (1989). Lc, a member of the maize $\mathrm{R}$ gene family responsible for tissue-specific anthocyanin production, encodes a protein similar to transcriptional activators and contains the myc-homology region. Proceedings of the National Academy of Sciences of the United States of America, 86(18), 7092-7096. https://doi.org/10.1073/pnas.86.18.7092

Mao, K., Dong, Q., Li, C., Liu, C., \& Ma, F. (2017). Genome Wide Identification and Characterization of Apple bHLH Transcription Factors and Expression Analysis in Response to Drought and Salt Stress. Frontiers in Plant Science, 8(April), 480. https://doi.org/10.3389/fpls.2017.00480

Mara, C. D., Huang, T., \& Irish, V. F. (2010). The Arabidopsis Floral Homeotic Proteins APETALA3 and PISTILLATA Negatively Regulate the BANQUO Genes Implicated in Light Signaling. The Plant Cell, 22(3), 690-702. https://doi.org/10.1105/tpc.109.065946

Marchler-Bauer, A., Zheng, C., Chitsaz, F., Derbyshire, M. K., Geer, L. Y., Geer, R. C., ... Bryant, S. H. (2013). CDD: Conserved domains and protein threedimensional structure. Nucleic Acids Research, 41(D1), 348-352. 
https://doi.org/10.1093/nar/gks1243

Mawphlang, O. I. L., \& Kharshiing, E. V. (2017). Photoreceptor Mediated Plant Growth Responses: Implications for Photoreceptor Engineering toward Improved Performance in Crops. Frontiers in Plant Science, 8(July), 1-14. https://doi.org/10.3389/fpls.2017.01181

McClure, B. A., \& Guilfoyle, T. (1987). Characterization of a class of small auxininducible soybean polyadenylated RNAs. Plant Molecular Biology. https://doi.org/10.1007/BF00020537

Mcqueen-mason, S., Durachko, D. M., \& Cosgrove, D. J. (1992). Two Endogenous Pmteins That Induce Cell Wall Extension in Plants. Society, 4(November), 1425-1433. https://doi.org/10.1105/tpc.4.11.1425

McSteen, P. (2010). Auxin and monocot development. Cold Spring Harbor Perspectives in Biology, 2(3). https://doi.org/10.1101/cshperspect.a001479 Michalski, C., Dümmer, M., Galland, P., \& Forreiter, C. (2017). Impact of EHB1 and AGD12 on Root and Hypocotyl Phototropism in Arabidopsis thaliana. Journal of Plant Growth Regulation, 36(3), 660-668. https://doi.org/10.1007/s00344-017-9667-9

Møller, S. G., Ingles, P. J., \& Whitelam, G. C. (2002). The Cell Biology of phytochrome signaling. New Phytologist, 154(3), 553-590. https://doi.org/10.1046/j.1469-8137.2002.00419.x

Motchoulski, A., \& Liscum, E. (1999). Arabidopsis NPH3: A NPH1 photoreceptorinteracting protein essential for phototropism. Science, 286(5441), 961-964. https://doi.org/10.1126/science.286.5441.961 
Murre, C., McCaw, P. S., \& Baltimore, D. (1989). A new DNA binding and dimerization motif in immunoglobulin enhancer binding, daughterless, MyoD, and myc proteins. Cell, 56(5), 777-783. https://doi.org/10.1016/00928674(89)90682-X

Nagashima, A., Suzuki, G., Uehara, Y., Saji, K., Furukawa, T., Koshiba, T., ... Sakai, T. (2008). Phytochromes and cryptochromes regulate the differential growth of Arabidopsis hypocotyls in both a PGP19-dependent and a PGP19independent manner. Plant Journal, 53(3), 516-529.

https://doi.org/10.1111/j.1365-313X.2007.03358.x

Nakasako, M., Zikihara, K., Matsuoka, D., Katsura, H., \& Tokutomi, S. (2008). Structural Basis of the LOV1 Dimerization of Arabidopsis Phototropins 1 and 2. Journal of Molecular Biology, 381(3), 718-733.

https://doi.org/10.1016/j.jmb.2008.06.033

Nakazawa, M., Yabe, N., Ichikawa, T., Yamamoto, Y. Y., Yoshizumi, T., Hasunuma, K., \& Matsui, M. (2001). DFL1, an auxin-responsive GH3 gene homologue, negatively regulates shoot cell elongation and lateral root formation, and positively regulates the light response of hypocotyl length. Plant Journal, 25(2), 213-221. https://doi.org/10.1046/j.1365313X.2001.00957.x

Nemhauser, J., \& Chory, J. (2002). Photomorphogenesis. The Arabidopsis Book, 1, e0054. https://doi.org/10.1199/tab.0054

Ni, M., Tepperman, J. M., \& Quail, P. H. (1998). PIF3, a phytochrome-interacting factor necessary for normal photoinduced signal transduction, is a novel 
basic helix-loop-helix protein. Cell, 95(5), 657-667.

https://doi.org/10.1016/S0092-8674(00)81636-0

Nick, P., Bergfeld, R., Schäfer, E., \& Schopfer, P. (1990). Unilateral reorientation of microtubules at the outer epidermal wall during photo- and gravitropic curvature of maize coleoptiles and sunflower hypocotyls. Planta, 181(2), 162-168. https://doi.org/10.1007/BF02411533

Noh, B., Bandyopadhyay, A., Peer, W. A., Spalding, E. P., \& Murphy, A. S. (2003). Enhanced gravi-and photropism in plant mislocalizing the auxin efflux protein PIN1. Nature, 423(June), 999-1002. https://doi.org/10.1038/nature01696.1.

Nozue, K., Kanegae, T., Imaizumi, T., Fukuda, S., Okamoto, H., Yeh, K. C., ... Wada, M. (1998). A phytochrome from the fern Adiantum with features of the putative photoreceptor NPH1. Proceedings of the National Academy of Sciences of the United States of America, 95(26), 15826-15830. https://doi.org/10.1073/pnas.95.26.15826

Ohgishi, M., Saji, K., Okada, K., \& Sakai, T. (2004). Functional analysis of each blue light receptor, cry1, cry2, phot1, and phot2, by using combinatorial multiple mutants in Arabidopsis. Proceedings of the National Academy of Sciences, 101(8), 2223-2228. https://doi.org/10.1073/pnas.0305984101

Okada, K. (1991). Requirement of the Auxin Polar Transport System in Early Stages of Arabidopsis Floral Bud Formation. The Plant Cell Online, 3(7), 677-684. https://doi.org/10.1105/tpc.3.7.677

Okadaa, K., \& Shimuraab, Y. (1992). Mutational Analysis of Root Gravitropism 
and Phototropism of Arabidopsis thaliana Seedlings. Aust. J. Plant Physiol, 19, 439-48. https://doi.org/10.1071/PP9920439

Okushima, Y., Overvoorde, P. J., Arima, K., Alonso, J. M., Chan, A., Chang, C., ... Smith, A. (2005). Functional Genomic Analysis of the AUXIN RESPONSE FACTOR Gene Family Members in Arabidopsis thaliana. The Plant Cell, 17(February), 444-463. https://doi.org/10.1105/tpc.104.028316.2

Paredez, A. R., Somerville, C. R., \& Ehrhardt, D. W. (2006). Visualization of cellulose synthase demonstrates functional association with microtubules. Science, 312(5779), 1491-1495. https://doi.org/10.1126/science.1126551

Park, J. E., Seo, P. J., Lee, A. K., Jung, J. H., Kim, Y. S., \& Park, C. M. (2007). An Arabidopsis GH3 gene, encoding an auxin-conjugating enzyme, mediates phytochrome B-regulated light signals in hypocotyl growth. Plant and Cell Physiology, 48(8), 1236-1241. https://doi.org/10.1093/pcp/pcm086

Park, Y. B., \& Cosgrove, D. J. (2012). A Revised Architecture of Primary Cell Walls Based on Biomechanical Changes Induced by Substrate-Specific Endoglucanases. Plant Physiology, 158(4), 1933-1943. https://doi.org/10.1104/pp.111.192880

Parks, B. M., Quail, P. H., \& Hangarter, R. P. (1996). Phytochrome A regulates red-light induction of phototropic enhancement in Arabidopsis. Plant Physiology, 110(1), 155-162. https://doi.org/10.1104/pp.110.1.155

Parry, G., Delbarre, A., Marchant, A., Swarup, R., Napier, R., PerrotRechenmann, C., \& Bennett, M. J. (2001). Novel auxin transport inhibitors phenocopy the auxin influx carrier mutation aux1. Plant Journal, 25(4), 399- 
406. https://doi.org/10.1046/j.1365-313X.2001.00970.x

Pedmale, U. V., Celaya, R. B., \& Liscum, E. (2002). Phototropism, Mechanisms and Outcomes. The Arabidopsis Book, 8(8), DOI 10.1199/tab.0042 http://www.aspb.org/publicati. https://doi.org/10.1199/tab.0042

Pedmale, U. V., \& Liscum, E. (2007). Regulation of phototropic signaling in Arabidopsis via phosphorylation state changes in the phototropin 1interacting protein NPH3. Journal of Biological Chemistry, 282(27), 1999220001. https://doi.org/10.1074/jbc.M702551200

Peer, W. A., Blakeslee, J. J., Yang, H., \& Murphy, A. S. (2011). Seven things we think we know about auxin transport. Molecular Plant, 4(3), 487-504. https://doi.org/10.1093/mp/ssr034

Petricka, J. J., Clay, N. K., \& Nelson, T. M. (2008). Vein patterning screens and the defectively organized tributaries mutants in Arabidopsis thaliana. Plant Journal, 56(2), 251-263. https://doi.org/10.1111/j.1365-313X.2008.03595.x

Petroutsos, D., Tokutsu, R., Maruyama, S., Flori, S., Greiner, A., Magneschi, L., ... Minagawa, J. (2016). A blue-light photoreceptor mediates the feedback regulation of photosynthesis. Nature, 537(7621), 563-566. https://doi.org/10.1038/nature19358

Pires, N., \& Dolan, L. (2010). Origin and diversification of basic-helix-loop-helix proteins in plants. Molecular Biology and Evolution, 27(4), 862-874. https://doi.org/10.1093/molbev/msp288

Preuten, T., Blackwood, L., Christie, J. M., \& Fankhauser, C. (2015). Lipid anchoring of arabidopsis phototropin 1 to assess the functional significance 
of receptor internalization: Should I stay or should I go? New Phytologist, 206(3), 1038-1050. https://doi.org/10.1111/nph.13299

Pudasaini, A., \& Zoltowski, B. D. (2013). Zeitlupe senses blue-light fluence to mediate circadian timing in arabidopsis thaliana. Biochemistry, 52(40), 7150-7158. https://doi.org/10.1021/bi401027n

Qiu, T., Chen, Y., Li, M., Kong, Y., Zhu, Y., Han, N., .. Wang, J. (2013). The tissue-specific and developmentally regulated expression patterns of the SAUR41 subfamily of SMALL AUXIN UP RNA genes: Potential implications. Plant Signaling and Behavior, 8(8), 15-17. https://doi.org/10.4161/psb.25283

Quint, M., Ito, H., Zhang, W., \& Gray, W. M. (2005). Characterization of a novel temperature-sensitive allele of the CUL1/AXR6 subunit of SCF ubiquitinligases. Plant Journal, 43(3), 371-383. https://doi.org/10.1111/j.1365313X.2005.02449.x

Rademacher, E. H., \& Offringa, R. (2012). Evolutionary Adaptations of Plant AGC Kinases: From Light Signaling to Cell Polarity Regulation. Frontiers in Plant Science, 3(November), 1-16. https://doi.org/10.3389/fpls.2012.00250

Rayle, D. L., \& Cleland, R. (1970). Enhancement of wall loosening and elongation by Acid solutions. Plant Physiology, 46(2), 250-253. https://doi.org/10.1104/pp.46.2.250

Rayle, D. L., \& Cleland, R. E. (1992). The Acid Growth Theory of auxin-induced cell elongation is alive and well. Plant Physiology, 99(4), 1271-1274. https://doi.org/10.1104/pp.99.4.1271

Reed, J. W., Nagatani, A., Elich, T. D., Fagan, M., \& Chory, J. (1994). 
Phytochrome A and Phytochrome B Have Overlapping but Distinct Functions in Arabidopsis Development. Plant Physiology, 104(4), 11391149. https://doi.org/104/4/1139 [pii]

Ren, H., \& Gray, W. M. (2015). SAUR Proteins as Effectors of Hormonal and Environmental Signals in Plant Growth. Molecular Plant, 8(8), 1153-1164. https://doi.org/10.1016/j.molp.2015.05.003

Roberts, D., Pedmale, U. V., Morrow, J., Sachdev, S., Lechner, E., Tang, X., ... Liscum, E. (2011). Modulation of Phototropic Responsiveness in Arabidopsis through Ubiquitination of Phototropin 1 by the CUL3-Ring E3 Ubiquitin Ligase CRL3NPH3. The Plant Cell, 23(10), 3627-3640. https://doi.org/10.1105/tpc.111.087999

Rockwell, N. C. (2006). Phytochome Structure and Signaling Mechanisms. NIH Public Access, 57(26), 837-858. https://doi.org/10.1146/annurev.arplant.56.032604.144208.PHYTOCHOME Rodriguez, L., Gonzalez-Guzman, M., Diaz, M., Rodrigues, A., Izquierdo-Garcia, A. C., Peirats-Llobet, M., ... Rodriguez, P. L. (2014). C2-Domain Abscisic Acid-Related Proteins Mediate the Interaction of PYR/PYL/RCAR Abscisic Acid Receptors with the Plasma Membrane and Regulate Abscisic Acid Sensitivity in Arabidopsis. The Plant Cell Online, 26(12), 4802-4820. https://doi.org/10.1105/tpc.114.129973

Rojas-Pirela, M., Rigden, D. J., Michels, P. A., Cáceres, A. J., Concepción, J. L., \& Quiñones, W. (2018). Structure and function of Per-ARNT-Sim domains and their possible role in the life-cycle biology of Trypanosoma cruzi. 
Molecular and Biochemical Parasitology, 219(April 2017), 52-66.

https://doi.org/10.1016/j.molbiopara.2017.11.002

Rosler, J., Klein, I., \& Zeidler, M. (2007). Arabidopsis fhl/fhy1 double mutant reveals a distinct cytoplasmic action of phytochrome A. Proceedings of the National Academy of Sciences, 104(25), 10737-10742.

https://doi.org/10.1073/pnas.0703855104

Rsler, J., Jaedicke, K., \& Zeidler, M. (2010). Cytoplasmic phytochrome action. Plant and Cell Physiology, 51(8), 1248-1254. https://doi.org/10.1093/pcp/pcq091

Sakai, T., Kagawa, T., Kasahara, M., Swartz, T. E., Christie, J. M., Briggs, W. R., ... Okada, K. (2001). Arabidopsis nph1 and npl1: Blue light receptors that mediate both phototropism and chloroplast relocation. Proceedings of the National Academy of Sciences, 98(12), 6969-6974.

https://doi.org/10.1073/pnas.101137598

Sakai, T., Wada, T., Ishiguro, S., \& Okada, K. (2000). RPT2. A signal transducer of the phototropic response in Arabidopsis. Plant Cell, 12(2), 225-236. https://doi.org/10.1016/S1369-5266(00)80033-3

Sakamoto, K. (2002). Cellular and Subcellular Localization of Phototropin 1. The Plant Cell Online, 14(8), 1723-1735. https://doi.org/10.1105/tpc.003293

Salomon, M., Christie, J. M., Knieb, E., Lempert, U., \& Briggs, W. R. (2000). Photochemical and mutational analysis of the FMN-binding domains of the plant blue light receptor, phototropin. Biochemistry, 39(31), 9401-9410. https://doi.org/10.1021/bi000585+ 
Salomon, M., Lempert, U., \& Rüdiger, W. (2004). Dimerization of the plant photoreceptor phototropin is probably mediated by the LOV1 domain. FEBS Letters, 572(1-3), 8-10. https://doi.org/10.1016/j.febslet.2004.06.081

Sampedro, J., \& Cosgrove, D. J. (2005). The expansin superfamily. Genome Biology, 6(12), 242. https://doi.org/10.1186/gb-2005-6-12-242

Sancar, A. (2004). Photolyase and cryptochrome blue-light photoreceptors. Advances in Protein Chemistry, 69, 73-100. https://doi.org/10.1016/S00653233(04)69003-6

Sanders, D., Pelloux, J., Brownlee, C., \& Harper, J. F. (2002). Calcium at the Crossroads of Signaling. The Plant Cell, 14(suppl 1), S401-S417. https://doi.org/10.1105/tpc.002899

Santner, A. A., \& Watson, J. C. (2006). The WAG1 and WAG2 protein kinases negatively regulate root waving in Arabidopsis. Plant Journal, 45(5), 752764. https://doi.org/10.1111/j.1365-313X.2005.02641.x

Sawa, S., Ohgishi, M., Goda, H., Higuchi, K., Shimada, Y., Yoshida, S., \& Koshiba, T. (2002). The HAT2 gene, a member of the HD-Zip gene family, isolated as an auxin inducible gene by DNA microarray screening, affects auxin response in Arabidopsis. Plant Journal, 32(6), 1011-1022. https://doi.org/10.1046/j.1365-313X.2002.01488.x

Schepens, I., Boccalandro, H. E., Kami, C., Casal, J. J., \& Fankhauser, C. (2008). PHYTOCHROME KINASE SUBSTRATE4 Modulates PhytochromeMediated Control of Hypocotyl Growth Orientation. Plant Physiology, 147(2), 661-671. https://doi.org/10.1104/pp.108.118166 
Schindelin, J., Arganda-Carreras, I., Frise, E., Kaynig, V., Longair, M., Pietzsch, T., ... A., C. (2012). Fiji: An open source platform for biological image analysis. Nature Methods, 9(7), 676-682. https://doi.org/10.1038/nmeth.2019.Fiji

Schindelin, J., Rueden, C. T., Hiner, M. C., \& Eliceiri, K. W. (2015). The ImageJ ecosystem: An open platform for biomedical image analysis. Molecular Reproduction and Development, 82(7-8), 518-529.

https://doi.org/10.1002/mrd.22489

Sedbrook, J. C., Carroll, K. L., Hung, K. F., Masson, P. H., \& Somerville, C. R. (2002). The Arabidopsis SKU5 Gene Encodes an Extracellular Glycosyl Phosphatidylinositol-Anchored Glycoprotein Involved in Directional Root Growth. The Plant Cell Online, 14(7), 1635-1648.

https://doi.org/10.1105/tpc.002360.1996

Seo, H. S., Watanabe, E., Tokutomi, S., Nagatani, A., \& Chua, N. H. (2004). Photoreceptor ubiquitination by COP1 E3 ligase desensitizes phytochrome A signaling. Genes and Development, 18(6), 617-622. https://doi.org/10.1101/gad.1187804

Serrano-Bueno, G., Romero-Campero, F. J., Lucas-Reina, E., Romero, J. M., \& Valverde, F. (2017). Evolution of photoperiod sensing in plants and algae. Current Opinion in Plant Biology, 37, 10-17. https://doi.org/10.1016/j.pbi.2017.03.007

Shanklin, J., Jabben, M., \& Vierstra, R. D. (1987). Red light-induced formation of ubiquitin-phytochrome conjugates: Identification of possible intermediates of 
phytochrome degradation. Proceedings of the National Academy of

Sciences of the United States of America, 84(2), 359-63.

https://doi.org/10.1073/pnas.84.2.359

Shanklin, J., Jabben, M., \& Vierstra, R. D. (1989). Partial Purification and Peptide Mapping of Ubiquitin-Phytochrome Conjugates from Oat. Biochemistry, 28(14), 6028-6034. https://doi.org/10.1021/bi00440a046

Sheng Yuan, Yajun Wu, and D. J. C. (2001). A Fungal Endoglucanase with Plant Cell Wall Extension Activity. Plant Physiology, 127(1), 324-333. https://doi.org/10.1104/pp.127.1.324

Sherman-Broyles, S., Bombarely, A., Powell, A. F., Doyle, J. L., Egan, A. N., Coate, J. E., \& Doyle, J. J. (2014). The wild side of a major crop: Soybean's perennial cousins from down under. American Journal of Botany, 101(10), 1651-1665. https://doi.org/10.3732/ajb.1400121

SMITH, H., \& WHITELAM, G. C. (1997). The shade avoidance syndrome: multiple responses mediated by multiple phytochromes. Plant, Cell and Environment, 20(6), 840-844. https://doi.org/10.1046/j.1365-3040.1997.d01104.x

Spartz, A. K., Lee, S. H., Wenger, J. P., Gonzalez, N., Inzé, D., Peer, W. A., ... William, M. (2012). The SAUR19 subfamily of SMALL AUXIN UP RNA genes promote cell expansion, 70(6), 978-990.

https://doi.org/10.1111/j.1365-313X.2012.04946.x.The

Spartz, A. K., Lor, V. S., Ren, H., Olszewski, N. E., Miller, N. D., Wu, G., ... Gray, W. M. (2017). Constitutive Expression of Arabidopsis SMALL AUXIN UP 
RNA19 ( SAUR19) in Tomato Confers Auxin-Independent Hypocotyl Elongation. Plant Physiology, 173(2), 1453-1462.

https://doi.org/10.1104/pp.16.01514

Spartz, A. K., Ren, H., Park, M. Y., Grandt, K. N., Lee, S. H., Murphy, A. S., ... Gray, W. M. (2014). SAUR Inhibition of PP2C-D Phosphatases Activates Plasma Membrane H+-ATPases to Promote Cell Expansion in Arabidopsis. The Plant Cell, 26(5), 2129-2142. https://doi.org/10.1105/tpc.114.126037

Staswick, P. E. (2005). Characterization of an Arabidopsis Enzyme Family That Conjugates Amino Acids to Indole-3-Acetic Acid. The Plant Cell Online, 17(2), 616-627. https://doi.org/10.1105/tpc.104.026690

Staswick, P. E., Staswick, P. E., Tiryaki, I., Tiryaki, I., Rowe, M. L., \& Rowe, M. L. (2002). Jasmonate Response Locus. Plant Cell, 14(June), 1405-1415. https://doi.org/10.1105/tpc.000885.defect

Stogios, P. J., Downs, G. S., Jauhal, J. J. S., Nandra, S. K., \& Privé, G. G. (2005). Sequence and structural analysis of BTB domain proteins. Genome Biology, 6(10), R82. https://doi.org/10.1186/gb-2005-6-10-r82

Stone, B. B., Stowe-Evans, E. L., Harper, R. M., Brandon Celaya, R., Ljung, K., Sandberg, G., \& Liscum, E. (2008). Disruptions in AUX1-dependent auxin influx alter hypocotyl phototropism in Arabidopsis. Molecular Plant, 1(1), 129-144. https://doi.org/10.1093/mp/ssm013

Stowe-Evans, E. L., Harper, R. M., Motchoulski, a V, \& Liscum, E. (1998). $\mathrm{NPH} 4$, a conditional modulator of auxin-dependent differential growth responses in Arabidopsis. Plant Physiology, 118(4), 1265-1275. 
https://doi.org/10.1104/pp.118.4.1265

Strader, L. C., \& Zhao, Y. (2016). Auxin perception and downstream events.

Current Opinion in Plant Biology, 33, 8-14.

https://doi.org/10.1016/j.pbi.2016.04.004

Stupar, R. M. (2010). Into the wild: The soybean genome meets its undomesticated relative. Proceedings of the National Academy of Sciences, 107(51), 21947-21948. https://doi.org/10.1073/pnas.1016809108

Suetsugu, N., Mittmann, F., Wagner, G., Hughes, J., \& Wada, M. (2005). A chimeric photoreceptor gene, NEOCHROME, has arisen twice during plant evolution. Proceedings of the National Academy of Sciences of the United States of America, 102(38), 13705-13709.

https://doi.org/10.1073/pnas.0504734102

Suetsugu, N., Takemiya, A., Kong, S.-G., Higa, T., Komatsu, A., Shimazaki, K., ... Wada, M. (2016). RPT2/NCH1 subfamily of NPH3-like proteins is essential for the chloroplast accumulation response in land plants. Proceedings of the National Academy of Sciences, 113(37), 10424-10429. https://doi.org/10.1073/pnas.1602151113

Sullivan, S., Kaiserli, E., Tseng, T. S., \& Christie, J. M. (2010). Subcellular localization and turnover of Arabidopsis phototropin 1. Plant Signaling and Behavior, 5(2), 184-186. https://doi.org/10.4161/psb.5.2.11082

Sullivan, S., Thomson, C. E., Lamont, D. J., Jones, M. A., \& Christie, J. M. (2008). In Vivo Phosphorylation Site Mapping and Functional Characterization of Arabidopsis Phototropin 1. Molecular Plant, 1(1), 178- 
194. https://doi.org/10.1093/mp/ssm017

Takahashi, K., Hayashi, K. -i., \& Kinoshita, T. (2012). Auxin Activates the Plasma Membrane H+-ATPase by Phosphorylation during Hypocotyl Elongation in Arabidopsis. Plant Physiology, 159(2), 632-641.

https://doi.org/10.1104/pp.112.196428

Takayuki Kohchi, Keiko Mukougawa, Nicole Frankenberg, Munehisa Masuda, Akiho Yokota, and J. C. L. (2001). The Arabidopsis HY2 Gene Encodes Phytochromobilin Synthase, a Ferredoxin-Dependent Biliverdin Reductase. The Plant Cell Online, 13(2), 425-436. https://doi.org/10.1105/tpc.13.2.425

Takemiya, A., Inoue, S., \& Doi, M. (2005). Phototropins promote plant growth in response to blue light in low light environments. The Plant Cell ..., 17(April), 1120-1127. https://doi.org/10.1105/tpc.104.030049.2

Takuya Muramoto, Takayuki Kohchi, Akiho Yokota, Inhwan Hwang, and H. M. G. (1999). The Arabidopsis Photomorphogenic Mutant hy1 Is Deficient in Phytochrome Chromophore Biosynthesis as a Result of a Mutation in a Plastid Heme Oxygenase. The Plant Cell Online, 11(3), 335-348. https://doi.org/10.1105/tpc.11.3.335

Tatematsu, K. (2004). MASSUGU2 Encodes Aux/IAA19, an Auxin-Regulated Protein That Functions Together with the Transcriptional Activator NPH4/ARF7 to Regulate Differential Growth Responses of Hypocotyl and Formation of Lateral Roots in Arabidopsis thaliana. The Plant Cell Online, 16(2), 379-393. https://doi.org/10.1105/tpc.018630

Thomann, A., Lechner, E., Hansen, M., Dumbliauskas, E., Parmentier, Y., 
Kieber, J., ... Genschik, P. (2009). Arabidopsis CULLIN3 genes regulate primary root growth and patterning by ethylene-dependent and -independent mechanisms. PLoS Genetics, 5(1).

https://doi.org/10.1371/journal.pgen.1000328

Titapiwatanakun, B., Blakeslee, J. J., Bandyopadhyay, A., Yang, H., Mravec, J., Sauer, M., ... Murphy, A. S. (2009). ABCB19/PGP19 stabilises PIN1 in membrane microdomains in Arabidopsis. Plant Journal, 57(1), 27-44. https://doi.org/10.1111/j.1365-313X.2008.03668.x

Tokutomi, S., Matsuoka, D., \& Zikihara, K. (2008). Molecular structure and regulation of phototropin kinase by blue light. Biochimica et Biophysica Acta - Proteins and Proteomics, 1784(1), 133-142. https://doi.org/10.1016/j.bbapap.2007.09.010

Toledo-Ortiz, G., Kiryu, Y., Kobayashi, J., Oka, Y., Kim, Y., Nam, H. G., ... Nagatani, A. (2010). Subcellular sites of the signal transduction and degradation of phytochrome A. Plant and Cell Physiology, 51(10), 16481660. https://doi.org/10.1093/pcp/pcq121

Toth, J., Ismaiel, A. A., \& Chen, J. S. (1999). The ald gene, encoding a coenzyme A-acylating aldehyde dehydrogenase, distinguishes Clostridium beijerinckii and two other solvent-producing clostridia from Clostridium acetobutylicum. Applied and Environmental Microbiology, 65(11), 49734980. https://doi.org/10.1016/0003-2697(76)90527-3

Treml, B. S. (2005). The gene ENHANCER OF PINOID controls cotyledon development in the Arabidopsis embryo. Development, 132(18), 4063-4074. 
https://doi.org/10.1242/dev.01969

Tseng, T.-S., \& Briggs, W. R. (2010). The Arabidopsis ren1-1 Mutation Impairs Dephosphorylation of Phot2, Resulting in Enhanced Blue Light Responses. The Plant Cell, 22(2), 392-402. https://doi.org/10.1105/tpc.109.066423

Tsuchida-Mayama, T., Nakano, M., Uehara, Y., Sano, M., Fujisawa, N., Okada, K., \& Sakai, T. (2008). Mapping of the phosphorylation sites on the phototropic signal transducer, NPH3. Plant Science, 174(6), 626-633. https://doi.org/10.1016/j.plantsci.2008.03.018

Tsuchida-Mayama, T., Sakai, T., Hanada, A., Uehara, Y., Asami, T., \& Yamaguchi, S. (2010). Role of the phytochrome and cryptochrome signaling pathways in hypocotyl phototropism. Plant Journal, 62(4), 653-662. https://doi.org/10.1111/j.1365-313X.2010.04180.x

Tsz, K. F., Yam, C. H., \& Poon, R. Y. C. (2005). The N-terminal regulatory domain of cyclin A contains redundant ubiquitination targeting sequences and acceptor sites. Cell Cycle, 4(10), 1411-1420. https://doi.org/10.4161/cc.4.10.2046

Ulijasz, A. T., Cornilescu, G., Cornilescu, C. C., Zhang, J., Rivera, M., Markley, J. L., \& Vierstra, R. D. (2010). Structural basis for the photoconversion of a phytochrome to the activated Pfr form. Nature, 463(7278), 250-254. https://doi.org/10.1038/nature08671

Ulmasov, T., Hagen, G., \& Guilfoyle, T. J. (2017). ARF1, a Transcription Factor That Binds to Auxin Response Elements Author ( s ): Tim Ulmasov, Gretchen Hagen and Tom J . Guilfoyle Published by : American Association 
for the Advancement of Science Stable URL :

http://www.jstor.org/stable/2893420 REFERE, 276(5320), 1865-1868.

Urano, D., Chen, J.-G., Botella, J. R., \& Jones, A. M. (2013). Heterotrimeric G protein signalling in the plant kingdom. Open Biology, 3(3), 120186-120186. https://doi.org/10.1098/rsob.120186

Wahid, H., Ahmad, S., Nor, M. A. M., \& Rashid, M. A. (2017). Prestasi kecekapan pengurusan kewangan dan agihan zakat: perbandingan antara majlis agama islam negeri di Malaysia. Jurnal Ekonomi Malaysia, 51(2), 3954. https://doi.org/10.1017/CBO9781107415324.004

Wan, Y., Jasik, J., Wang, L., Hao, H., Volkmann, D., Menzel, D., ... Lin, J. (2012). The Signal Transducer NPH3 Integrates the Phototropin1 Photosensor with PIN2-Based Polar Auxin Transport in Arabidopsis Root Phototropism. The Plant Cell Online, 24(2), 551-565. https://doi.org/10.1105/tpc.111.094284

Wan, Y. L., Eisinger, W., Ehrhardt, D., Kubitscheck, U., Baluska, F., \& Briggs, W. (2008). The subcellular localization and blue-light-induced movement of phototropin 1-GFP in etiolated seedlings of Arabidopsis thaliana. Molecular Plant, 1(1), 103-117. https://doi.org/10.1093/mp/ssm011

Westfall, C. S., Herrmann, J., Chen, Q., Wang, S., \& Jez, J. M. (2010). Modulating plant hormones by enzyme action. Plant Signaling \& Behavior, 5(12), 1607-1612. https://doi.org/10.4161/psb.5.12.13941

Whippo, C. W., \& Hangarter, R. P. (2003). Second Positive Phototropism Results from Coordinated Co-Action of the Phototropins and Cryptochromes1. Plant 
Physiology, 132(3), 1499-1507. https://doi.org/10.1104/pp.102.018481.)

Whippo, C. W., \& Hangarter, R. P. (2004). Phytochrome modulation of blue-lightinduced phototropism. Plant, Cell and Environment, 27(10), 1223-1228. https://doi.org/10.1111/j.1365-3040.2004.01227.x

Whitelam, G. C., Patel, S., \& Devlin, P. F. (1998). Phytochromes and photomorphogenesis in Arabidopsis. Philosophical Transactions of the Royal Society B: Biological Sciences, 353(1374), 1445-1453.

https://doi.org/10.1098/rstb.1998.0300

Willige, B. C., Ahlers, S., Zourelidou, M., Barbosa, I. C. R., Demarsy, E., Trevisan, M., ... Schwechheimer, C. (2013). D6PK AGCVIII Kinases Are Required for Auxin Transport and Phototropic Hypocotyl Bending in Arabidopsis. The Plant Cell, 25(5), 1674-1688.

https://doi.org/10.1105/tpc.113.111484

Wu, J., Liu, S., He, Y., Guan, X., Zhu, X., Cheng, L., ... Lu, G. (2012). Genomewide analysis of SAUR gene family in Solanaceae species. Gene, 509(1), 38-50. https://doi.org/10.1016/j.gene.2012.08.002

Xu, G., \& Jaffrey, S. R. (2011). The new landscape of protein ubiquitination. Nature Biotechnology, 29(12), 1098-1100. https://doi.org/10.1038/nbt.2061

Xu, G., \& Jaffrey, S. R. (2013). Proteomic identification of protein ubiquitination events. Biotechnology and Genetic Engineering Reviews, 29(1), 73-109. https://doi.org/10.1080/02648725.2013.801232

Xu, G., Paige, J. S., \& Jaffrey, S. R. (2010). Global analysis of lysine ubiquitination by ubiquitin remnant immunoaffinity profiling. Nature 
Biotechnology, 28(8), 868-873. https://doi.org/10.1038/nbt.1654

Yennawar, N. H., Li, L.-C., Dudzinski, D. M., Tabuchi, A., \& Cosgrove, D. J. (2006). Crystal structure and activities of EXPB1 (Zea m 1), a beta-expansin and group-1 pollen allergen from maize. Proceedings of the National Academy of Sciences of the United States of America, 103(40), 1466414671. https://doi.org/10.1073/pnas.0605979103

Zandomeni, K., \& Schopfer, P. (1993). Reorientation of microtubules at the outer epidermal wall of maize coleoptiles by phytochrome, blue-light photoreceptor, and auxin. Protoplasma, 173(3-4), 103-112. https://doi.org/10.1007/BF01378999

Zazímalová, E., Murphy, A. S., Yang, H., Hoyerová, K., \& Hosek, P. (2010). Auxin transporters--why so many? Cold Spring Harbor Perspectives in Biology, 2(3), a001552. https://doi.org/10.1101/cshperspect.a001552

Zhang, X. S., \& O'Neill, S. D. (1993). Ovary and Gametophyte Development Are Coordinately Regulated by Auxin and Ethylene following Pollination. The Plant Cell, 5(4), 403-418. https://doi.org/10.1105/tpc.5.4.403

Zhang, L., Du, L., Shen, C., Yang, Y., \& Poovaiah, B. W. (2014). Regulation of plant immunity through ubiquitin-mediated modulation of $\mathrm{Ca} 2+$-calmodulinAtSR1/CAMTA3 signaling. Plant Journal, 78(2), 269-281. https://doi.org/10.1111/tpj.12473

Zhao, X., Wang, Y.-L., Qiao, X.-R., Wang, J., Wang, L.-D., Xu, C.-S., \& Zhang, X. (2013). Phototropins Function in High-Intensity Blue Light-Induced Hypocotyl Phototropism in Arabidopsis by Altering Cytosolic Calcium. Plant Physiology, 
162(3), 1539-1551. https://doi.org/10.1104/pp.113.216556

Zhao, Y. (2010). Auxin biosynthesis and its role in plant development. Annu Rev Plant Biol, 61, 49-64. https://doi.org/10.1146/annurev-arplant-042809112308.Auxin

Zientara-Rytter, K., \& Sirko, A. (2016). To deliver or to degrade - an interplay of the ubiquitin-proteasome system, autophagy and vesicular transport in plants. FEBS Journal, 283, 3534-3555. https://doi.org/10.1111/febs.13712

Zoltowski, B. D., \& Imaizumi, T. (2014). Structure and function of the ZTL/FKF1/LKP2 group proteins in arabidopsis. Enzymes, 35, 213-239. https://doi.org/10.1016/B978-0-12-801922-1.00009-9

Zourelidou, M., Muller, I., Willige, B. C., Nill, C., Jikumaru, Y., Li, H., \& Schwechheimer, C. (2009). The polarly localized D6 PROTEIN KINASE is required for efficient auxin transport in Arabidopsis thaliana. Development, 136(4), 627-636. https://doi.org/10.1242/dev.028365 


\section{VITA}

I was born April $12^{\text {th }}, 1989$, the second of three children to parents with more commitment to love and care for their children than most any child ever hopes for. Throughout the course of my childhood, I had one consistent passion, learn everything I could about anything and everything science. My first word they tell me was bug. I wanted a better microscope for my $10^{\text {th }}$ birthday because I had exhausted the power of the cheap one I had. I made rock and bug collections and dreamed... of being a paleontologist, geologist, entomologist, electrician, engineer, horticulturalist, botanist, microscopy expert—of being a scientist. I could exhaust a hundred pages describing the various experiments I did in the kitchen, ruining my mom's Tupperware.

As I grew older, my passion for science became directed more firmly towards plants. At fifteen, I designed and built my own greenhouse, which I used to house some bonsais in and start vegetables. When I started college I was around sixteen. There was only one thing that I wanted to study--biology. I graduated in 2011 with high honors, made the Dean's list eight times, and won just about every scholarly award my university offered. I knew I was not done, the idea of being "done" with education did not appeal to me, and I applied to graduate schools, accepting an invitation to join the University of MissouriColumbia biology department in 2012.

I hope to apply the knowledge I have to providing skills and helping wherever I find a position. I will take my love for science and continue to learn and to grow as long as I live. 